ALEA, Lat. Am. J. Probab. Math. Stat. 17, 205-252 (2020)

DOI: 10.30757/ALEA.v17-09

\title{
Existence of the anchored isoperimetric profile in supercritical bond percolation in dimension two and higher
}

\author{
Barbara Dembin \\ Université de Paris, \\ 5 rue Thomas Mann, \\ 75013 Paris, France. \\ E-mail address: bdembin@lpsm.paris \\ URL: http://www.lpsm.paris/pageperso/bdembin/home.html
}

\begin{abstract}
Let $d \geq 2$. We consider an i.i.d. supercritical bond percolation on $\mathbb{Z}^{d}$, every edge is open with a probability $p>p_{c}(d)$, where $p_{c}(d)$ denotes the critical point. We condition on the event that 0 belongs to the infinite cluster $\mathcal{C}_{\infty}$ and we consider connected subgraphs of $\mathcal{C}_{\infty}$ having at most $n^{d}$ vertices and containing 0 . Among these subgraphs, we are interested in the ones that minimize the open edge boundary size to volume ratio. These minimizers properly rescaled converge towards a translate of a deterministic shape and their open edge boundary size to volume ratio properly rescaled converges towards a deterministic constant.
\end{abstract}

\section{Introduction}

Isoperimetric problems are among the oldest problems in mathematics. They consist in finding sets that maximize the volume given a constraint on the perimeter or equivalently that minimize the perimeter to volume ratio given a constraint on the volume. These problems can be formulated in the anisotropic case. Given a norm $\nu$ on $\mathbb{R}^{d}$ and $S$ a continuous subset of $\mathbb{R}^{d}$, we define the tension exerted at a point $x$ in the boundary $\partial S$ of $S$ to be $\nu\left(n_{S}(x)\right) n_{S}(x)$, where $n_{S}(x)$ is the exterior unit normal vector of $S$ at $x$. The quantity $\nu\left(n_{S}(x)\right)$ corresponds to the intensity of the tension that is exerted at $x$. We define the surface energy of $S$ as the integral of the intensity of the surface tension $\nu\left(n_{S}(x)\right)$ over the boundary $\partial S$. An anisotropic isoperimetric problem consists in finding sets that minimize the surface energy to volume ratio given a constraint on the volume. To solve this problem, in Wulff (1901), Wulff introduced through the Wulff construction a shape achieving

Received by the editors April 4th, 2019; accepted October 28th, 2019.

2010 Mathematics Subject Classification. 60K35, 82B43.

Key words and phrases. Percolation, anchored isoperimetric profile.

Research supported by the ANR project PPPP (ANR-16-CE40-0016). 
the infimum. This shape is called the Wulff crystal, it corresponds to the unit ball for a norm built upon $\nu$. Later, Taylor proved in Taylor (1975) that this shape properly rescaled is the unique minimizer, up to translations and modifications on a null set, of the associated isoperimetric problem.

The study of isoperimetric problems in the discrete setting is more recent. In the continuous setting, we study the perimeter to volume ratio, in the context of graphs, the analogous problem is the study of the size of edge boundary to volume ratio. This can be encoded by the Cheeger constant. For a finite graph $\mathcal{G}=(V(\mathcal{G}), E(\mathcal{G}))$, we define the edge boundary $\partial_{\mathcal{G}} A$ of a subset $A$ of $V(\mathcal{G})$ as

$$
\partial_{\mathcal{G}} A=\{e=\langle x, y\rangle \in E(\mathcal{G}): x \in A, y \notin A\} .
$$

We denote by $\partial A$ the edge boundary of $A$ in $\left(\mathbb{Z}^{d}, \mathbb{E}^{d}\right)$ and by $|B|$ the cardinal of the finite set $B$. The isoperimetric constant, also called Cheeger constant, is defined as

$$
\varphi_{\mathcal{G}}=\min \left\{\frac{\left|\partial_{\mathcal{G}} A\right|}{|A|}: A \subset V(\mathcal{G}), 0<|A| \leq \frac{|V(\mathcal{G})|}{2}\right\} .
$$

The continuous version of this constant was introduced by Cheeger in his thesis Cheeger (1970) in order to obtain a lower bound for the smallest eigenvalue of the Laplacian. The discrete version of the Cheeger constant was introduced by Alon and Milman (1985) and Gabber and Galil (1981). The isoperimetric constant of a graph gives information on its geometry.

Let $d \geq 2$. We consider an i.i.d. supercritical bond percolation on $\mathbb{Z}^{d}$, every edge is open with a probability $p>p_{c}(d)$, where $p_{c}(d)$ denotes the critical parameter for this percolation. We know that there exists almost surely a unique infinite open cluster $\mathcal{C}_{\infty}$ (Grimmett, 1999). In this paper, we want to study the geometry of $\mathcal{C}_{\infty}$ through its Cheeger constant. However, if we minimize the isoperimetric ratio over all possible subgraphs of $\mathcal{C}_{\infty}$ without any constraint on the size, one can show that $\varphi_{\mathcal{C}_{\infty}}=0$ almost surely. For that reason, we shall minimize the isoperimetric ratio over all possible subgraphs of $\mathcal{C}_{\infty}$ given a constraint on the size. There are several ways to do it. We can for instance study the Cheeger constant of the graph $\mathcal{C}_{n}=\mathcal{C}_{\infty} \cap[-n, n]^{d}$ or of the largest connected component $\widetilde{\mathcal{C}}_{n}$ of $\mathcal{C}_{n}$ for $n \geq 1$. As we have $\varphi_{\mathcal{C}_{\infty}}=0$ almost surely, the isoperimetric constants $\varphi_{\mathcal{C}_{n}}$ and $\varphi_{\widetilde{\mathcal{C}}_{n}}$ go to 0 when $n$ goes to infinity. Benjamini and Mossel (2003), Mathieu and Remy (2004), Fabien Rau (2006), Berger, Biskup, Hoffman and Kozma (Berger et al., 2008), Pete (2008) proved that $\varphi_{\widetilde{\mathcal{C}}_{n}}$ is of order $n^{-1} \dot{\tilde{C}_{n}}$ Roughly speaking, by analogy with the full lattice, we expect that subgraphs of $\widetilde{\mathcal{C}}_{n}$ that minimize the isoperimetic ratio have an edge boundary size of order $n^{d-1}$ and a size of order $n^{d}$, this is coherent with the fact that $\varphi_{\widetilde{\mathcal{C}}_{n}}$ is of order $n^{-1}$. This led Benjamini to conjecture that for $p>p_{c}(d)$, the limit of $n \varphi_{\widetilde{\mathcal{C}}_{n}}$ when $n$ goes to infinity exists and is a positive deterministic constant.

This conjecture was solved in dimension 2 by Biskup, Louidor, Procaccia and Rosenthal in Biskup et al. (2015) and by Gold in dimension 3 in Gold (2018). They worked on a modified Cheeger constant. Instead of considering the open edge boundary of subgraphs within $\mathcal{C}_{n}$, they considered the open edge boundary within the whole infinite cluster $\mathcal{C}_{\infty}$, this is more natural because $\mathcal{C}_{n}$ has been artificially created by restricting $\mathcal{C}_{\infty}$ to the box $[-n, n]^{d}$. They also added a stronger constraint on the size of subgraphs of $\mathcal{C}_{n}$ to ensure that minimizers do not touch the boundary of the box $[-n, n]^{d}$. Moreover, the subgraphs achieving the minimum, properly 
rescaled, converge towards a deterministic shape that is the Wulff crystal. Namely, it is the shape solving the continuous anisotropic isoperimetric problem associated with a norm $\beta_{p}$ corresponding to the surface tension in the percolation setting. The quantity $n \varphi_{\mathcal{C}_{n}}$ converges towards the solution of a continuous isoperimetric problem.

Dealing with the isoperimetric ratio within $\mathcal{C}_{n}$ needs to be done with caution. Indeed, we do not want minimizers to be close to the boundary of $\mathcal{C}_{n}$ because this boundary does not exist in $\mathcal{C}_{\infty}$. There is another way to define the Cheeger constant of $\mathcal{C}_{\infty}$, that is more natural in the sense that we do not restrict minimizers to remain in the box $[-n, n]^{d}$. This is called the anchored isoperimetric profile $\varphi_{n}$ and it is defined by:

$$
\varphi_{n}=\min \left\{\frac{\left|\partial_{\mathcal{C}_{\infty}} H\right|}{|H|}: 0 \in H \subset \mathcal{C}_{\infty}, \text { H connected, } 0<|H| \leq n^{d}\right\},
$$

where we condition on the event $\left\{0 \in \mathcal{C}_{\infty}\right\}$. We say that $H$ is a valid subgraph if $0 \in H \subset \mathcal{C}_{\infty}, H$ is connected and $|H| \leq n^{d}$. We also define

$$
\partial^{o} H=\{e \in \partial H, e \text { is open }\} .
$$

Note that if $H \subset \mathcal{C}_{\infty}$, then $\partial_{\mathcal{C}_{\infty}} H=\partial^{o} H$. For each $n$, let $\mathcal{G}_{n}$ be the set of the valid subgraphs that achieve the infimum in $\varphi_{n}$. In this context, a minimizer $G_{n} \in \mathcal{G}_{n}$ can go potentially very far from 0 . The minimizer $G_{n}$ properly rescaled does not belong anymore to a compact set. This lack of compactness is the main issue to overcome to prove that the limit exists. It was done in dimension 2 in Biskup et al. (2015), with a specific norm that cannot be extended to higher dimensions. We need to introduce some definitions to be able to define properly a limit shape in dimension $d \geq 2$. In order to build a continuous limit shape, we shall define a continuous analogue of the open edge boundary. In fact, we will see that the open edge boundary may be interpreted in term of a surface tension $\mathcal{I}$, in the following sense. Given a norm $\tau$ on $\mathbb{R}^{d}$ and a subset $E$ of $\mathbb{R}^{d}$ having a regular boundary, we define $\mathcal{I}_{\tau}(E)$ as

$$
\mathcal{I}_{\tau}(E)=\int_{\partial E} \tau\left(n_{E}(x)\right) \mathcal{H}^{d-1}(d x)
$$

where $\mathcal{H}^{d-1}$ denotes the Hausdorff measure in dimension $d-1$. The quantity $\mathcal{I}_{\tau}(E)$ represents the surface tension of $E$ for the norm $\tau$. At the point $x$, the tension has intensity $\tau\left(n_{E}(x)\right)$ in the direction of the normal unit exterior vector $n_{E}(x)$. We denote by $\mathcal{L}^{d}$ the $d$-dimensional Lebesgue measure. We can associate with the norm $\tau$ the following isoperimetric problem:

$$
\operatorname{minimize} \frac{\mathcal{I}_{\tau}(E)}{\mathcal{L}^{d}(E)} \text { subject to } \mathcal{L}^{d}(E) \leq 1 .
$$

We use the Wulff construction to build a minimizer for this anisotropic isoperimetric problem. We define the set $\widehat{W}_{\tau}$ as

$$
\widehat{W}_{\tau}=\bigcap_{v \in \mathbb{S}^{d-1}}\left\{x \in \mathbb{R}^{d}: x \cdot v \leq \tau(v)\right\},
$$

where denotes the standard scalar product and $\mathbb{S}^{d-1}$ is the unit sphere of $\mathbb{R}^{d}$. The set $\widehat{W}_{\tau} / \mathcal{L}^{d}\left(\widehat{W}_{\tau}\right)$ is a minimizer for the isoperimetric problem associated with $\tau$. We will build in section 3 an appropriate norm $\beta_{p}$ for our problem that will be directly related to the open edge boundary ratio. We will denote by $\mathcal{I}_{p}$ the surface tension 
associated with $\beta_{p}$. We define the Wulff crystal $W_{p}$ as the dilate of $\widehat{W}_{\beta_{p}}$ such that $\mathcal{L}^{d}\left(W_{p}\right)=1 / \theta_{p}$, where $\theta_{p}=\mathbb{P}\left(0 \in \mathcal{C}_{\infty}\right)$.

In this paper, we adapt the proof of Gold to any dimension $d \geq 2$ to give a self-contained proof of the existence of the limit for the anchored isoperimetric profile. Note that this proof also holds in dimension 2, it gives an alternative proof of Biskup et al. (2015) with a simpler norm. The interest of this result is to prove the existence of the isoperimetric constant for a more natural definition. Although proving the existence of the anchored isoperimetric profile involves more technical difficulties, the anchored isoperimetric profile itself is a simpler object to study. Cerf and Dembin studied the anchored isoperimetric profile $\varphi_{n}$ at $p_{c}$ in Cerf and Dembin (2020). Studying the behavior of $\varphi_{\mathcal{C}_{n}}$ at $p_{c}$ would have required much more work. The aim of this paper is the proof of the two following Theorems. The first theorem asserts the existence of the limit of $n \varphi_{n}$.

Theorem 1.1. Let $d \geq 2, p>p_{c}(d)$ and let $\beta_{p}$ be the norm that will be properly defined in section 3. Let $W_{p}$ be the Wulff crystal for this norm, i.e., the dilate of $\widehat{W}_{\beta_{p}}$ such that $\mathcal{L}^{d}\left(W_{p}\right)=1 / \theta_{p}$. Then, conditionally on $\left\{0 \in \mathcal{C}_{\infty}\right\}$,

$$
\lim _{n \rightarrow \infty} n \varphi_{n}=\frac{\mathcal{I}_{p}\left(W_{p}\right)}{\theta_{p} \mathcal{L}^{d}\left(W_{p}\right)}=\mathcal{I}_{p}\left(W_{p}\right) \text { a.s.. }
$$

The second theorem shows that the graphs $G_{n}$ realizing the minimum converge in probability towards a translate of $W_{p}$.

Theorem 1.2. Let $d \geq 2$ and $p>p_{c}(d)$. Let $\varepsilon>0$. There exists positive constants $C_{1}$ and $C_{2}$ depending on $d, p$ and $\varepsilon$ such that, for all $n \geq 1$,

$$
\mathbb{P}\left(\max _{G_{n} \in \mathcal{G}_{n}} \inf _{x \in \mathbb{R}^{d}} \frac{1}{n^{d}}\left|G_{n} \Delta\left(n\left(x+W_{p}\right) \cap \mathcal{C}_{\infty}\right)\right| \geq \varepsilon \mid 0 \in \mathcal{C}_{\infty}\right) \leq C_{1} \mathrm{e}^{-C_{2} n^{1-3 / 2 d}},
$$

where $\Delta$ denotes the symmetric difference.

Remark 1.3. We emphasize the fact that when we restrict theorems 1.1 and 1.2 to dimension two, we obtain a weaker version of already existing results. Namely, in Biskup et al. (2015), the authors used methods specific to dimension two in order to derive a uniform convergence in the shape theorem. Whereas here, we only obtain $L^{1}$-convergence.

To prove Theorem 1.1, we first prove a large deviations result from above for $n \varphi_{n}$ stated in the following Theorem.

Theorem 1.4. Let $d \geq 2$. Let $p>p_{c}(d)$. For all $\varepsilon>0$, there exist positive constants $C_{1}$ and $C_{2}$ depending on $p, d$, $\varepsilon$ such that, for all $n \geq 1$,

$$
\mathbb{P}\left(n \varphi_{n} \geq(1+\varepsilon) \frac{\mathcal{I}_{p}\left(W_{p}\right)}{\theta_{p}(d) \mathcal{L}^{d}\left(W_{p}\right)} \mid 0 \in \mathcal{C}_{\infty}\right) \leq C_{1} \exp \left(-C_{2} n\right) .
$$

The proof of Theorem 1.4 is inspired by the proof of Theorem 5.4 in Gold (2018). We shall build a valid subgraph that has an isoperimetric ratio close to $\varphi_{n}$. In order to do so, we approximate the Wulff shape $W_{p}$ from the inside by a convex polytope $P$. We shall build a cutset $\Gamma_{n}$ that cuts $n P$ from infinity whose number of open edges is close to $n^{d-1} \mathcal{I}_{p}(P)$ with high probability. For each face $F$ of $P$ and $v$ its associated exterior unit normal vector, we consider the $\operatorname{cylinder} \operatorname{cyl}(F+\varepsilon v, \varepsilon)$ of basis $F+\varepsilon v$ and of height $\varepsilon>0$. We build $E$ by merging the cutsets from the top to the bottom of minimal capacity of the cylinders $\operatorname{cyl}(F+\varepsilon v, \varepsilon)$. The union of 
these cutsets is not yet a cutset itself because of the potential holes between these cutsets. We fix this issue by adding extra edges to fill the holes. We control next the number of extra edges we have added. We also need to control the capacity of the cutsets in a cylinder of polyhedral basis. We next build a valid subgraph $H_{n} \subset \mathbb{Z}^{d}$ from $\Gamma_{n}$ by taking all the vertices of $\mathcal{C}_{\infty} \cap n P$ that are connected to 0 without using edges in $\Gamma_{n}$. We expect that $\left|H_{n}\right|$ is of order $\theta_{p}(d) n^{d} \mathcal{L}^{d}(P)$. We can bound $\left|\partial_{\mathcal{C}_{\infty}} H_{n}\right|$ from above thanks to the number of open edges in $\Gamma_{n}$ and so we control the isoperimetric ratio of $H_{n}$. Finally, we control the upper large deviations for this number of open edges thanks to the upper large deviations for the flow in a cylinder of polyhedral basis. The next step is to obtain the large deviations result from below.

Theorem 1.5. Let $d \geq 2$. Let $p>p_{c}(d)$. For all $\varepsilon>0$, there exist positive constants $C_{1}$ and $C_{2}$ depending on $p, d$, $\varepsilon$ such that, for all $n \geq 1$,

$$
\mathbb{P}\left(n \varphi_{n} \leq(1-\varepsilon) \frac{\mathcal{I}_{p}\left(W_{p}\right)}{\theta_{p}(d) \mathcal{L}^{d}\left(W_{p}\right)} \mid 0 \in \mathcal{C}_{\infty}\right) \leq C_{1} \exp \left(-C_{2} n^{1-3 / 2 d}\right) .
$$

Remark 1.6. The deviation order in Theorem 1.5 is not optimal due to technical details of the proof. In this work we do not make any attempt to get the proper order of deviation. Our aim is mainly to obtain Theorems 1.1 and 1.2. The study of the large deviations order would be an interesting problem in itself.

Theorem 1.1 follows from Theorem 1.4 and Theorem 1.5 by a straightforward application of the Borel-Cantelli Lemma. Proving the large deviations result from below is the most difficult part of this work. To be able to compare discrete objects with continuous ones, we shall encode each optimizer $G_{n} \in \mathcal{G}_{n}$ as a measure $\mu_{n}$ defined as

$$
\mu_{n}=\frac{1}{n^{d}} \sum_{x \in V\left(G_{n}\right)} \delta_{x / n} .
$$

We first need to build from a minimizer $G_{n}$ an appropriate continuous object $P_{n}$. To do so, we use the same method as in Gold (2018). The main issue is that the boundary of $G_{n}$ may be very tangled, we will have to build a smoother boundary of size of order $n^{d-1}$. This will enable us to build a continuous object $P_{n}$ of finite perimeter such that, with high probability, its associated measure is close to $\mu_{n}$ in some sense to be specified later.

Let $F$ be a subset of $\mathbb{R}^{d}$. We define its associated measure $\nu_{F}$ :

$$
\forall E \in \mathcal{B}\left(\mathbb{R}^{d}\right), \quad \nu_{F}(E)=\theta_{p} \mathcal{L}^{d}(F \cap E) .
$$

We now define the set $\mathcal{W}$ of the measures associated with the translates of the Wulff shape as

$$
\mathcal{W}=\left\{\nu_{x+W_{p}}: x \in \mathbb{R}^{d}\right\} .
$$

Note that $\mu_{n}$ belongs to $\mathcal{M}\left(\mathbb{R}^{d}\right)$, the set of finite measures on $\mathbb{R}^{d}$. We cannot use a metric as in Gold (2018) where $\mu_{n}$ was a measure on $[-1,1]^{d}$. In fact, we will not use a metric here. We first show that all the minimizers $G_{n} \in \mathcal{G}_{n}$ are with high probability in a local neighborhood of $\mathcal{W}$ for a weak topology. This is the key step before proving Theorem 1.5.

Theorem 1.7. Let $d \geq 2$ and $p>p_{c}(d)$. Let $\left.u:\right] 0,+\infty[\rightarrow] 0,+\infty[$ be a nondecreasing function such that $\lim _{t \rightarrow 0} u(t)=0$. For all $\zeta>0$, there exist positive 
constants $C_{1}$ and $C_{2}$ depending on $d, p, u$ and $\zeta$ such that for all $n \geq 1$, for any finite set $\mathfrak{F}_{n}$ of uniformly continuous functions that satisfies:

$$
\forall f \in \mathfrak{F}_{n} \quad\|f\|_{\infty} \leq 1 \quad \text { and } \quad \forall x, y \in \mathbb{R}^{d} \quad|f(x)-f(y)| \leq u\left(\|x-y\|_{2}\right),
$$

we have

$$
\mathbb{P}\left(\exists G_{n} \in \mathcal{G}_{n}, \forall \nu \in \mathcal{W}, \sup _{f \in \widetilde{F}_{n}}\left|\mu_{n}(f)-\nu(f)\right|>\zeta \mid 0 \in \mathcal{C}_{\infty}\right) \leq C_{1} \mathrm{e}^{-C_{2} n^{1-3 / 2 d}} .
$$

The main difficulty of this paper lies in the proof of this theorem. In our context, an issue that was not present in Gold (2018) arises. Whereas the support of the measure $\mu_{n}$ was included in a fixed compact set in Gold (2018), this is not the case here because we do not constrain $G_{n} \in \mathcal{G}_{n}$ to remain in the box $[-n, n]^{d}$. To fix this issue, we will use the method developed in Cerf (2006). We will first localize the set $G_{n}$ in a finite number of balls of radius of order $n$ up to a set of small fractional volume. We will study $G_{n}$ only inside these balls, i.e., the intersection of $G_{n}$ with these balls. The intersection of $G_{n}$ with the boundary of these balls will create an additional surface tension. However, this surface tension is not related to the open boundary edges of $G_{n}$ but to the fact that we have cut $G_{n}$ along these boundaries. Therefore, we should not take this surface tension into account for the isoperimetric constant. In fact, we will cut $G_{n}$ in such a way to ensure that we do not create too much surface tension, i.e., we will cut in regions where $G_{n}$ is not concentrated. To conclude, we will link the probability that the measure $\mu_{n}$ corresponding to $G_{n} \in \mathcal{G}_{n}$ is far from a weak neighborhood of $\mathcal{W}$ with the probability that the surface tension of $G_{n}$ is locally abnormally small.

Finally, to prove Theorem 1.2, we exhibit a set $\mathcal{F}_{n}$ of uniformly continuous functions such that we can bound from above the symmetric difference $\mid G_{n} \Delta(n(x+$ $\left.\left.W_{p}\right) \cap \mathcal{C}_{\infty}\right) \mid$ by $\sup _{f \in \mathfrak{F}_{n}}\left|\mu_{n}(f)-\nu(f)\right|$ for some $\nu \in \mathcal{W}$ and then apply the result of Theorem 1.7.

The rest of the paper is organized as follows. In section 2, we give some definitions and useful results. We do the construction of the norm $\beta_{p}$ in section 3. In section 4 , we prove the upper large deviations in Theorem 1.4. We build a continuous object $P_{n}$ from a minimizer $G_{n} \in \mathcal{G}_{n}$ and prove that its associated measure is close in some sense to the measure $\mu_{n}$ of $G_{n}$ in section 5. Finally, in section 6, we prove Theorem 1.7 that is a preliminary work before proving the lower large deviations Theorem 1.5 and the convergence of $G_{n}$ properly rescaled towards a limit shape in Theorem 1.2.

\section{Some definitions and useful results}

2.1. Geometric notations. For $x=\left(x_{1}, \ldots, x_{d}\right)$, we define

$$
\|x\|_{2}=\sqrt{\sum_{i=1}^{d} x_{i}^{2}} \quad \text { and } \quad\|x\|_{\infty}=\max _{1 \leq i \leq d}\left|x_{i}\right| .
$$

We say that $x, y \in \mathbb{Z}^{d}$ are $*$-connected if $\|x-y\|_{\infty}=1$. We say that $\gamma=\left(x_{0}, \ldots, x_{n}\right)$ is an $*$-path of $\mathbb{Z}^{d}$ if for any $0 \leq i \leq n-1$, the points $x_{i}$ and $x_{i+1}$ belong to $\mathbb{Z}^{d}$ and are $*$-connected. We say that $\Gamma$ is $*$-connected or a lattice animal if any $x, y \in \Gamma$ are connected by an $*$-path in $\Gamma$. We denote by Animals $s_{x}$ the set of lattice animals containing the point $x \in \mathbb{Z}^{d}$. 
Lemma 2.1 (Kesten, 1986, p82 or Grimmett, 1999, p85). Let $x \in \mathbb{Z}^{d}$. For all positive integer $l$,

$$
\mid\left\{\Gamma \in \text { Animals }_{x},|\Gamma|=l\right\} \mid \leq\left(7^{d}\right)^{l} .
$$

Let $S \subset \mathbb{R}^{d}$ and $r>0$, we define $d_{2}(x, S)=\inf _{y \in S}\|x-y\|_{2}$ and $\mathcal{V}(S, r)$ the open $r$-neighborhood of $S$ by

$$
\mathcal{V}(S, r)=\left\{x \in \mathbb{R}^{d}: d_{2}(x, S)<r\right\} .
$$

Let $x \in \mathbb{R}^{d}, r>0$ and a unit vector $v$. We denote by $B(x, r)$ the closed ball of radius $r$ centered at $x$, by $\operatorname{disc}(x, r, v)$ the closed disc centered at $x$ of radius $r$ normal to $v$, and by $B^{+}(x, r, v)$ (respectively $\left.B^{-}(x, r, v)\right)$ the upper (resp. lower) half part of $B(x, r)$ along the direction of $v$, i.e.,

$$
B^{+}(x, r, v)=\{y \in B(x, r):(y-x) \cdot v \geq 0\},
$$

and

$$
B^{-}(x, r, v)=\{y \in B(x, r):(y-x) \cdot v \leq 0\} .
$$

We denote by $\alpha_{d}$ the $\mathcal{L}^{d}$ measure of a unit ball in $\mathbb{R}^{d}$. We denote by $\mathcal{H}^{d-1}$ the Hausdorff measure in dimension $d-1$. In particular, the $\mathcal{H}^{d-1}$ measure of a $d-1$ dimensional unit disc in $\mathbb{R}^{d}$ is equal to $\alpha_{d-1}$. Let $A$ be a non-degenerate hyperrectangle, i.e., a rectangle of dimension $d-1$ in $\mathbb{R}^{d}$. Let $\vec{v}$ be one of the two unit vectors normal to $A$. Let $h>0$, we denote by $\operatorname{cyl}(A, h)$ the cylinder of basis $A$ and height $h$ defined by

$$
\operatorname{cyl}(A, h)=\{x+t \vec{v}: x \in A, t \in[-h, h]\} .
$$

The dependence on $\vec{v}$ is implicit in the notation $\operatorname{cyl}(A, h)$. Note that these definitions of cylinder may be extended in the case where $A$ is of linear dimension $d-1$, i.e., $A$ is included in an hyperplane of $\mathbb{R}^{d}$, which is the affine span of $A$.

2.2. Sets of finite perimeter and surface energy. The perimeter of a Borel set $S$ of $\mathbb{R}^{d}$ in an open set $O$ is defined as

$$
\mathcal{P}(S, O)=\sup \left\{\int_{S} \operatorname{div} f(x) d \mathcal{L}^{d}(x): f \in C_{c}^{\infty}(O, B(0,1))\right\},
$$

where $C_{c}^{\infty}(O, B(0,1))$ is the set of the functions of class $\mathcal{C}^{\infty}$ from $\mathbb{R}^{d}$ to $B(0,1)$ having a compact support included in $O$, and div is the usual divergence operator. The perimeter $\mathcal{P}(S)$ of $S$ is defined as $\mathcal{P}\left(S, \mathbb{R}^{d}\right)$. The topological boundary of $S$ is denoted by $\partial S$. The reduced boundary $\partial^{*} S$ of $S$ is a subset of $\partial S$ such that, at each point $x$ of $\partial^{*} S$, it is possible to define a normal vector $n_{S}(x)$ to $S$ in a measure-theoretic sense, and moreover $\mathcal{P}(S)=\mathcal{H}^{d-1}\left(\partial^{*} S\right)$. Let $\nu$ be a norm on $\mathbb{R}^{d}$. We define its associated Wulff crystal $\mathcal{W}_{\nu}$ as

$$
\mathcal{W}_{\nu}=\left\{x \in \mathbb{R}^{d}: \forall y, y \cdot x \leq \nu(y)\right\} .
$$

With the help of the Wulff crystal, we define the surface energy of a general set.

Definition 2.2. Let $\nu$ be a norm on $\mathbb{R}^{d}$. The surface energy $\mathcal{I}_{\nu}(S, O)$ of a Borel set $S$ of $\mathbb{R}^{d}$ in an open set $O$ is defined as

$$
\mathcal{I}_{\nu}(S, O)=\sup \left\{\int_{S} \operatorname{div} f(x) d \mathcal{L}^{d}(x): f \in C_{c}^{1}\left(O, \mathcal{W}_{\nu}\right)\right\} .
$$


We will note simply $\mathcal{I}_{\nu}(S)=\mathcal{I}_{\nu}\left(S, \mathbb{R}^{d}\right)$.

Proposition 2.3 (Proposition 14.3 in Cerf, 2006). The surface energy $\mathcal{I}_{\nu}(S, O)$ of a Borel set $S$ of $\mathbb{R}^{d}$ of finite perimeter in an open set $O$ is equal to

$$
\mathcal{I}_{\nu}(S, O)=\int_{\partial^{*} S \cap O} \nu\left(n_{S}(x)\right) d \mathcal{H}^{d-1}(x) .
$$

We recall the following fundamental result.

Proposition 2.4 (Isoperimetric inequality). There exist two positive constants $b_{i s o}$, $c_{i s o}$ which depend only on the dimension $d$, such that for any Cacciopoli set $E$, any ball $B(x, r) \subset \mathbb{R}^{d}$,

$$
\begin{gathered}
\min \left(\mathcal{L}^{d}(E \cap B(x, r)), \mathcal{L}^{d}\left(\left(\mathbb{R}^{d} \backslash E\right) \cap B(x, r)\right)\right) \leq b_{i s o} \mathcal{P}(E, \stackrel{\circ}{B}(x, r))^{d / d-1}, \\
\min \left(\mathcal{L}^{d}(E), \mathcal{L}^{d}\left(\mathbb{R}^{d} \backslash E\right)\right) \leq c_{i s o} \mathcal{P}(E)^{d / d-1} .
\end{gathered}
$$

We refer to Giusti (1984), for more details on Cacciopoli sets, isoperimetric inequality and other definitions in geometric measure theory.

2.3. Approximation by convex polytopes. We recall here an important result, which allows us to approximate adequately a set of finite perimeter by a convex polytope.

Definition 2.5 (Convex polytope). Let $P \subset \mathbb{R}^{d}$. We say that $P$ is a convex polytope if there exist $v_{1}, \ldots, v_{m}$ unit vectors and $\varphi_{1}, \ldots, \varphi_{m}$ real numbers such that

$$
P=\bigcap_{1 \leq i \leq m}\left\{x \in \mathbb{R}^{d}: x \cdot v_{i} \leq \varphi_{i}\right\}
$$

We denote by $F_{i}$ the face of $P$ associated with $v_{i}$, i.e.,

$$
F_{i}=P \cap\left\{x \in \mathbb{R}^{d}: x \cdot v_{i}=\varphi_{i}\right\} .
$$

Any convex subset can be approximated from the outside and from the inside by a convex polytope with almost the same surface energy.

Lemma 2.6. Let $\nu$ be a norm on $\mathbb{R}^{d}$. Let $A$ be a bounded convex set. For each $\varepsilon>0$, there exist convex polytopes $P$ and $Q$ such that $P \subset A \subset Q$ and $\mathcal{I}_{\nu}(Q)-\varepsilon \leq$ $\mathcal{I}_{\nu}(A) \leq \mathcal{I}_{\nu}(P)+\varepsilon$.

Proof: Let $A$ be a bounded convex set. Let $\varepsilon>0$. Let $\left(x_{k}\right)_{k \geq 1}$ be a dense family in $\partial A$. For $n \geq 1$, we define $P_{n}$ as the convex hull of $x_{1}, \ldots, x_{n}$, i.e., the smallest convex that contains the points $x_{1}, \ldots, x_{n}$. As $A$ is convex, we have $P_{n} \subset A$ and $P_{n}$ converges towards $A$ when $n$ goes to infinity for the $\mathcal{L}^{1}$ topology. The functional $\mathcal{I}_{\nu}$ is lower semi-continuous, thus

$$
\mathcal{I}_{\nu}(A) \leq \liminf _{n \rightarrow \infty} \mathcal{I}_{\nu}\left(P_{n}\right),
$$

so there exists $n$ large enough such that

$$
\mathcal{I}_{\nu}(A) \leq \mathcal{I}_{\nu}\left(P_{n}\right)+\varepsilon
$$

and we take $P=P_{n}$. The existence of $Q$ was shown in Lemma 5.1. in Cerf and Pisztora (2000) for the Wulff shape. The proof may be easily adapted to a general convex bounded set $A$. 


\section{Construction of the norm}

Minimizing the open edge boundary is the analogue of minimizing a surface tension in the continuous setting. We shall build a norm $\beta_{p}$ that represents the tension that is exerted on the surface, i.e., any point $x$ in a surface $S$ having $n_{S}(x)$ as a normal unit exterior vector has a tension $\beta_{p}\left(n_{S}(x)\right) n_{S}(x)$ that exerts at the point $x$. To build this norm, let us consider $G_{n} \in \mathcal{G}_{n}$. We zoom on the boundary of $G_{n}$, we look at what happens in a small but macroscopic cube centered at a point $x$ in the boundary $\partial G_{n}$ (see Figure 3.1). The cube is located in such a way that its bottom intersects $G_{n}$ and its top intersects $\mathbb{Z}^{d} \backslash G_{n}$, and it is rotated so that its normal vector coincides with the normal exterior vector at the point $x$. As this cube is small, the portion of $G_{n}$ in that cube does not affect much $\left|G_{n}\right|$, the total volume of $G_{n}$. Thus, if one would like to minimize the open edges to volume ratio, one needs to minimize the number of open edges of $\partial G_{n}$ in that cube. This problem is equivalent to finding a set of edges that separates the top from the bottom of the cube with a minimal number of open edges.

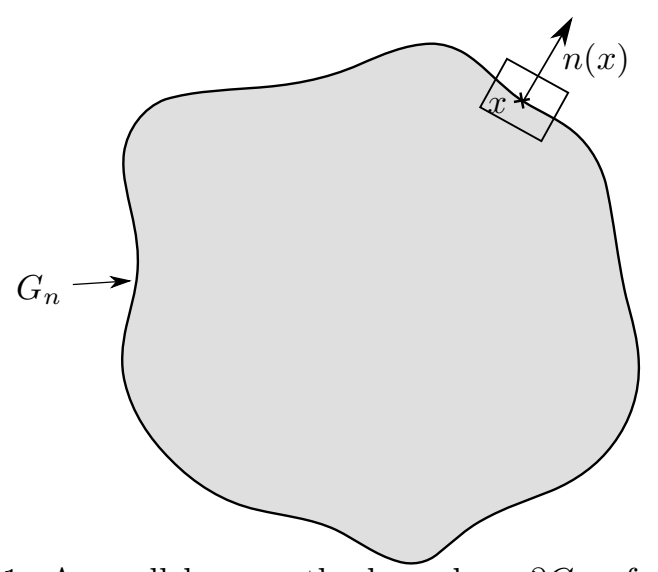

FiguRE 3.1. A small box on the boundary $\partial G_{n}$ of a minimizer $G_{n} \in \mathcal{G}_{n}$

Let us give now a more precise definition of the norm $\beta_{p}$. We consider a bond percolation on $\mathbb{Z}^{d}$ of parameter $p>p_{c}(d)$ with $d \geq 2$. We introduce many notations used for instance in Rossignol and Théret (2010) concerning flows through cylinders. Let $A$ be a non-degenerate hyperrectangle, i.e., a rectangle of dimension $d-1$ in $\mathbb{R}^{d}$. Let $\vec{v}$ be one of the two unit vectors normal to $A$. Let $h>0$, we denote by $\operatorname{cyl}(A, h)$ the cylinder of basis $A$ and height $2 h$ defined by

$$
\operatorname{cyl}(A, h)=\{x+t \vec{v}: x \in A, t \in[-h, h]\} .
$$

The set $\operatorname{cyl}(A, h) \backslash A$ has two connected components, denoted by $C_{1}(A, h)$ and $C_{2}(A, h)$. For $i=1,2$, we denote by $C_{i}^{\prime}(A, h)$ the discrete boundary of $C_{i}(A, h)$ defined by

$$
C_{i}^{\prime}(A, h)=\left\{x \in \mathbb{Z}^{d} \cap C_{i}(A, h): \exists y \notin \operatorname{cyl}(A, h),\langle x, y\rangle \in \mathbb{E}^{d}\right\} .
$$


We say that a set of edges $E$ cuts $C_{1}^{\prime}(A, h)$ from $C_{2}^{\prime}(A, h)$ in $\operatorname{cyl}(A, h)$ if any path $\gamma$ from $C_{1}^{\prime}(A, h)$ to $C_{2}^{\prime}(A, h)$ in $\operatorname{cyl}(A, h)$ contains at least one edge of $E$. We call such a set a cutset. For any set of edges $E$, we denote by $|E|_{o}$ the number of open edges in $E$. We shall call it the capacity of $E$. We define

$$
\tau_{p}(A, h)=\min \left\{|E|_{o}: \quad E \text { cuts } C_{1}^{\prime}(A, h) \text { from } C_{2}^{\prime}(A, h) \text { in } \operatorname{cyl}(A, h)\right\} .
$$

Note that this is a random quantity as $|E|_{o}$ is random, and that the cutsets in this definition are pinned near the boundary of $A$. Finding cutsets of minimal capacity is equivalent to the study of maximal flows, see Bollobás (1979). To each edge $e$, we can associate the random variable $t(e)=\mathbb{1}_{e}$ is open. In the study of maximal flows, we interpret each $t(e)$ as the capacity of the edge $e$, i.e., the maximal amount of water that can flow through $e$ per unit of time. We are interested in the maximal amount of water that can flow through the cylinder given the constraint on the capacity. We refer to Rossignol and Théret (2018) for a rigorous definition of maximal flows. In the following, we will use the term flow to speak about the quantity $\tau_{p}$. The following proposition is a corollary of Proposition 3.5 in Rossignol and Théret (2010), it enables us to give a rigorous definition of the norm $\beta_{p}$.

Proposition 3.1 (Definition of the norm $\left.\beta_{p}\right)$. Let $d \geq 2, p>p_{c}(d), A$ be a nondegenerate hyperrectangle and $\vec{v}$ one of the two unit vectors normal to $A$. Let $h$ an height function such that $\lim _{n \rightarrow \infty} h(n)=\infty$. The limit

$$
\beta_{p}(\vec{v})=\lim _{n \rightarrow \infty} \frac{\mathbb{E}\left[\tau_{p}(n A, h(n))\right]}{\mathcal{H}^{d-1}(n A)}
$$

exists and is finite. Moreover, this limit is independent of $A$ and $h$ and $\beta_{p}$ is a norm.

The norm $\beta_{p}$ is called the flow constant. Roughly speaking, $\beta_{p}(\vec{v})$ corresponds to the expected maximal amount of water that can flow in the direction $\vec{v}$ on average. Actually, we can obtain a stronger convergence. A straightforward application of Theorem 3.8 in Rossignol and Théret (2010) gives the existence of the following almost sure limit:

$$
\lim _{n \rightarrow \infty} \frac{\tau_{p}(n A, h(n))}{\mathcal{H}^{d-1}(n A)}=\beta_{p}(\vec{v}) .
$$

We define

$$
\beta_{\min }=\inf _{\vec{v} \in \mathbb{S}^{d-1}} \beta_{p}(\vec{v}), \quad \beta_{\max }=\sup _{\vec{v} \in \mathbb{S}^{d-1}} \beta_{p}(\vec{v}) .
$$

As $\beta_{p}$ is a norm on $\mathbb{R}^{d}$, we have $\beta_{\min }>0$ and $\beta_{\max }<\infty$. We will need the following upper large deviations result which is a straightforward application of Theorem 4 in Theret (2014).

Theorem 3.2. Let $d \geq 2$ and $p>p_{c}(d)$. For every unit vector $\vec{v}$, for every nondegenerate hyperrectangle $A$ normal to $\vec{v}$, for every $h>0$ and for every $\lambda>\beta_{p}(\vec{v})$, there exist $C_{1}$ and $C_{2}$ depending only on $\lambda$ and $G$, such that, for all $n \geq 0$,

$$
\mathbb{P}\left(\tau_{p}(n A, h n) \geq \lambda \mathcal{H}^{d-1}(A) n^{d-1}\right) \leq C_{1} \exp \left(-C_{2} h n^{d}\right) .
$$

To ease the reading and lighten the notations, the value of the constants may change from appearance to appearance. 


\section{Upper large deviations}

4.1. The case of a cylinder. The aim of this section is to prove Theorem 1.4. A convex polytope of dimension $d-1$ is a convex polytope $F$ which is contained in an hyperplane of $\mathbb{R}^{d}$ and such that $\mathcal{H}^{d-1}(F)>0$. We have the following Lemma.

Lemma 4.1. Let $p>p_{c}(d)$. Let $F$ be a convex polytope of dimension $d-1$. Let $v$ be a unit vector normal to $F$. There exist positive real numbers $C_{1}$ and $C_{2}$ depending on $F, p$ and $d$ such that for all $n \geq 1$, for all $\lambda>\beta_{p}(v) \mathcal{H}^{d-1}(F)$, for all $h>0$

$$
\mathbb{P}\left(\tau_{p}(n F, n h) \geq \lambda n^{d-1}\right) \leq C_{1} \exp \left(-C_{2} h n^{d}\right) .
$$

Proof: Let $p>p_{c}(d)$. Let $F$ be a convex polytope of dimension $d-1$ and $v$ a unit vector normal to $F$. We shall cover $F$ by a finite family of hypersquares and control the probability that the flow is abnormally big in $\operatorname{cyl}(n F, n h)$ by the probability that the flow is abnormally big in one of the cylinders of square basis. Let $\lambda>\beta_{p}(v) \mathcal{H}^{d-1}(F)$. Let $\kappa>0$ be a real number that we will choose later. We denote by $S(\kappa)$ an hypersquare of dimension $d-1$ of side length $\kappa$ and normal to $v$. We want to cover the following region of $F$ by hypersquares isometric to $S(\kappa)$ :

$$
D(\kappa, F)=\{x \in F: d(x, \partial F)>2 \sqrt{d} \kappa\} .
$$

There exists a finite family $\left(S_{i}\right)_{i \in I}$ of closed hypersquares isometric to $S(\kappa)$ included in $F$ having pairwise disjoint interiors, such that $D(\kappa, F) \subset \cup_{i \in I} S_{i}$ (see Figure 4.2). Moreover, there exists a constant $c_{d}$ depending only on the dimension $d$ such that

$$
\mathcal{H}^{d-1}(F \backslash D(\kappa, F)) \leq c_{d} \mathcal{H}^{d-2}(\partial F) \kappa .
$$

We have then

$$
|I| \leq \frac{\mathcal{H}^{d-1}(F)}{\mathcal{H}^{d-1}(S(\kappa))}
$$

Let $h>0$. We would like to build a cutset between $C_{1}^{\prime}(n F, n h)$ and $C_{2}^{\prime}(n F, n h)$ out



Figure 4.2. Covering $P$ with hypersquares 
of minimal cutsets for the flows $\tau_{p}\left(n S_{i}, n h\right), i \in I$. Note that a cutset that achieves the infimum defining $\tau_{p}\left(n S_{i}, n h\right)$ is pinned near the boundary $\partial n S_{i}$. However, if we pick up two hypersquares $S_{i}$ and $S_{j}$ that share a common side, due to the discretization, their corresponding minimal cutsets for the flow $\tau_{p}$ do not necessarily have the same trace on the common face of the associated cylinders $\operatorname{cyl}\left(n S_{i}, n h\right)$ and $\operatorname{cyl}\left(n S_{j}, n h\right)$. We shall fix this problem by adding extra edges around the boundaries of the hypersquares $\partial S_{i}$ in order to glue properly the cutsets. We will need also to add extra edges around $n(F \backslash D(\kappa, F))$ in order to build a cutset between $C_{1}^{\prime}(n F, n h)$ and $C_{2}^{\prime}(n F, n h)$. For $i \in I$, let $E_{i}$ be a minimal cutset for $\tau_{p}\left(n S_{i}, n h\right)$, i.e., $E_{i} \subset \mathbb{E}^{d}$ cuts $C_{1}^{\prime}\left(n S_{i}, n h\right)$ from $C_{2}^{\prime}\left(n S_{i}, n h\right)$ in $\operatorname{cyl}\left(n S_{i}, n h\right)$ and $\left|E_{i}\right|_{o}=\tau_{p}\left(n S_{i}, n h\right)$. We fix $\zeta=4 d$. Let $E_{0}$ be the set of edges of $\mathbb{E}^{d}$ included in $\mathcal{E}_{0}$, where we define

$$
\mathcal{E}_{0}=\left\{x \in \mathbb{R}^{d}: d\left(x, n F \backslash \bigcup_{i \in I} n S_{i}\right) \leq \zeta\right\} \cup \bigcup_{i \in I}\left\{x \in \mathbb{R}^{d}: d\left(x, \partial n S_{i}\right) \leq \zeta\right\} .
$$

The set of edges $E_{0} \cup \bigcup_{i \in I} E_{i}$ separates $C_{1}^{\prime}(n F, n h)$ from $C_{2}^{\prime}(n F, n h)$ in the cylinder $\operatorname{cyl}(n F, n h)$, therefore,

$$
\tau_{p}(n F, n h) \leq\left|E_{0}\right|_{o}+\sum_{i \in I}\left|E_{i}\right|_{o} \leq \operatorname{card}\left(E_{0}\right)+\sum_{i \in I} \tau_{p}\left(n S_{i}, n h\right) .
$$

There exists a constant $c_{d}^{\prime}$ depending only on $d$ such that, using inequalities (4.1) and (4.2),

$$
\begin{aligned}
\operatorname{card}\left(E_{0}\right) & \leq c_{d}^{\prime}\left(\kappa n^{d-1} \mathcal{H}^{d-2}(\partial F)+|I| \mathcal{H}^{d-2}(\partial S(\kappa)) n^{d-2}\right) \\
& \leq c_{d}^{\prime}\left(\kappa n^{d-1} \mathcal{H}^{d-2}(\partial F)+\frac{\mathcal{H}^{d-1}(F)}{\mathcal{H}^{d-1}(S(\kappa))} \mathcal{H}^{d-2}(\partial S(\kappa)) n^{d-2}\right) \\
& \leq c_{d}^{\prime}\left(\kappa n^{d-1} \mathcal{H}^{d-2}(\partial F)+\frac{\mathcal{H}^{d-1}(F)}{\kappa} n^{d-2}\right) .
\end{aligned}
$$

Thus, for $n$ large enough,

$$
\operatorname{card}\left(E_{0}\right) \leq 2 c_{d}^{\prime} \kappa \mathcal{H}^{d-2}(\partial F) n^{d-1} .
$$

There exists $s>0$ such that $\lambda>(1+s) \beta_{p}(v) \mathcal{H}^{d-1}(F)$. We choose $\kappa$ small enough such that

$$
2 c_{d}^{\prime} \kappa \mathcal{H}^{d-2}(\partial F)<\frac{s}{2} \beta_{\text {min }} \mathcal{H}^{d-1}(F) .
$$

Inequalities (4.4) and (4.5) yield that

$$
\operatorname{card}\left(E_{0}\right) \leq \frac{s}{2} \beta_{p}(v) n^{d-1} \mathcal{H}^{d-1}(F) .
$$

Thanks to inequality (4.6), we obtain

$$
\begin{aligned}
\mathbb{P}\left(\tau_{p}(n F, n h) \geq \lambda n^{d-1}\right) & \\
& \leq \mathbb{P}\left(\operatorname{card}\left(E_{0}\right)+\sum_{i \in I} \tau_{p}\left(n S_{i}, n h\right) \geq(1+s) \beta_{p}(v) \mathcal{H}^{d-1}(F) n^{d-1}\right) \\
& \leq \sum_{i \in I} \mathbb{P}\left(\tau_{p}\left(n S_{i}, n h\right) \geq(1+s / 2) \beta_{p}(v) \mathcal{H}^{d-1}\left(S_{i}\right) n^{d-1}\right) .
\end{aligned}
$$


Thanks to Theorem 3.2, there exist positive real numbers $C_{1}, C_{2}$ such that, for all $i \in I$,

$$
\mathbb{P}\left(\tau_{p}\left(n S_{i}, n h\right) \geq(1+s / 2) \beta_{p}(v) \mathcal{H}^{d-1}\left(S_{i}\right) n^{d-1}\right) \leq C_{1} \exp \left(-C_{2} h n^{d}\right) .
$$

By combining inequalities (4.7) and (4.8), we obtain

$$
\mathbb{P}\left(\tau_{p}(n F, n h) \geq \lambda n^{d-1}\right) \leq|I| C_{1} \exp \left(-C_{2} h n^{d}\right),
$$

and the result follows.

We can now proceed to the proof of Theorem 1.4.

Proof of Theorem 1.4: Let $\varepsilon>0$ and $\varepsilon^{\prime}>0$. By Lemma 2.6, there exists a convex polytope $P$ such that $P \subset W_{p}, \mathcal{I}_{p}(P) \leq\left(1+\varepsilon^{\prime}\right) \mathcal{I}_{p}\left(W_{p}\right)$ and $\mathcal{L}^{d}(P) \geq\left(1-\varepsilon^{\prime}\right) \mathcal{L}^{d}\left(W_{p}\right)$. Up to multiplying $P$ by a constant $\alpha<1$ close to 1 , we can assume without loss of generality that $\mathcal{L}^{d}(P)<\mathcal{L}^{d}\left(W_{p}\right)$. We have, for small enough $\varepsilon^{\prime}$ (depending on $\varepsilon$ ),

$$
\begin{aligned}
& \mathbb{P}\left(n \varphi_{n} \geq(1+\varepsilon) \frac{\mathcal{I}_{p}\left(W_{p}\right)}{\theta_{p}(d) \mathcal{L}^{d}\left(W_{p}\right)} \mid 0 \in \mathcal{C}_{\infty}\right) \\
& \quad \leq \mathbb{P}\left(n \varphi_{n} \geq(1+\varepsilon / 2)\left(\frac{1+\varepsilon^{\prime}}{1-\varepsilon^{\prime}}\right) \frac{\mathcal{I}_{p}\left(W_{p}\right)}{\theta_{p}(d) \mathcal{L}^{d}\left(W_{p}\right)} \mid 0 \in \mathcal{C}_{\infty}\right) \\
& \quad \leq \mathbb{P}\left(n \varphi_{n} \geq(1+\varepsilon / 2) \frac{\mathcal{I}_{p}(P)}{\theta_{p}(d) \mathcal{L}^{d}(P)} \mid 0 \in \mathcal{C}_{\infty}\right) .
\end{aligned}
$$

Let us denote by $F_{1}, \ldots, F_{m}$ the faces of $P$ and let $v_{1}, \ldots, v_{m}$ be the associated exterior unit vectors. Let $\delta>0$. For $i \in\{1, \ldots, m\}$, we define

$$
C_{i}=\operatorname{cyl}\left(F_{i}+\delta v_{i}, \delta\right) \text {. }
$$

All the $C_{i}$ are of disjoint interiors because $P$ is convex. Indeed, assume there exists $z \in \stackrel{\circ}{C}_{i} \cap \stackrel{\circ}{C}_{j}$ for some $i \neq j$. Then there exist unique $x \in F_{i}, y \in F_{j}$ and $h, h^{\prime}<2 \delta$ such that $z=x+h v_{i}=y+h^{\prime} v_{j}$. The points $x$ and $y$ correspond to the orthogonal projection of $z$ on $P$. As $P$ is convex, the orthogonal projection on $P$ is unique and so $x=y=z$. This contradicts the fact that $z$ belongs to the interior of $C_{i}$. We now aim to build a cutset that cuts $n P$ from infinity out of cutsets of minimal capacities for $\tau_{p}\left(n\left(F_{i}+\delta v_{i}\right), n \delta\right), i \in\{1, \ldots, m\}$. The union of these cutsets is not enough to form a cutset from $n P$ to infinity because there are holes between these cutsets. We shall add edges around the boundaries $\partial\left(n\left(F_{i}+\delta v_{i}\right)\right)$ to close these holes (see Figure 4.3). As the distance between two adjacent boundaries $\partial\left(n\left(F_{i}+\delta v_{i}\right)\right)$ decreases with $\delta$, by taking $\delta$ small enough, the size of the bridges and so their capacities are not too big. We recall that the capacity of a set, namely the number of open edges in the set, may be bounded from above by its size. Next, we control the maximal flow through the cylinders or equivalently the capacity of minimal cutsets in the cylinders with the help of Lemma 4.1.

For $i \in\{1, \ldots, m\}$, let $E_{i}^{\prime}$ be a minimal cutset for $\tau_{p}\left(n\left(F_{i}+\delta v_{i}\right), n \delta\right)$, i.e., $E_{i}^{\prime}$ cuts $C_{1}^{\prime}\left(n\left(F_{i}+\delta v_{i}\right), \delta\right)$ from $C_{2}^{\prime}\left(n\left(F_{i}+\delta v_{i}\right), \delta\right)$ and $\left|E_{i}^{\prime}\right|_{o}=\tau_{p}\left(n\left(F_{i}+\delta v_{i}\right), \delta n\right)$. We shall add edges to control the space between $E_{i}^{\prime}$ and the boundary $\partial\left(n\left(F_{i}+\delta v_{i}\right)\right)$. Let $\zeta=4 d$. Let $i, j \in\{1, \ldots, m\}$ such that $F_{i}$ and $F_{j}$ share a common side. We define

$$
\mathcal{M}(i, j)=\left(\mathcal{V}\left(n F_{i} \cap n F_{j}, n \delta+\zeta\right) \backslash \mathcal{V}\left(n F_{i} \cap n F_{j}, n \delta-\zeta\right)\right) \cap(n P)^{c} .
$$


Let $M_{i, j}$ denote the set of the edges in $\mathbb{E}_{n}^{d}$ included in $\mathcal{M}(i, j)$ (see Figure 4.3). There exists a constant $c_{d}^{\prime}$ depending only on the dimension $d$ such that for all $i, j \in\{1, \ldots, m\}$ such that $F_{i}$ and $F_{j}$ share a common side,

$$
\operatorname{card}\left(M_{i, j}\right) \leq c_{d} \delta^{d-1} n^{d-1} .
$$

We set

$$
M=\bigcup_{i, j} M_{i, j},
$$

where the union is over $i, j \in\{1, \ldots, m\}$ such that $i \neq j$ and $F_{i}, F_{j}$ share a common side. The set $\Gamma_{n}=M \cup \bigcup_{i=1}^{m} E_{i}^{\prime}$ cuts $n P$ from infinity. We define $H_{n}$ to be the set of the vertices connected to 0 by open paths which do not use an edge of $\Gamma_{n}$, i.e.,

$$
H_{n}=\left\{x \in \mathbb{Z}^{d}, x \text { is connected to } 0 \text { with open edges in } \mathbb{E}^{d} \backslash \Gamma_{n}\right\} \text {. }
$$



Figure 4.3. Construction of a cutset $\Gamma_{n}$ from $n P$ to infinity

By definition, the set $H_{n}$ is connected. As we condition on the event $\left\{0 \in \mathcal{C}_{\infty}\right\}$, the set $H_{n}$ is a subgraph of $\mathcal{C}_{\infty}$. As $P$ is a polytope,

$$
\mathcal{I}_{p}(P)=\sum_{i=1}^{m} \beta_{p}\left(v_{i}\right) \mathcal{H}^{d-1}\left(F_{i}\right) .
$$

Moreover, we have

$$
\left|\partial_{\mathcal{C}_{\infty}} H_{n}\right|=\left|\partial^{o} H_{n}\right| \leq\left|\Gamma_{n}\right|_{o}
$$

where the last inequality comes from the fact that, by construction of $H_{n}$, if $e \in$ $\partial H_{n} \backslash \Gamma_{n}$, then $e$ is necessarily closed. Using (4.10), we have

$$
\begin{aligned}
\left|\Gamma_{n}\right|_{o} & \leq \operatorname{card}(M)+\sum_{i=1}^{m}\left|E_{i}^{\prime}\right|_{o} \\
& \leq c_{d} m^{2} \delta^{d-1} n^{d-1}+\sum_{i=1}^{m} \tau_{p}\left(n\left(F_{i}+\delta v_{i}\right), \delta n\right) .
\end{aligned}
$$


We choose $\delta$ small enough so that

$$
m^{2} c_{d} \delta^{d-1}<\delta \mathcal{I}_{p}(P) / 2 \quad \text { and } \quad \mathcal{L}^{d}(\mathcal{V}(\partial P, 3 \delta)) \leq \delta \mathcal{L}^{d}(P) .
$$

Let us now estimate the probability that $\left|\Gamma_{n}\right|_{o}$ is abnormally big. Using inequalities (4.11) and (4.12), we get

$$
\begin{aligned}
& \mathbb{P}\left(\left|\Gamma_{n}\right|_{o} \geq(1+\delta) \mathcal{I}_{p}(P) n^{d-1} \mid 0 \in \mathcal{C}_{\infty}\right) \\
& \leq \frac{1}{\theta_{p}} \mathbb{P}\left(\operatorname{card}(M)+\sum_{i=1}^{m} \tau_{p}\left(n\left(F_{i}+\delta v_{i}\right), \delta n\right) \geq(1+\delta) \sum_{i=1}^{m} \beta_{p}\left(v_{i}\right) \mathcal{H}^{d-1}\left(F_{i}\right) n^{d-1}\right) \\
& \leq \frac{1}{\theta_{p}} \mathbb{P}\left(\sum_{i=1}^{m} \tau_{p}\left(n\left(F_{i}+\delta v_{i}\right), \delta n\right) \geq(1+\delta / 2) \sum_{i=1}^{m} \beta_{p}\left(v_{i}\right) \mathcal{H}^{d-1}\left(F_{i}\right) n^{d-1}\right) \\
& \quad \leq \frac{1}{\theta_{p}} \sum_{i=1}^{m} \mathbb{P}\left(\tau_{p}\left(n\left(F_{i}+\delta v_{i}\right), \delta n\right) \geq(1+\delta / 2) \beta_{p}\left(v_{i}\right) \mathcal{H}^{d-1}\left(F_{i}\right) n^{d-1}\right) .
\end{aligned}
$$

By Lemma 4.1, there exist positive constants $C_{1}, C_{2}$ depending on $d, p, P$ and $\delta$ such that, for all $1 \leq i \leq m$,

$$
\mathbb{P}\left(\tau_{p}\left(n\left(F_{i}+\delta v_{i}\right), \delta n\right) \geq(1+\delta / 2) \beta_{p}\left(v_{i}\right) \mathcal{H}^{d-1}\left(F_{i}\right) n^{d-1}\right] \leq C_{1} \exp \left(-C_{2} \delta n^{d}\right) .
$$

Finally, combining inequalities (4.13) and (4.14), we obtain

$$
\mathbb{P}\left(\left|\Gamma_{n}\right|_{o} \geq(1+\delta) \mathcal{I}_{p}(P) n^{d-1}\right) \leq \frac{m C_{1}}{\theta_{p}} \exp \left(-C_{2} \delta n^{d}\right) .
$$

We shall now estimate the number of vertices in $H_{n}$ in order to check that $H_{n}$ is a valid subgraph. For that purpose, we use a renormalization argument. Let $k>0$. We partition $\mathbb{R}^{d}$ into disjoint cubes of side length $1 / k$. We define $B_{j}^{\prime}$ as the union of $B_{j}$ and all its $3^{d}-1 *$-neighbors (the cubes $B$ having at least one vertex at $L^{1}$ distance less than 1 from $B_{j}$ ). We consider $B_{1}, \ldots, B_{l_{1}}$ the cubes such that $B_{1}^{\prime}, \ldots, B_{l_{1}}^{\prime}$ are contained in $P \backslash \mathcal{V}(\partial P, 2 \delta)$ and $B_{l_{1}+1}, \ldots, B_{l_{2}}$ the cubes such that $B_{l_{1}+1}^{\prime}, \ldots, B_{l_{2}}^{\prime}$ intersect $\mathcal{V}(\partial P, 2 \delta)$. We can choose $k$ large enough such that

$$
\mathcal{L}^{d}\left(\bigcup_{i=l_{1}+1}^{l_{2}} B_{i}\right) \leq \mathcal{L}^{d}(\mathcal{V}(\partial P, 3 \delta)) \leq \delta \mathcal{L}^{d}(P) .
$$

We say that a cube $B_{j}$ is good if the following event $\mathcal{E}_{n}^{(j)}$ occurs:

- There exists a unique open cluster of diameter larger than $n / k$ in $n B_{j}^{\prime}$.

- We have $\frac{\left|\mathcal{C}_{\infty} \cap n B_{j}\right|}{\mathcal{L}^{d}\left(n B_{j}\right)} \in\left(\theta_{p}-\delta, \theta_{p}+\delta\right)$.

There exist positive constants $C_{1}$ and $C_{2}$ depending on $d, p, k$ and $\delta$ such that

$$
\mathbb{P}\left(\mathcal{E}_{n}^{(j) c}\right) \leq C_{1} \exp \left(-C_{2} n\right) .
$$

For a proof of the control of the probability of the first property see Theorem 7.68 in Grimmett (1999) or Pisztora (1996), for the second property see Pisztora (1996). If the cube $B_{j}$ is good, we denote by $C_{j}$ its unique open cluster of diameter larger than $n / k$ in $n B_{j}^{\prime}$, for $1 \leq j \leq l_{1}$. On the event $\bigcap_{1 \leq j \leq l_{1}} \mathcal{E}_{n}^{(j)} \cap\left\{0 \in \mathcal{C}_{\infty}\right\}$, the set $\bigcup_{j=1}^{l_{1}} C_{j}$ is connected without using edges of $\Gamma_{n}$ and contains 0 , therefore, it is a subgraph of $H_{n}$. Furthermore, we claim that, on this event, we have $\mathcal{C}_{\infty} \cap\left(\bigcup_{1 \leq j \leq l_{1}} n B_{j}\right) \subset H_{n}$. Indeed, let us assume that there exists $x \in \mathcal{C}_{\infty} \cap\left(\bigcup_{1 \leq j \leq l_{1}} n B_{j}\right)$ that does not 
belong to $H_{n}$. Both 0 and $x$ belong to $\mathcal{C}_{\infty}$, therefore, $x$ is connected to 0 by a path $\gamma=\left(x_{0}, e_{1}, \ldots, e_{l}, x_{l}\right)$ with $x_{0}=0$ and $x_{l}=x$ that uses edges in $\Gamma_{n}$. We define

$$
r=\sup \left\{i \geq 1, e_{i} \in \Gamma_{n}\right\} .
$$

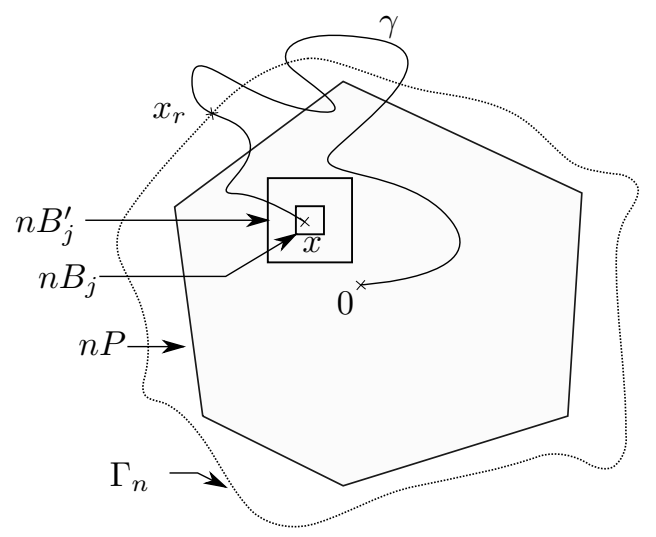

FiguRE 4.4. Vertices in $H_{n}$

By construction, as $e_{l} \notin \Gamma_{n}$, we have $r<l$. Let us denote $\gamma^{\prime}=\left(x_{r}, e_{r+1}, \ldots, x_{l}\right)$. The path $\gamma^{\prime}$ is not connected to $H_{n}$ without using edges in $\Gamma_{n}$ (see Figure 4.4). Let $j$ such that $x \in n B_{j}$, by construction $x_{r}$ is outside $n B_{j}^{\prime}$. Moreover, on the event $\mathcal{E}_{n}^{(j)}$, the cube $n B_{j}^{\prime}$ contains a unique cluster of diameter larger than $n / k$. As the path $\gamma^{\prime}$ starts outside $n B_{j}^{\prime}$ and ends inside $n B_{j}$, its intersection with $n B_{j}^{\prime}$ has a diameter larger than $n / k$. Besides, the path $\gamma^{\prime}$ is not connected to $H_{n}$ in $n B_{j}^{\prime}$ by an open path, so the cube $n B_{j}^{\prime}$ contains two open clusters of diameter larger than $n / k$. This is a contradiction with the first property of a good cube. Therefore, on the event $\bigcap_{1 \leq j \leq l_{1}} \mathcal{E}_{n}^{(j)} \cap\left\{0 \in \mathcal{C}_{\infty}\right\}$,

$$
\begin{aligned}
\left|H_{n}\right| & \geq\left|\mathcal{C}_{\infty} \cap\left(\cup_{1 \leq j \leq l_{1}} n B_{j}\right)\right| \\
& \geq\left(\theta_{p}-\delta\right) \sum_{i=1}^{l_{1}} \mathcal{L}^{d}\left(n B_{i}\right) .
\end{aligned}
$$

Thanks to inequalities (4.16) and (4.18), we obtain

$$
\left|H_{n}\right| \geq\left(\theta_{p}-\delta\right)(1-\delta) \mathcal{L}^{d}(n P) .
$$

To ensure that $H_{n}$ is a valid subgraph, it remains to check that $\left|H_{n}\right| \leq n^{d}$, yet we have

$$
\begin{aligned}
\left|H_{n}\right| & \leq\left(\theta_{p}+\delta\right) \sum_{i=1}^{l_{1}} \mathcal{L}^{d}\left(n B_{i}\right)+\sum_{i=l_{1}+1}^{l_{2}} \mathcal{L}^{d}\left(n B_{i}\right) \\
& \leq\left(\theta_{p}+\delta\right) n^{d} \mathcal{L}^{d}(P)+n^{d} \delta \mathcal{L}^{d}(P) \\
& \leq\left(\theta_{p}+2 \delta\right) n^{d} \mathcal{L}^{d}(P) .
\end{aligned}
$$

As $\mathcal{L}^{d}(P)<\mathcal{L}^{d}\left(W_{p}\right)$, we can choose $\delta$ small enough such that

$$
\left|H_{n}\right| \leq \theta_{p} \mathcal{L}^{d}\left(W_{p}\right) n^{d} \leq n^{d} .
$$


Finally, on the event

$$
\bigcap_{1 \leq j \leq l_{1}} \mathcal{E}_{n}^{(j)} \cap\left\{\left|\Gamma_{n}\right|_{o} \leq(1+\delta) \mathcal{I}_{p}(P) n^{d-1}\right\} \cap\left\{0 \in \mathcal{C}_{\infty}\right\}
$$

combining (4.11) and (4.19), we obtain, for small enough $\delta$,

$$
n \varphi_{n} \leq \frac{\left|\Gamma_{n}\right|_{o}}{\left|H_{n}\right|} \leq(1+\delta) \frac{\mathcal{I}_{p}(P)}{\left(\theta_{p}-2 \delta\right)(1-\delta) \mathcal{L}^{d}(P)} \leq(1+\varepsilon / 2) \frac{\mathcal{I}_{p}(P)}{\theta_{p} \mathcal{L}^{d}(P)} .
$$

Combining the result of Lemma 4.1 and inequalities (4.9), (4.15) and (4.17), we obtain

$$
\begin{aligned}
\mathbb{P}\left(n \varphi_{n} \geq(1+\varepsilon) \frac{\mathcal{I}_{p}\left(W_{p}\right)}{\theta_{p}(d) \mathcal{L}^{d}\left(W_{p}\right)} \mid 0\right. & \left.\in \mathcal{C}_{\infty}\right) \\
& \leq \frac{l_{1} C_{1}}{\theta_{p}} \exp \left(-C_{2} n\right)+\frac{m C_{1}}{\theta_{p}} \exp \left(-C_{2} \delta n^{d}\right)
\end{aligned}
$$

This yields the result.

\section{Construction of a continuous object}

The aim of this section is to build a continuous object $P_{n}$ from a minimizer $G_{n} \in \mathcal{G}_{n}$

5.1. Some useful results on the minimizers. The following lemma ensures that the size of the minimizers $G_{n} \in \mathcal{G}_{n}$ are of order $n^{d}$.

Lemma 5.1. Let $d \geq 2$ and $p>p_{c}(d)$. There exist positive constants $D_{1}, D_{2}$ and $\eta_{1}$ depending only on $d$ and $p$ such that, for all $n \geq 1$,

$$
\mathbb{P}\left(\exists G_{n} \in \mathcal{G}_{n},\left|G_{n}\right| \leq \eta_{1} n^{d} \mid 0 \in \mathcal{C}_{\infty}\right) \leq D_{1} \exp \left(-D_{2} n^{(d-1) / 2 d)}\right) .
$$

To prove Lemma 5.1, we adapt the proof of Lemma A.8 in Gold (2018). We need the following proposition that ensures that the open edge boundary of a large subgraph is not too small.

Proposition 5.2 (Berger-Biskup-Hoffman-Kozma, Proposition 5.2. in Berger et al., 2008). Let $d \geq 2$ and $p>p_{c}(d)$. There exist positive constants $c_{1}, c_{2}$ and $c_{3}$ depending only on $d$ and $p$ such that, for all $t \geq 0$,

$$
\mathbb{P}\left(\begin{array}{c}
\text { There exists an open connected graph containing } 0 \\
\text { such that }|G| \geq t^{d /(d-1)},\left|\partial^{o} G\right| \leq c_{3}|G|^{(d-1) / d}
\end{array}\right) \leq c_{1} \exp \left(-c_{2} t\right) .
$$

Proof of Lemma 5.1: Thanks to Theorem 1.4, there exist positive constants $c_{1}^{\prime}, c_{2}^{\prime}$ and $c_{3}^{\prime}$ depending only on $p$ and $d$ such that for all $n \geq 1$,

$$
\mathbb{P}\left(\varphi_{n} \geq c_{3}^{\prime} n^{-1} \mid 0 \in \mathcal{C}_{\infty}\right) \leq c_{1}^{\prime} \exp \left(-c_{2}^{\prime} n\right)
$$

Let $G_{n} \in \mathcal{G}_{n}$. If $\left|G_{n}\right| \leq \sqrt{n}$, as $G_{n} \subset \mathcal{C}_{\infty}$ the set $\partial^{\circ} G_{n}$ is non empty on the event $\left\{0 \in \mathcal{C}_{\infty}\right\}$ and so $\varphi_{n} \geq n^{-1 / 2}$. This is impossible for large $n$. We now assume $\left|G_{n}\right|>$ $\sqrt{n}$. Using Proposition 5.2 with $t=n^{(d-1) / 2 d}$, conditioning on $\left\{0 \in \mathcal{C}_{\infty}\right\}$, we obtain that $\left|\partial^{o} G_{n}\right| \geq c_{3}\left|G_{n}\right|^{(d-1) / d}$ with probability at least $1-c_{1} \exp \left(-c_{2} n^{(d-1) / 2 d}\right) / \theta_{p}$. Moreover, on the event $\left\{\varphi_{n} \leq c_{3}^{\prime} n^{-1}\right\} \cap\left\{0 \in \mathcal{C}_{\infty}\right\}$, we obtain

$$
c_{3}\left|G_{n}\right|^{-1 / d} \leq \frac{\left|\partial^{o} G_{n}\right|}{\left|G_{n}\right|}=\varphi_{n} \leq c_{3}^{\prime} n^{-1} \text {. }
$$


So we set $\eta_{1}=\left(c_{3} / c_{3}^{\prime}\right)^{d}$. Finally,

$$
\begin{aligned}
\mathbb{P}\left(\exists G_{n} \in \mathcal{G}_{n},\right. & \left.\left|G_{n}\right| \leq \eta_{1} n^{d} \mid 0 \in \mathcal{C}_{\infty}\right) \\
& \leq \mathbb{P}\left(\varphi_{n} \geq c_{3}^{\prime} n^{-1} \mid 0 \in \mathcal{C}_{\infty}\right)+\frac{c_{1}}{\theta_{p}} \exp \left(-c_{2} n^{(d-1) / 2 d}\right) \\
& \leq c_{1}^{\prime} \exp \left(-c_{2}^{\prime} n\right)+\frac{c_{1}}{\theta_{p}} \exp \left(-c_{2} n^{(d-1) / 2 d}\right)
\end{aligned}
$$

This yields the result.

5.2. Construction of a continuous set. To study the upper large deviations, we needed to go from a continuous object to a discrete object. In this section, we do the opposite. From now on, we will always condition on the event $\left\{0 \in \mathcal{C}_{\infty}\right\}$. We start with $G_{n} \in \mathcal{G}_{n}$ and we build a continuous object $P_{n}$. Our goal is to build a continuous object of finite perimeter which is close to $n^{-(d-1)}\left|\partial^{\circ} G_{n}\right|$. Although it seems natural to take the continuous object $P_{n}=n^{-1}\left(G_{n}+[-1 / 2,1 / 2]^{d}\right)$, this turns out to be a bad choice because the boundary $\partial G_{n}$ may be very tangled and its size may be of higher order than $n^{d-1}$. We will build from $G_{n}$ a graph $F_{n}$ with a smoother boundary $\Gamma_{n} \subset \mathbb{E}^{d}$ in order to build the continuous object $P_{n}$. At this point, there is some work left. If we consider the subgraph $F_{n}$ that contains all the vertices in $\mathcal{C}_{\infty}$ enclosed in $\Gamma_{n}$, the symmetric difference $F_{n} \Delta G_{n}$ may be big due to the presence of holes in $G_{n}$, more precisely portions of $\mathcal{C}_{\infty}$ enclosed in $\Gamma_{n}$ but not contained in $G_{n}$ (see Figure 5.5). Indeed, if these holes are too large, the symmetric difference $F_{n} \Delta G_{n}$ will be large too. However, we cannot keep all the holes in $G_{n}$ to build $F_{n}$ because when we will pass to a continuous object $P_{n}$, these holes will considerably increase the perimeter of $P_{n}$ so that $P_{n}$ may have a too large perimeter. The solution is to fill only the small holes to obtain $F_{n}$ so that the perimeter of $P_{n}$ remains of the correct order and the symmetric difference $F_{n} \Delta G_{n}$ remains small. In order to do so, we shall perform Zhang's construction in Zhang (2018) to obtain a smooth boundary $\Gamma_{n}$ for $G_{n}$ but also to surgically remove these large holes from $G_{n}$ by cutting along a smooth boundary. This work was done in Gold (2018). We will only partially sketch Zhang's construction and we refer to Zhang (2018) for a rigorous proof and more details about the construction. Although we did the same construction as Gold (2018), we do not use the same argument to conclude. Gold used a procedure called webbing to link all the different contours together in order to obtain a single connected object, this simplifies the combinatorial estimates. Here, we do not perform the webbing procedure, instead we use adequate combinatorial estimates. Avoiding the webbing procedure enables us to extend the result to dimension 2 .

Let us define a renormalization process. For a large integer $k$, that will be chosen later, we set $B_{k}=\left[-k, k\left[{ }^{d} \cap \mathbb{Z}^{d}\right.\right.$ and define the following family of $k$-cubes, for $\mathbf{i} \in \mathbb{Z}^{d}$,

$$
B_{k}(\mathbf{i})=\tau_{\mathbf{i}(2 k+1)}\left(B_{k}\right),
$$

where $\tau_{b}$ denotes the shift in $\mathbb{Z}^{d}$ with vector $b \in \mathbb{Z}^{d}$. The lattice $\mathbb{Z}^{d}$ is the disjoint union of this family: $\mathbb{Z}^{d}=\sqcup_{\mathbf{i} \in \mathbb{Z}^{d}} B_{k}(\mathbf{i})$. We introduce larger boxes $B_{k}^{\prime}$, for $\mathbf{i} \in \mathbb{Z}^{d}$, we define

$$
B_{k}^{\prime}(\mathbf{i})=\tau_{\mathbf{i}(2 k+1)}\left(B_{3 k}\right) .
$$

Underscore will be used to denote sets of cubes. For any set of $k$-cubes $\underline{A}$, the set $\underline{A}^{\prime}$ denotes the set of the corresponding $3 k$-cubes. Let $G_{n} \in \mathcal{G}_{n}$. We first use Zhang's 
construction to build a smooth cutset $\Gamma_{n}$ that separates $G_{n}$ from infinity. We denote by $\underline{A}$ the set of $k$-cubes that intersect $\partial_{e} G_{n}$, the exterior edge boundary of $G_{n}$. We then modify the current configuration $\omega$ into a configuration $\omega^{\prime}$ by closing all the open edges in $\partial G_{n}$. This procedure is only formal as we will eventually reopen these edges. Zhang's construction enables us to extract a set of cubes $\underline{\Gamma} \subset \underline{A}$ such that $\underline{\Gamma}$ is $*$-connected and in the configuration $\omega^{\prime}$, the union of the $3 k$-cubes of $\underline{\Gamma}^{\prime}$ contains a closed cutset $\Gamma_{n}$ that isolates $G_{n}$ from infinity and a rare event occurs in every cube of $\underline{\Gamma}$. These rare events are due to the existence of a closed cutset that creates a large interface of closed edges, this is a very unlikely event when $p>p_{c}(d)$. Of course, when we will eventually switch back to the configuration $\omega$, these rare events will not occur anymore in some cubes.

Several connected components of $\mathcal{C}_{\infty} \backslash G_{n}$ in $\mathbb{Z}^{d} \backslash \Gamma_{n}$ are enclosed in $\Gamma_{n}$ (see Figure 5.5). We say that a connected component $C$ of $\mathcal{C}_{\infty}$ is surrounded by $\Gamma_{n}$ if any path from $C$ to infinity has to use an edge of $\Gamma_{n}$. We will say that $C$ is large if $|C| \geq n^{1-1 / 2(d-1)}$. We enumerate the large connected components $L_{1}, \ldots, L_{m}$ and the small connected component $S_{1}, \ldots, S_{N}$. We denote by $m\left(G_{n}\right)$ the number of large connected components of $\mathcal{C}_{\infty} \backslash G_{n}$ enclosed in $\Gamma_{n}$.

Remark 5.3. We insist here on the fact that these large components are not holes of the infinite cluster but holes of $G_{n}$ (see Figure 5.5). Intuitively, we do not expect that a minimizer contains such holes because the graph obtained by filling all these holes have a smaller isoperimetric ratio. Indeed, by filling these holes, we reduce the open edge boundary and increase the volume. However, by filling these holes, the volume may exceed $n^{d}$ and the graph we obtain by filling these holes may not be admissible. That is the reason why we cannot easily discard the presence of these large holes inside $G_{n}$. To obtain the proper order of large deviations, one would have to fix this issue.

We then build $F_{n} \subset \mathcal{C}_{\infty}$ by filling the small connected components $S_{1}, \ldots, S_{N}$ of $G_{n}$, i.e.,

$$
F_{n}=G_{n} \cup \bigcup_{i=1}^{N} S_{i} .
$$

At this point, the boundary $\partial F_{n} \backslash \partial_{e} F_{n}$ of $F_{n}$ may be still tangled around the large components. In the configuration $\omega^{\prime}$, for each $1 \leq j \leq m$, there exists a closed cutset that separates $L_{j}$ from infinity. We can apply Zhang's construction to each component $L_{j}$ in order to build a smooth closed cutset $\widehat{\Gamma}_{n}^{(j)}$ and its corresponding set of $k$-cubes $\underline{\Gamma}_{n}^{(j)}$. Thanks to Zhang's construction, the set of cubes $\underline{\Gamma}_{n}^{(j)}$ is *connected and in the configuration $\omega^{\prime}$, a rare event occurs in each of its cubes. We denote the boundary of $F_{n}$ by $\widetilde{\Gamma}_{n}$ and its associated set of $k$-cubes $\underline{\Gamma}_{n}$ as

$$
\widetilde{\Gamma}_{n}=\Gamma_{n} \cup \bigcup_{i=1}^{m} \widehat{\Gamma}_{n}^{(i)}, \quad \underline{\widetilde{\Gamma}}_{n}=\underline{\Gamma}_{n} \cup \bigcup_{i=1}^{m} \underline{\widehat{\Gamma}}_{n}^{(i)} .
$$

The set of $k$-cubes $\widetilde{\Gamma}_{n}$ is not $*$-connected. It only contains cubes where a rare event occurs in the configuration $\omega^{\prime}$. Although for some cubes these events do not occur anymore in the configuration $\omega$, we can bound from below the number of cubes that remain unchanged by $\left|\widetilde{\Gamma}_{n}\right|-\left|\partial^{\circ} G_{n}\right|$. In these cubes, rare events still occur when we switch back to the original configuration $\omega$. Using a Peierls argument, we can deduce that, with high probability, $\left|\underline{\Gamma}_{n}\right|$ and $\left|\partial^{\circ} G_{n}\right|$ are of same order when $k$ is 


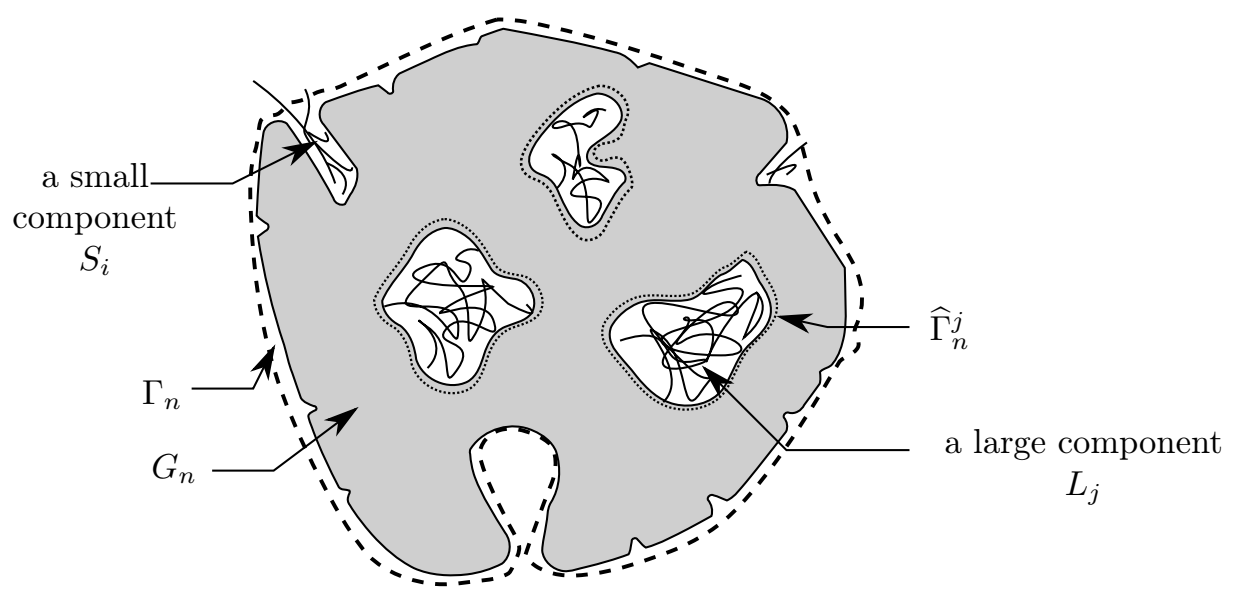

FiguRE 5.5. Construction of $\widetilde{\Gamma}_{n}$ for a $G_{n} \in \mathcal{G}_{n}$

taken large enough. To perform the combinatorial estimates we will need the two following propositions.

Proposition 5.4 (Lemmas 6 and 7 in Zhang, 2018). Let $d \geq 2$ and let $p>p_{c}(d)$. There exist positive constants $C_{1}$ and $C_{2}$ depending only on $p$ and $d$ such that for each $k$-cube $B_{k}$,

$$
\mathbb{P}\left(\text { a rare event occurs in } B_{k}\right) \leq C_{1} \mathrm{e}^{-C_{2} k} .
$$

Moreover, this rare event depends only on the configuration of the $3 k$-cube $B_{k}^{\prime}$.

Remark 5.5. We do not define here what these rare events are, we refer to Zhang (2018) for a precise definition of these rare events. For our purpose we only need to know that the decay is exponential in $k$. We say that a cube is abnormal if a rare event occurs in this cube.

Proposition 5.6. Let $d \geq 2$ and $p>p_{c}(d)$. There exist positive constants $c_{1}, c_{2}$ and $c_{3}$ such that

$$
\mathbb{P}\left(\exists G_{n} \in \mathcal{G}_{n}, m\left(G_{n}\right)>c_{3} n^{d-2+3 / 2 d} \mid 0 \in \mathcal{C}_{\infty}\right) \leq c_{1} \exp \left(-c_{2} n^{1-3 / 2 d}\right) .
$$

Proof: Thanks to Theorem 1.4, there exist positive constants $C_{1}^{\prime}, C_{2}^{\prime}$ and $C_{3}^{\prime}$ depending only on $p$ and $d$ such that for all $n \geq 1$,

$$
\mathbb{P}\left(\varphi_{n} \geq C_{3}^{\prime} n^{-1} \mid 0 \in \mathcal{C}_{\infty}\right) \leq C_{1}^{\prime} \exp \left(-C_{2}^{\prime} n\right) .
$$

Let $G_{n} \in \mathcal{G}_{n}$. We have with probability at least $1-C_{1}^{\prime} \exp \left(-C_{2}^{\prime} n\right)$ that

$$
\left|\partial^{o} G_{n}\right| \leq C_{3}^{\prime} n^{-1}\left|G_{n}\right| \leq C_{3}^{\prime} n^{d-1} .
$$

Thanks to Proposition 5.2, there exist positive constants $c_{1}^{\prime}, c_{2}^{\prime}$ and $c_{3}^{\prime}$ depending only on $p$ and $d$ such that, for all $t \geq 0$, we have

$$
\mathbb{P}\left(\begin{array}{c}
\text { There exists an open connected graph containing } 0 \\
\text { such that }|G| \geq t^{d /(d-1)},\left|\partial^{\circ} G\right| \leq c_{3}^{\prime}|G|^{(d-1) / d}
\end{array}\right) \leq c_{1}^{\prime} \exp \left(-c_{2}^{\prime} t\right) .
$$


In the following, we set $t=n^{(1-1 / 2(d-1))(d-1) / d}=n^{1-3 / 2 d}$. First notice that by construction, each $L_{j}$ is contained in $\left[-n^{d}, n^{d}\right] \cap \mathbb{Z}^{d}$. We have

$$
\begin{aligned}
& \mathbb{P}\left(\exists G_{n} \in \mathcal{G}_{n}, \exists i \in\left\{1, \ldots, m\left(G_{n}\right)\right\},\left|\partial^{o} L_{i}\right| \leq c_{3}^{\prime} n^{(1-1 / 2(d-1)) d /(d-1)} \mid 0 \in \mathcal{C}_{\infty}\right) \\
& \leq \mathbb{P}\left(\exists G_{n} \in \mathcal{G}_{n}, \exists i \in\left\{1, \ldots, m\left(G_{n}\right)\right\},\left|\partial^{o} L_{i}\right| \leq c_{3}^{\prime}\left|L_{i}\right|^{d /(d-1)} \mid 0 \in \mathcal{C}_{\infty}\right) \\
& \leq \frac{1}{\theta_{p}} \mathbb{P}\left(\begin{array}{c}
\text { There exists an open connected graph } G \text { contained in } \\
{\left[-n^{d}, n^{d}\right] \cap \mathbb{Z}^{d} \text { such that }|G| \geq t^{d /(d-1)},\left|\partial^{o} G\right| \leq c_{3}^{\prime}|G|^{(d-1) / d}}
\end{array}\right) \\
& \leq \frac{1}{\theta_{p}} \sum_{x \in\left[-n^{d}, n^{d}\right] \cap \mathbb{Z}^{d}} \mathbb{P}\left(\begin{array}{c}
\text { There exists an open connected graph } G \text { containing } \\
x \text { such that }|G| \geq t^{d /(d-1)},\left|\partial^{o} G\right| \leq c_{3}^{\prime}|G|^{(d-1) / d}
\end{array}\right) .
\end{aligned}
$$

Using the translation invariance together with inequality (5.2), we obtain

$$
\begin{aligned}
\mathbb{P}\left(\exists G_{n}\right. & \left.\in \mathcal{G}_{n}, \exists i \in\left\{1, \ldots, m\left(G_{n}\right)\right\},\left|\partial^{o} L_{i}\right| \leq c_{3}^{\prime} n^{(1-\varepsilon) d /(d-1)} \mid 0 \in \mathcal{C}_{\infty}\right) \\
& \leq \frac{\left(2 n^{d}\right)^{d}}{\theta_{p}} \mathbb{P}\left(\begin{array}{c}
\text { There exists an open connected graph } G \text { containing } 0 \\
\text { such that }|G| \geq t^{d /(d-1)},\left|\partial^{\circ} G\right| \leq c_{3}^{\prime}|G|^{(d-1) / d}
\end{array}\right) \\
& \leq \frac{\left(2 n^{d}\right)^{d}}{\theta_{p}} c_{1}^{\prime} \exp \left(-c_{2}^{\prime} n^{1-3 / 2 d}\right) .
\end{aligned}
$$

By construction, for all $i \in\left\{1, \ldots, m\left(G_{n}\right)\right\}$, we have $\partial^{\circ} L_{i} \subset \partial^{\circ} G_{n}$ and for all $j \in\left\{1, \ldots, m\left(G_{n}\right)\right\}$ such that $i \neq j$, we have $\partial^{o} L_{i} \cap \partial^{o} L_{j}=\emptyset$. Thus, with high probability,

$$
m\left(G_{n}\right) \leq \frac{\left|\partial^{o} G_{n}\right|}{c_{3}^{\prime} n^{(1-\varepsilon) d /(d-1)}} \leq \frac{C_{3}^{\prime} n^{d-1}}{c_{3}^{\prime} n^{1-3 / 2 d}} \leq \frac{C_{3}^{\prime}}{c_{3}^{\prime}} n^{d-2+3 / 2 d}
$$

Finally, by setting $c_{3}=C_{3}^{\prime} / c_{3}^{\prime}$, we obtain

$$
\begin{aligned}
\mathbb{P} & \left(\exists G_{n} \in \mathcal{G}_{n}, m\left(G_{n}\right)>c_{3} n^{d-2+3 / 2 d} \mid 0 \in \mathcal{C}_{\infty}\right) \\
& \leq \mathbb{P}\left(\varphi_{n} \geq c_{3}^{\prime} n^{-1} \mid 0 \in \mathcal{C}_{\infty}\right)+\mathbb{P}\left(\begin{array}{c}
\left.\exists G_{n} \in \mathcal{G}_{n}, \exists i \in\left\{1, \ldots, m\left(G_{n}\right)\right\}, \mid 0 \in \mathcal{C}_{\infty}\right) \\
\left|\partial^{o} L_{i}\right| \leq c_{3}^{\prime} n^{(1-\varepsilon) d /(d-1)}
\end{array}\right) \\
& \leq C_{1}^{\prime} \exp \left(-C_{2}^{\prime} n\right)+\frac{\left(2 n^{d}\right)^{d}}{\theta_{p}} c_{1}^{\prime} \exp \left(-c_{2}^{\prime} n^{1-3 / 2 d}\right) .
\end{aligned}
$$

This yields the result.

Using the control on the number of large components $m\left(G_{n}\right)$ of $\mathcal{C}_{\infty}$ enclosed in $\Gamma_{n}$ and a Peierls argument, we obtain the following control of $\left|\widetilde{\Gamma}_{n}\right|$ :

Proposition 5.7. Let $d \geq 2$ and $p>p_{c}(d)$. There exist positive constants $\beta_{0}, C_{1}$, $C_{2}$ depending only on $d$ and $p$ such that, for all $n \geq 1$, for all $\beta \geq \beta_{0}$,

$$
\mathbb{P}\left(\max _{G_{n} \in \mathcal{G}_{n}}\left|\widetilde{\Gamma}_{n}\right| \geq \beta n^{d-1} \mid 0 \in \mathcal{C}_{\infty}\right) \leq C_{1} \exp \left(-C_{2} n^{1-3 / 2 d}\right) .
$$

Proof: Let $k$ be a large integer that we will choose later. We consider a renormalization process of parameter $k$. Let $G_{n} \in \mathcal{G}_{n}$. First notice that as $\widetilde{\Gamma}_{n} \subset \bigcup_{B \in \widetilde{\Gamma}_{n}} B^{\prime}$, we have

$$
\left|\widetilde{\Gamma}_{n}\right| \leq(6 k)^{d}\left|\widetilde{\Gamma}_{n}\right|
$$


Thus, it is enough to control the quantity $\left|\widetilde{\Gamma}_{n}\right|$ to prove Proposition 5.7. We can rewrite $\widetilde{\Gamma}_{n}$ as

$$
\widetilde{\Gamma}_{n}=\bigcup_{i=1}^{m^{\prime}} \underline{A}_{i} \text { with } m^{\prime} \leq m\left(G_{n}\right)
$$

where the $\underline{A}_{i}$ are pairwise disjoint $*$-connected sets of cubes. Thanks to Theorem 1.4, there exist positive constants $C_{1}^{\prime}, C_{2}^{\prime}$ and $C_{3}^{\prime}$ depending only on $p$ and $d$ such that for all $n \geq 1$,

$$
\mathbb{P}\left(\varphi_{n} \geq C_{3}^{\prime} n^{-1} \mid 0 \in \mathcal{C}_{\infty}\right) \leq C_{1}^{\prime} \exp \left(-C_{2}^{\prime} n\right) .
$$

Let $G_{n} \in \mathcal{G}_{n}$. We have with probability at least $1-C_{1}^{\prime} \exp \left(-C_{2}^{\prime} n\right)$ that

$$
\left|\partial^{\circ} G_{n}\right| \leq C_{3}^{\prime} n^{d-1}
$$

We choose $\beta$ large enough such that

$$
C_{3}^{\prime} \leq \frac{\beta}{2 \cdot 4^{d}}
$$

so that

$$
\left|\partial^{o} G_{n}\right| \leq C_{3}^{\prime} n^{d-1} \leq \frac{\beta}{2 \cdot 4^{d}} n^{d-1} .
$$

We now want to sum over the possible realizations of $\widetilde{\Gamma}_{n}$. Using Proposition 5.6 together with inequality (5.3), we get

$$
\begin{aligned}
& \mathbb{P}\left(\exists G_{n} \in \mathcal{G}_{n},\left|\widetilde{\Gamma}_{n}\right| \geq \beta n^{d-1} \mid 0 \in \mathcal{C}_{\infty}\right) \\
& \leq \mathbb{P}\left(\exists G_{n} \in \mathcal{G}_{n}, \begin{array}{c}
\sum_{i=1}^{m^{\prime}}\left|\underline{A}_{i}\right| \geq \beta n^{d-1}, m^{\prime} \leq c_{3}^{\prime} n^{d-2+3 / 2 d}, \\
\left|\partial^{o} G_{n}\right| \leq \frac{\beta}{2 \cdot 4^{d}} n^{d-1}
\end{array} \mid 0 \in \mathcal{C}_{\infty}\right) \\
& +c_{1} \exp \left(-c_{2} n^{1-3 / 2 d}\right)+C_{1}^{\prime} \exp \left(-C_{2}^{\prime} n\right) \\
& \leq \sum_{j \geq \beta n^{d-1}} \sum_{m^{\prime}=1}^{c_{3}^{\prime} n^{d-2+3 / 2 d}} \sum_{\substack{j_{1}+\ldots+j_{m^{\prime}}=j \\
j_{1}>0, \ldots, j_{m^{\prime}}>0}} \sum_{x_{1}, \ldots, x_{m^{\prime}} \in\left[-n^{d}, n^{d}\right]^{d}} \sum_{\substack{A_{1} \in \text { Animals }_{x_{1}} \\
\left|A_{1}\right|=j_{1}}} \ldots \\
& \ldots \sum_{\substack{A_{m^{\prime}} \in \text { Animals }_{x_{m^{\prime}}} \\
\left|A_{m^{\prime}}\right|=j_{m^{\prime}}}} \mathbb{P}\left(\exists G_{n} \in \mathcal{G}_{n}, \underline{\widetilde{\Gamma}}_{n}=\bigcup_{i=1}^{m^{\prime}} A_{i},\left|\partial^{o} G_{n}\right| \leq \frac{\beta}{2 \cdot 4^{d}} n^{d-1} \mid 0 \in \mathcal{C}_{\infty}\right) \\
& +c_{1} \exp \left(-c_{2} n^{1-3 / 2 d}\right)+C_{1}^{\prime} \exp \left(-C_{2}^{\prime} n\right) \text {. }
\end{aligned}
$$

Let us assume $\widetilde{\Gamma}_{n}=\bigcup_{i=1}^{m^{\prime}} A_{i}$. We can extract from $\widetilde{\Gamma}_{n}$ a set of $k$-cubes $\widetilde{\widetilde{\Gamma}}_{n}^{\prime}$ such that $\left|\widetilde{\Gamma}_{n}^{\prime}\right| \geq\left|\widetilde{\Gamma}_{n}\right| / 4^{d}$ and for any $\mathbf{i} \neq \mathbf{j}$ such that $B_{k}(\mathbf{i}), B_{k}(\mathbf{j}) \in{\widetilde{\Gamma^{\prime}}}_{n}{ }_{n}$ we have $B_{k}^{\prime}(\mathbf{i}) \cap B_{k}^{\prime}(\mathbf{j})=\emptyset$. As the rare event depends only on the configuration in the $3 k$-cube $B_{k}^{\prime}(\mathbf{j})$, the two following events $\left\{\right.$ a rare event occurs in $\left.B_{k}(\mathbf{i})\right\}$ and $\left\{\right.$ a rare event occurs in $\left.B_{k}(\mathbf{j})\right\}$ are independent. Using Proposition 5.4, we obtain

$$
\mathbb{P}\left(\exists G_{n} \in \mathcal{G}_{n}, \widetilde{\Gamma}_{n}=\bigcup_{i=1}^{m^{\prime}} A_{i},\left|\partial^{o} G_{n}\right| \leq \frac{\beta}{2 \cdot 4^{d}} n^{d-1} \mid 0 \in \mathcal{C}_{\infty}\right)
$$




$$
\begin{aligned}
& \leq \mathbb{P}\left(\exists G_{n} \in \mathcal{G}_{n}, \widetilde{\widetilde{\Gamma}}_{n}=\bigcup_{i=1}^{m^{\prime}} A_{i},\left|\underline{\widetilde{\Gamma}}_{n}\right| \geq j / 4^{d},\left|\partial^{o} G_{n}\right| \leq \frac{\beta}{2 \cdot 4^{d}} n^{d-1} \mid 0 \in \mathcal{C}_{\infty}\right) \\
& \leq \mathbb{P}\left(\exists G_{n} \in \mathcal{G}_{n}, \quad \begin{array}{c}
\widetilde{\widetilde{\Gamma}}_{n}=\bigcup_{i=1}^{m^{\prime}} A_{i},\left|\partial^{o} G_{n}\right| \leq \frac{\beta}{2 \cdot 4^{d}} n^{d-1}, \\
\mid\left\{B \subset{\underline{\Gamma_{n}^{\prime}}}_{n}, B \text { abnormal }\right\}\left|\geq j / 4^{d}-\right| \partial^{o} G_{n} \mid
\end{array} \mid 0 \in \mathcal{C}_{\infty}\right) \\
& \leq \mathbb{P}\left(\exists G_{n} \in \mathcal{G}_{n}, \widetilde{\Gamma}_{n}=\bigcup_{i=1}^{m^{\prime}} A_{i}, \mid\left\{B \subset \underline{\widetilde{\Gamma}}_{n}^{\prime}, B \text { abnormal }\right\} \mid \geq j /\left(2.4^{d}\right)\right) \cdot \frac{1}{\theta_{p}} \\
& \leq \frac{4^{d}}{\theta_{p}} \sum_{l \geq j /\left(2.4^{d}\right)}\left(C_{1} \mathrm{e}^{-C_{2} k}\right)^{l} \\
& \leq \frac{2 \cdot 4^{d}}{\theta_{p}}\left(C_{1} \mathrm{e}^{-C_{2} k}\right)^{j /\left(2.4^{d}\right)}
\end{aligned}
$$

where $k$ will be chosen large enough such that $C_{1} \mathrm{e}^{-C_{2} k} \leq 1 / 2$. So together with inequality (5.4) and using Lemma 2.1, we obtain

$$
\begin{aligned}
& \mathbb{P}\left(\exists G_{n} \in \mathcal{G}_{n},\left|\widetilde{\Gamma}_{n}\right| \geq \beta n^{d-1} \mid 0 \in \mathcal{C}_{\infty}\right) \\
& \leq \sum_{j \geq \beta n^{d-1}} \sum_{m^{\prime}=1}^{c_{3}^{\prime} n^{d-2+3 / 2 d}} \sum_{\substack{j_{1}+\cdots+j_{m^{\prime}}=j \\
j_{1}>0, \ldots, j_{m^{\prime}}>0}} \sum_{x_{1}, \ldots, x_{m^{\prime}} \in\left[-n^{d}, n^{d}\right]^{d}} \sum_{\substack{A_{1} \in \text { Animals }_{x_{1}} \\
\left|A_{1}\right|=j_{1}}} \ldots \\
& \cdots \sum_{\substack{A_{m^{\prime}} \in \text { Animals }_{x_{m}} \\
\left|A_{m^{\prime}}\right|=j_{m^{\prime}}}} \frac{2 \cdot 4^{d}}{\theta_{p}}\left(C_{1} \mathrm{e}^{-C_{2} k}\right)^{j /\left(2.4^{d}\right)}+c_{1} \mathrm{e}^{-c_{2} n^{1-3 / 2 d}}+C_{1}^{\prime} \mathrm{e}^{-C_{2}^{\prime} n} \\
& \leq \frac{2 \cdot 4^{d}}{\theta_{p}} \sum_{j \geq \beta n^{d-1}}\left(C_{1} \mathrm{e}^{-C_{2} k}\right)^{\frac{j}{2.4^{d}}} \sum_{m^{\prime}=1}^{c_{3}^{\prime} n^{d-2+3 / 2 d}} \sum_{\substack{j_{1}+\cdots+j_{m^{\prime}}=j \\
j_{1}>0, \ldots, j_{m^{\prime}}>0}}(2 n)^{d^{2} m^{\prime}} 7^{d j_{1}} \cdots 7^{d j_{m^{\prime}}} \\
& +c_{1} \mathrm{e}^{-c_{2} n^{1-3 / 2 d}}+C_{1}^{\prime} \mathrm{e}^{-C_{2}^{\prime} n} \\
& \leq \frac{2 \cdot 4^{d}}{\theta_{p}} \sum_{j \geq \beta n^{d-1}} 7^{d j}\left(C_{1} \mathrm{e}^{-C_{2} k}\right)^{j /\left(2.4^{d}\right)} \sum_{m^{\prime}=1}^{c_{3}^{\prime} n^{d-2+3 / 2 d}}\left(2 n^{d}\right)^{d m^{\prime}} \text {. } \\
& \times\left|\left\{\left(j_{1}, \ldots, j_{m^{\prime}}\right): \begin{array}{l}
j_{1}+\cdots+j_{m^{\prime}}=j, \\
j_{1}>0, \ldots, j_{m^{\prime}}>0
\end{array}\right\}\right|+c_{1} \mathrm{e}^{-c_{2} n^{1-3 / 2 d}}+C_{1}^{\prime} \mathrm{e}^{-C_{2}^{\prime} n} \\
& \leq \frac{2 \cdot 4^{d}}{\theta_{p}}\left(2 n^{d}\right)^{d\left(c_{3}^{\prime} n^{d-2+3 / 2 d}+2\right)} \sum_{j \geq \beta n^{d-1}}\left(2 \cdot 7^{d}\right)^{j}\left(C_{1} \mathrm{e}^{-C_{2} k}\right)^{j /\left(2.4^{d}\right)} \\
& +c_{1} \mathrm{e}^{-c_{2} n^{1-3 / 2 d}}+C_{1}^{\prime} \mathrm{e}^{-C_{2}^{\prime} n}
\end{aligned}
$$

We now choose $k$ large enough such that

$$
C_{1} \mathrm{e}^{-C_{2} k} \leq \frac{1}{2} \quad \text { and } \quad\left(\left(2 \cdot 7^{d}\right)^{2 \cdot 4^{d}} C_{1} \mathrm{e}^{-C_{2} k}\right)^{1 /\left(2.4^{d}\right)} \leq \mathrm{e}^{-1}
$$

Finally, we get

$$
\mathbb{P}\left(\exists G_{n} \in \mathcal{G}_{n},\left|\widetilde{\Gamma}_{n}\right| \geq \beta n^{d-1} \mid 0 \in \mathcal{C}_{\infty}\right)
$$




$$
\begin{aligned}
& \leq \frac{2\left(2 n^{d}\right)^{d\left(c_{3}^{\prime} n^{d-2+3 / 2 d}+2\right)} 4^{d}}{\theta_{p}} \sum_{j \geq \beta n^{d-1}}\left(\left(2 \cdot 7^{d}\right)^{2 \cdot 4^{d}} C_{1} \mathrm{e}^{-C_{2} k}\right)^{j /\left(2.4^{d}\right)} \\
& \quad+c_{1} \mathrm{e}^{-c_{2} n^{1-3 / 2 d}}+C_{1}^{\prime} \mathrm{e}^{-C_{2}^{\prime} n} \\
& \leq \frac{4^{d+1} \exp \left(2 d^{2} c_{3}^{\prime} n^{d-2+3 / 2 d} \log n-\beta n^{d-1}\right)}{\theta_{p}}+c_{1} \mathrm{e}^{-c_{2} n^{1-3 / 2 d}}+C_{1}^{\prime} \mathrm{e}^{-C_{2}^{\prime} n} .
\end{aligned}
$$

This yields the result for $\beta \geq \beta_{0}$ where $\beta_{0}$ is such that for all $n \geq 0$, we have $\beta_{0}>\left(4 d^{2} c_{3}^{\prime} \log n\right) / n^{1-3 / 2 d}$.

We can now build the relevant continuous object $P_{n}$. Given a finite set of edges $S$, we define

$$
\operatorname{hull}(S)=\left\{x \in \mathbb{Z}^{d}: \text { any path from } x \text { to infinity has to use an edge of } S\right\}
$$

and

$$
H_{n}=\operatorname{hull}\left(\Gamma_{n}\right) \backslash\left(\bigcup_{i=1}^{m} \operatorname{hull}\left(\widehat{\Gamma}_{n}^{(i)}\right)\right) .
$$

We define $P_{n}$ and its associated measure $\nu_{n}$ as

$$
\begin{gathered}
P_{n}=\frac{1}{n}\left(H_{n}+\left[-\frac{1}{2}, \frac{1}{2}\right]^{d}\right), \\
\forall E \in \mathcal{B}\left(\mathbb{R}^{d}\right), \nu_{n}(E)=\theta_{p} \mathcal{L}^{d}\left(P_{n} \cap E\right) .
\end{gathered}
$$

We obtain a control on the size of the perimeter of $P_{n}$ by a straightforward application of Proposition 5.7:

Corollary 5.8. Let $d \geq 2$ and $p>p_{c}(d)$. There exist positive constants $\beta_{0}, C_{1}$, $C_{2}$ depending only on $d$ and $p$ such that for all $n \geq 1$, for all $\beta>\beta_{0}$,

$$
\mathbb{P}\left(\max _{G_{n} \in \mathcal{G}_{n}} \mathcal{P}\left(n P_{n}\right) \geq \beta n^{d-1} \mid 0 \in \mathcal{C}_{\infty}\right) \leq C_{1} \mathrm{e}^{-c_{2} n^{1-3 / 2 d}} .
$$

The following Lemma will be useful to compare the measure $\nu_{n}$ with the measure associated to $F_{n}$.

Lemma 5.9. Let $G_{n} \in \mathcal{G}_{n}$ and $F_{n}$ as defined in (5.1). We have $F_{n}=H_{n} \cap \mathcal{C}_{\infty}$.

Proof: Let $G_{n} \in \mathcal{G}_{n}$. Let $x \in H_{n} \cap \mathcal{C}_{\infty}$, then $x$ belongs to $\mathcal{C}_{\infty} \cap$ hull $\Gamma_{n}$ but is not in any of the large connected components $L_{1}, \ldots, L_{m}$. Therefore, $x$ belongs to $G_{n}$ or to one of the small components $S_{1}, \ldots, S_{N}$ and so $x \in F_{n}$.

Conversely, let $x \in F_{n}$. It is clear that $x \in \operatorname{hull}\left(\Gamma_{n}\right)$. Let us assume $x \in G_{n}$ and that there exists $i$ such that $x \in \operatorname{hull}\left(\widehat{\Gamma}_{n}^{(i)}\right)$. As $G_{n}$ is connected there exists an open path $\gamma$ in $G_{n}$ that joins $x$ with $G_{n} \backslash \widehat{\Gamma}_{n}^{(i)}$. As the edges of $\widehat{\Gamma}_{n}^{(i)} \backslash \partial^{o} L_{i}$ are closed, $\gamma$ must use an edge of $\partial^{\circ} L_{i}$ and so go through a vertex of $L_{i}$. That is a contradiction as the path $\gamma$ uses only vertices in $G_{n}$. Let us now assume that $x \in S_{j}$ and $x \in \operatorname{hull}\left(\widehat{\Gamma}_{n}^{(i)}\right)$ for some $i$ and $j$. As $x \in \mathcal{C}_{\infty}, x$ is connected to infinity by an open path $\gamma^{\prime}$. However, by the same arguments, to exit hull $\left(\widehat{\Gamma}_{n}^{(i)}\right)$, the path $\gamma^{\prime}$ has to go through a vertex of $L_{i}$. Thus, there exist an open path in $\mathcal{C}_{\infty} \backslash G_{n}$ that joins $x$ to $L_{i}$. That is a contradiction as $x \notin L_{i}$.

Finally, $F_{n} \subset H_{n} \cap \mathcal{C}_{\infty}$. 
5.3. Closeness of measures. We shall show that for any ball of constant radius centered at a point $x \in \mathbb{Z}^{d}$, the measures $\nu_{n}$ and $\mu_{n}$ restricted to this ball are close to each other in some weak sense.

Proposition 5.10. Let $p>p_{c}(d)$ and $r>0$. Let $\left.u:\right] 0,+\infty[\rightarrow] 0,+\infty[$ be a nondecreasing function such that $\lim _{t \rightarrow 0} u(t)=0$. For all $\delta>0$, there exist $C_{1}$ and $C_{2}$ depending on $d, p, u$ and $\delta$ such that for all $n \geq 1$, for any finite set $\mathfrak{F}_{n}$ of uniformly continuous functions that satisfies:

$$
\forall f \in \mathfrak{F}_{n} \quad\|f\|_{\infty} \leq 1 \quad \text { and } \quad \forall x, y \in \mathbb{R}^{d} \quad|f(x)-f(y)| \leq u\left(\|x-y\|_{2}\right),
$$

we have

$$
\mathbb{P}\left(\max _{G_{n} \in \mathcal{G}_{n}} \sup _{f \in \mathfrak{F}}\left|\mu_{n}\left(f \mathbb{1}_{B(x, r)}\right)-\nu_{n}\left(f \mathbb{1}_{B(x, r)}\right)\right|>\delta \mid 0 \in \mathcal{C}_{\infty}\right) \leq C_{1} \mathrm{e}^{-C_{2} n^{1-3 / 2 d}} .
$$

Remark 5.11. We state here the result in a general form. In the following, we will apply this Proposition for the particular case of sets of functions that are translates of the same function. The function $u$ is an upper bound on the modulus of continuity of the functions in $\mathfrak{F}_{n}$. If we think of $\mathfrak{F}_{n}$ as a set that grows with $n$, this condition may be interpreted as a sufficient condition to obtain compactness for the set $\mathfrak{F}_{n}$ in the limit.

To prove this result, we will need the following proposition that is a corollary of the results in Pisztora (1996):

Proposition 5.12. Let $d \geq 2$ and $p>p_{c}(d)$. Let $r>0$, and let $Q \subset \mathbb{R}^{d}$ be a cube of side length $2 r$. Let $\delta>0$. There exist positive constants $c_{1}$ and $c_{2}$ depending on $d, p$ and $\delta$ such that

$$
\mathbb{P}\left(\frac{\left|\mathcal{C}_{\infty} \cap Q\right|}{\mathcal{L}^{d}(Q)} \notin\left(\theta_{p}-\delta, \theta_{p}+\delta\right)\right) \leq c_{1} \exp \left(-c_{2} r^{d-1}\right) .
$$

Proof of Proposition 5.10: Let $\delta>0$ and $\varepsilon>0$ that we will choose later. Let $u:] 0,+\infty[\rightarrow] 0,+\infty\left[\right.$ be a non-decreasing function such that $\lim _{t \rightarrow 0} u(t)=0$. Let $n \geq 1$. Let $\mathfrak{F}_{n}$ be a finite set of uniformly continuous function that satisfies:

$$
\forall f \in \mathfrak{F}_{n} \quad\|f\|_{\infty} \leq 1 \quad \text { and } \quad \forall x, y \in \mathbb{R}^{d} \quad|f(x)-f(y)| \leq u\left(\|x-y\|_{2}\right),
$$

We define

$$
\widetilde{\mu}_{n}=\frac{1}{n^{d}} \sum_{x \in V\left(F_{n}\right)} \delta_{x / n} .
$$

Thanks to Theorem 1.4, there exists a constant $\eta_{3}$ depending only on the dimension such that

$$
\mathbb{P}\left(n \varphi_{n} \geq \eta_{3} \mid 0 \in \mathcal{C}_{\infty}\right) \leq C_{1} \exp \left(-C_{2} n\right)
$$

Let $G_{n} \in \mathcal{G}_{n}$, with probability at least $1-C_{1} \exp \left(-C_{2} n\right)$, we have

$$
\frac{n\left|\partial^{\circ} G_{n}\right|}{\left|G_{n}\right|} \leq \eta_{3}
$$

and so $\left|\partial^{o} G_{n}\right| \leq \eta_{3} n^{d-1}$. As each small component $S_{j}$ is such that $\partial^{o} S_{j} \cap \partial^{o} G_{n} \neq \emptyset$, the number $N$ of small components is at most $\eta_{3} n^{d-1}$ and by definition of $F_{n}$,

$$
\left|F_{n} \backslash G_{n}\right| \leq \sum_{j=1}^{N}\left|S_{j}\right| \leq \eta_{3} n^{d-1 / 2(d-1)}
$$


Finally, with probability at least $1-C_{1} \exp \left(-C_{2} n\right)$, for all $f \in \mathfrak{F}_{n}$,

$$
\left|\mu_{n}(f)-\widetilde{\mu}_{n}(f)\right| \leq \frac{1}{n^{d}}\|f\|_{\infty}\left|F_{n} \backslash G_{n}\right| \leq \eta_{3} n^{-1 / 2(d-1)},
$$

and

$$
\mathbb{P}\left(\max _{G_{n} \in \mathcal{G}_{n}} \sup _{f \in \mathfrak{F}_{n}}\left|\mu_{n}\left(f \mathbb{1}_{B(x, r)}\right)-\nu_{n}\left(f \mathbb{1}_{B(x, r)}\right)\right|>\eta_{3} n^{-1 / 2(d-1)}\right) \leq C_{1} \mathrm{e}^{-C_{2} n}
$$

where $\mathbb{P}$ represents the probability measure conditioned on the event $\left\{0 \in \mathcal{C}_{\infty}\right\}$. Let $x \in \mathbb{R}^{d}$ and let $r>0$. Let $f \in \mathfrak{F}_{n}$. We now would like to estimate the quantity

$$
\left|\widetilde{\mu}_{n}\left(f \mathbb{1}_{B(x, r)}\right)-\nu_{n}\left(f \mathbb{1}_{B(x, r)}\right)\right| .
$$

We adapt the proof of 16.2 in Cerf (2006). We use again a renormalization argument but at a different scale $L=K \ln n$. We consider the lattice rescaled by this factor $L$. We say that a cluster $C$ is crossing in a box $B$ if for any two opposite faces of $B$, the cluster $C$ contains an open path in $B$ that joins these two faces. Let $\varepsilon>0$. For $\underline{y} \in \mathbb{Z}^{d}$, we define $B_{n}(\underline{y})=(2 L \underline{y} / n)+[-L / n, L / n]^{d}$ and $B_{n}^{\prime}(\underline{y})=$ $(2 L \underline{y} / n)+[-3 L / n, 3 L / n]^{d}$. Let $X(\underline{y})$ be the indicator function of the event $\mathcal{E}_{n}(\underline{y})$. This event occurs if

- Inside $n B_{n}^{\prime}(y)$, there is a unique crossing cluster $C^{\prime}$ that crosses the $3^{d}$ sub-boxes of $n B_{n}^{\prime}(\underline{y})$. Moreover, $C^{\prime}$ is the only cluster in $n B_{n}^{\prime}(\underline{y})$ of diameter larger than $L$.

- Inside $n B_{n}(\underline{y})$, there is a crossing cluster $C^{*}$ such that

$$
\left|C^{*}\right| \geq\left(\theta_{p}-\varepsilon\right) \mathcal{L}^{d}\left(n B_{n}(\underline{y})\right) .
$$

- We have $\mid\left\{x \in n B_{n}(\underline{y}): x \longleftrightarrow \partial n B_{n}(\underline{y}\} \mid \leq\left(\theta_{p}+\varepsilon\right) \mathcal{L}^{d}\left(n B_{n}(\underline{y})\right)\right.$

On the event $\mathcal{E}_{n}(y)$, any cluster $C \subset n B_{n}(y)$ that is connected by an open path to $\partial\left(n B_{n}^{\prime}(\underline{y})\right)$ is the unique crossing cluster, i.e., $C=C^{\prime}=C^{*}$ and so it also satisfies

$$
\frac{|C|}{\mathcal{L}^{d}\left(n B_{n}(\underline{y})\right)} \in\left[\theta_{p}-\varepsilon, \theta_{p}+\varepsilon\right] .
$$

The family $(X(\underline{y}))_{\underline{y} \in \mathbb{Z}^{d}}$ is a site percolation process on the macroscopic lattice. The states of the sites are not independent from each other but there is only a short range dependency. Indeed, for any $\underline{y}$ and $\underline{z}$ such that $|\underline{y}-\underline{z}|_{\infty} \geq 3$, we have that $X(\underline{y})$ and $X(\underline{z})$ are independent. We define the connected component $\mathcal{C}(\underline{y})$ of $\underline{y}$ as

$$
\mathcal{C}(\underline{y})=\left\{\underline{z} \in \mathbb{Z}^{d}: \underline{z} \text { is connected to } \underline{y} \text { by a macroscopic open path }\right\} .
$$

Let

We have

$$
\underline{D}=\left\{\underline{y} \in \mathbb{Z}^{d}: B_{n}(\underline{y}) \subset B(x, r)\right\} .
$$

$$
|\underline{D}| L^{d} \leq n^{d} \mathcal{L}^{d}(B(x, r)) .
$$

There exists an integer $n_{0}=n_{0}(u(\varepsilon))$ such that, for $n \geq n_{0}(u(\varepsilon))$, we have $L / n \leq$ $u(\varepsilon)$ so that

$$
\begin{gathered}
\mathcal{L}^{d}\left(B(x, r) \backslash \bigcup_{\underline{y} \in \underline{D}} B_{n}(\underline{y})\right) \leq \varepsilon \mathcal{L}^{d}(B(x, r)), \\
\forall w, z \in \mathbb{R}^{d},\|w-z\|_{2} \leq \frac{L}{n} \Rightarrow|f(x)-f(y)| \leq \varepsilon .
\end{gathered}
$$


The last statement comes from the fact that $f$ belongs to $\mathfrak{F}_{n}$. By decomposing $\left|\widetilde{\mu}_{n}\left(f \mathbb{1}_{B(x, r)}\right)-\nu_{n}\left(f \mathbb{1}_{B(x, r)}\right)\right|$ on cubes of size $L / n$, we obtain:

$$
\begin{aligned}
& \left|\widetilde{\mu}_{n}\left(f \mathbb{1}_{B(x, r)}\right)-\nu_{n}\left(f \mathbb{1}_{B(x, r)}\right)\right| \\
& \quad \leq 2 \mathcal{L}^{d}\left(B(x, r) \backslash \bigcup_{\underline{y} \in \underline{D}} B_{n}(\underline{y})\right)+\sum_{\underline{y} \in \underline{D}}\left|\int_{B_{n}(\underline{y})} f d \widetilde{\mu}_{n}-\int_{B_{n}(\underline{y})} f d \nu_{n}\right| \\
& \quad \leq 4 \varepsilon \mathcal{L}^{d}(B(x, r))+\sum_{\underline{y} \in \underline{D}}\left|\widetilde{\mu}_{n}\left(B_{n}(\underline{y})\right)-\nu_{n}\left(B_{n}(\underline{y})\right)\right| .
\end{aligned}
$$

Let $y \in \underline{D}$. We need to distinguish several cases:

- If $B_{n}(\underline{y}) \cap P_{n}=\emptyset$, then $\nu_{n}\left(B_{n}(\underline{y})\right)=\widetilde{\mu}_{n}\left(B_{n}(\underline{y})\right)=0$. From now on we will only consider cubes such that $B_{n}(y) \cap P_{n} \neq \emptyset$.

- If $B_{n}(\underline{y}) \not \subset P_{n}$, then we bound

$$
\left|\widetilde{\mu}_{n}\left(B_{n}(\underline{y})\right)-\nu_{n}\left(B_{n}(\underline{y})\right)\right| \leq \frac{1}{n^{d}}\left|B_{n}(\underline{y})\right|
$$

and as $B_{n}(\underline{y}) \cap P_{n} \neq \emptyset$, the cube intersects the boundary of $P_{n}$. Thus,

$$
B_{n}(\underline{y}) \subset\left\{z \in \mathbb{R}^{d}: d_{\infty}\left(z, \partial P_{n} \cap B(x, r)\right) \leq \frac{L}{n}\right\} .
$$

Moreover,

$$
\begin{aligned}
\mathcal{L}^{d} & \left(\left\{z \in \mathbb{R}^{d}: d_{\infty}\left(z, \partial P_{n} \cap B(x, r)\right) \leq \frac{L}{n}\right\}\right) \\
& \leq\left|\left\{x \in H_{n}, \exists y \in \mathbb{Z}^{d} \backslash H_{n},\|x-y\|_{1}=1\right\} \cap B(n x, n r+d)\right|\left(\frac{2 L+2}{n}\right)^{d} \\
& \leq \mathcal{P}\left(n P_{n}, B(n x, n r+d)\right)\left(\frac{3 L}{n}\right)^{d} \\
& \leq \mathcal{P}\left(P_{n}, B(x, r+d)\right) \frac{(3 L)^{d}}{n} .
\end{aligned}
$$

- If $B_{n}(\underline{y}) \subset P_{n}$ and $|\mathcal{C}(\underline{y})|=\infty$, then the crossing cluster $C^{*}$ of $B_{n}(\underline{y})$ is a portion of $\mathcal{C}_{\infty}$ and

$$
\nu_{n}\left(B_{n}(\underline{y})\right)=\theta_{p} \frac{\mathcal{L}^{d}\left(n B_{n}(\underline{y})\right)}{n^{d}} \text { and } \widetilde{\mu}_{n}\left(B_{n}(\underline{y})\right)=\frac{\left|\left(n B_{n}(\underline{y})\right) \cap C^{*}\right|}{n^{d}} .
$$

Thus, we have

$$
\widetilde{\mu}_{n}\left(B_{n}(\underline{y})\right) \in\left[\left(\theta_{p}-\varepsilon\right) \mathcal{L}^{d}\left(B_{n}(\underline{y})\right),\left(\theta_{p}+\varepsilon\right) \mathcal{L}^{d}\left(B_{n}(\underline{y})\right)\right]
$$

and

$$
\left|\widetilde{\mu}_{n}\left(B_{n}(\underline{y})\right)-\nu_{n}\left(B_{n}(\underline{y})\right)\right| \leq \varepsilon \mathcal{L}^{d}\left(B_{n}(\underline{y})\right) .
$$

- If $B_{n}(\underline{y}) \subset P_{n}$ and $|\mathcal{C}(\underline{y})|<\infty$, then we bound

$$
\left|\widetilde{\mu}_{n}\left(B_{n}(\underline{y})\right)-\nu_{n}\left(B_{n}(\underline{y})\right)\right| \leq \mathcal{L}^{d}\left(B_{n}(\underline{y})\right) \mathbb{1}_{|\mathcal{C}(\underline{y})|<\infty}
$$

By summing the previous inequalities over $\underline{y} \in \underline{D}$, thanks to inequality (5.6) and (5.7), we obtain

$$
\left|\widetilde{\mu}_{n}\left(f \mathbb{1}_{B(x, r)}\right)-\nu_{n}\left(f \mathbb{1}_{B(x, r)}\right)\right|
$$




$$
\leq \mathcal{L}^{d}(B(x, r))\left(5 \varepsilon+\frac{1}{|\underline{D}|} \sum_{\underline{y} \in \underline{D}} \mathbb{1}_{|\mathcal{C}(\underline{y})|<\infty}\right)+\mathcal{P}\left(P_{n}, B(x, r+d)\right) \frac{(3 L)^{d}}{n} .
$$

Let $c(r)=6 \mathcal{L}^{d}(B(0, r))+3^{d}$, we get

$$
\begin{aligned}
& \underline{\mathbb{P}}\left(\max _{G_{n} \in \mathcal{G}_{n}} \sup _{f \in \widetilde{F}_{n}}\left|\widetilde{\mu}_{n}\left(f \mathbb{1}_{B(x, r)}\right)-\nu_{n}\left(f \mathbb{1}_{B(x, r)}\right)\right|>c(r) \varepsilon\right) \\
& \quad \leq \frac{1}{\theta_{p}} \mathbb{P}\left(\frac{1}{|\underline{D}|} \sum_{\underline{y} \in \underline{D}} \mathbb{1}_{|\mathcal{C}(\underline{y})|<\infty} \geq \varepsilon\right)+\underline{\mathbb{P}}\left(\max _{G_{n} \in \mathcal{G}_{n}} \mathcal{P}\left(P_{n}, B(x, r+d)\right) \geq \varepsilon \frac{n}{L^{d}}\right) .
\end{aligned}
$$

Besides, using Corollary 5.8, for $n$ large enough, we obtain

$$
\underline{\mathbb{P}}\left(\max _{G_{n} \in \mathcal{G}_{n}} \mathcal{P}\left(P_{n}, B(x, r+d)\right) \geq \varepsilon \frac{n}{L^{d}}\right) \leq \mathbb{P}\left(\max _{G_{n} \in \mathcal{G}_{n}} \mathcal{P}\left(P_{n}\right) \geq \beta\right) \leq c_{1} \mathrm{e}^{-c_{2} n^{1-3 / 2 d}} .
$$

Let $\Lambda$ be the cube centered at $x$ of side length $2 r$. We define

$$
\underline{\Lambda}=\left\{\underline{y} \in \mathbb{Z}^{d}: B_{n}(\underline{y}) \subset \Lambda\right\} .
$$

As $B(x, r) \subset \Lambda$, we have $\underline{D} \subset \underline{\Lambda}$ and

$$
\frac{1}{|\underline{D}|} \sum_{\underline{y} \in \underline{D}} \mathbb{1}_{|\mathcal{C}(\underline{y})|<\infty} \leq \frac{(2 d)^{d}}{|\underline{\Lambda}|} \sum_{\underline{y} \in \underline{\Lambda}} \mathbb{1}_{|\mathcal{C}(\underline{y})|<\infty} .
$$

Let $q \in[0,1]$ be such that $\theta_{q}>1-\varepsilon /\left(2(2 d)^{d}\right)$. As the family $(X(y))_{y \in \mathbb{Z}^{d}}$ is identically distributed, has a short range dependency and is such that $\mathbb{P}(\bar{X}(\underline{\overline{0}})=1)$ goes to 1 when $n$ goes to infinity (see for instance Chapter 9 in Cerf, 2006), then we can apply Liggett, Schonmann and Stacey's result (Liggett et al., 1997): for $n$ large enough, the family $\left(X(\underline{y}), \underline{y} \in \mathbb{Z}^{d}\right)$ stochastically dominates $\left(\tilde{X}(\underline{y}), \underline{y} \in \mathbb{Z}^{d}\right)$ a family of independent Bernoulli variable of parameter $q$. We denote by $\widetilde{\mathcal{C}}_{\infty}$ the unique infinite cluster of the Bernoulli field $(\widetilde{X}(\underline{y}))_{\underline{y} \in \mathbb{Z}^{d}}$. Using inequality (5.10) and the stochastic domination, we get

$$
\begin{aligned}
\mathbb{P}\left(\frac{1}{|\underline{D}|} \sum_{\underline{y} \in \underline{D}} \mathbb{1}_{|\mathcal{C}(\underline{y})|<\infty} \geq \varepsilon\right) & \leq \mathbb{P}\left(\frac{(2 d)^{d}}{|\underline{\Lambda}|} \sum_{\underline{y} \in \underline{\Lambda}} \mathbb{1}_{|\mathcal{C}(\underline{y})|<\infty} \geq \varepsilon\right) \\
& \leq \mathbb{P}\left(\frac{1}{|\underline{\Lambda}|} \sum_{\underline{y} \in \underline{\Lambda}} \mathbb{1}_{\underline{y} \notin \tilde{\mathcal{C}}_{\infty}} \geq \frac{\varepsilon}{(2 d)^{d}}\right) \\
& \leq \mathbb{P}\left(\frac{\left|\underline{\Lambda} \cap \underline{\widetilde{\mathcal{C}}}_{\infty}\right|}{|\underline{\Lambda}|} \notin\left(\theta_{q}-\frac{\varepsilon}{2(2 d)^{d}}, \theta_{q}+\frac{\varepsilon}{2(2 d)^{d}}\right)\right) .
\end{aligned}
$$

Using Proposition 5.12, we obtain

$$
\mathbb{P}\left(\frac{1}{|\underline{D}|} \sum_{\underline{y} \in \underline{D}} \mathbb{1}_{|\mathcal{C}(\underline{y})|<\infty} \geq \varepsilon\right) \leq c_{1}^{\prime} \exp \left(-c_{2}^{\prime}\left(\frac{r n}{L}\right)^{d-1}\right) .
$$


We set $\varepsilon=\delta /(2 c(r))$. Finally, thanks to inequalities (5.5), (5.8), (5.9) and (5.11), we have for $n \geq n_{0}(u(\varepsilon))$

$$
\begin{aligned}
\underline{\mathbb{P}}\left(\max _{G_{n} \in \mathcal{G}_{n}} \sup _{f \in \widetilde{F}_{n}}\left|\widetilde{\mu}_{n}\left(f \mathbb{1}_{B(x, r)}\right)-\nu_{n}\left(f \mathbb{1}_{B(x, r)}\right)\right|>\delta\right) \\
\leq \underline{\mathbb{P}}\left(\max _{G_{n} \in \mathcal{G}_{n}} \sup _{f \in \widetilde{F}_{n}}\left|\mu_{n}(f)-\widetilde{\mu}_{n}(f)\right|>\delta / 2\right) \\
\quad+\underline{\mathbb{P}}\left(\max _{G_{n} \in \mathcal{G}_{n}} \sup _{f \in \widetilde{F}_{n}}\left|\widetilde{\mu}_{n}\left(f \mathbb{1}_{B(x, r)}\right)-\nu_{n}\left(f \mathbb{1}_{B(x, r)}\right)\right|>c(r) \varepsilon\right) \\
\leq C_{1} \exp \left(-C_{2} n\right)+\frac{c_{1}^{\prime}}{\theta_{p}} \exp \left(-c_{2}^{\prime}\left(\frac{r n}{L}\right)^{d-1}\right)+c_{1} \mathrm{e}^{-c_{2} n^{1-3 / 2 d}} .
\end{aligned}
$$

The result follows.

\section{Lower large deviations and shape Theorem}

6.1. Closeness to the set of Wulff shapes. The aim of this section is to prove Theorem 1.7.

Proof of Theorem 1.\%: Let $\varepsilon>0$. Let $\xi>0$ that we will choose later depending on $\varepsilon$. We define $\lambda$ such that

$$
1-\lambda=\frac{1}{1+\xi} \text {. }
$$

We denote by $W_{\xi}$ :

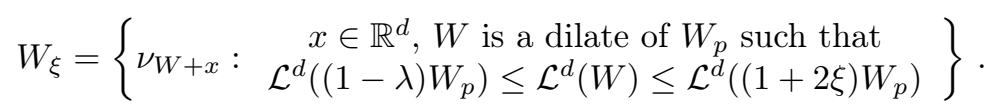

Let $u:] 0,+\infty[\rightarrow] 0,+\infty\left[\right.$ be a non-decreasing function such that $\lim _{t \rightarrow 0} u(t)=0$. Let $n \geq 1$. Let $\mathfrak{F}_{n}$ be a finite set of uniformly continuous function that satisfies for all $f \in \mathfrak{F}_{n}$,

$$
\|f\|_{\infty} \leq 1 \quad \text { and } \quad \forall x, y \in \mathbb{R}^{d},|f(x)-f(y)| \leq u\left(\|x-y\|_{2}\right) .
$$

We define the weak neighborhood $\mathcal{V}\left(\mathcal{W}_{\xi}, \mathfrak{F}_{n}, \varepsilon\right)$ of $\mathcal{W}_{\xi}$ given $\mathfrak{F}_{n}$ and $\varepsilon$ as

$$
\mathcal{V}\left(\mathcal{W}_{\xi}, \mathfrak{F}_{n}, \varepsilon\right)=\left\{\nu \in \mathcal{M}\left(\mathbb{R}^{d}\right): \exists \mu \in \mathcal{W}_{\xi}, \sup _{f \in \mathfrak{F}_{n}}|\nu(f)-\mu(f)| \leq \varepsilon\right\} .
$$

Our goal is to show that $\mu_{n}$ is in the set $\mathcal{V}\left(\mathcal{W}_{\xi}, \mathfrak{F}_{n}, \varepsilon\right)$ with high probability.

Step (i): Let $G_{n} \in \mathcal{G}_{n}$. Thanks to Proposition 5.10, the measures $\mu_{n}$ and $\nu_{n}$ associated with $P_{n}$ and $G_{n}$ are locally close to each other. In the following, it will be more convenient to work with the continuous object $P_{n}$ instead of $G_{n}$. We can localize almost all the volume of $P_{n}$ in a random region that is a union of balls of constant radius. We follow the method in Chapter 17 in Cerf (2006). We can cover $P_{n}$ in $\mathbb{R}^{d}$, up to a small fractional volume, by a finite number of random disjoint balls of constant size. Thanks to the isoperimetric inequalities, we can then control the volume of $P_{n}$ outside of these balls. Let $\delta>0$ be a real number that we will choose later. We denote by $X$ :

$$
X=\left\{x \in \mathbb{Z}^{d}: \mathcal{L}^{d}\left(B(x, 1) \cap P_{n}\right) \geq \delta\right\} .
$$


On the event $\left\{\left|\widetilde{\Gamma}_{n}\right| \leq \beta n^{d-1}\right\}$, the set $X$ is included in $B\left(0, \beta n^{d-2}\right)$ and is therefore finite. As each point in $\mathbb{R}^{d}$ belongs to at most $2^{d}$ balls among the $B(x, 1), x \in \mathbb{Z}^{d}$, then using Proposition 2.4

$$
\delta|X| \leq \sum_{x \in X} \mathcal{L}^{d}\left(B(x, 1) \cap P_{n}\right) \leq 2^{d} \mathcal{L}^{d}\left(P_{n}\right) \leq 2^{d} c_{\text {iso }} \mathcal{P}\left(P_{n}\right)^{\frac{d}{d-1}} \leq 2^{d} c_{\text {iso }} \beta^{\frac{d}{d-1}}
$$

and finally $|X| \leq M$ where $M=2^{d} c_{i s o} \beta^{\frac{d}{d-1}} / \delta$. We now would like to control the volume of $P_{n}$ outside the balls $B(x, 1)$ in $X$, i.e., to bound the measure of $P_{n} \backslash \bigcup_{x \in X} B(x, 1)$. For $x \in \mathbb{Z}^{d} \backslash X$, by the isoperimetric inequality in Proposition 2.4, we obtain as in section 17 in Cerf (2006)

$$
\begin{aligned}
\mathcal{L}^{d}\left(P_{n} \backslash \bigcup_{x \in X} B(x, 1)\right) & \leq \sum_{x \in \mathbb{Z}^{d} \backslash X} \mathcal{L}^{d}\left(P_{n} \cap B(x, 1)\right) \\
& \leq \delta^{1 / d} b_{i s o}^{\frac{d}{d-1}} \sum_{x \in \mathbb{Z}^{d} \backslash X} \mathcal{P}\left(P_{n}, \stackrel{\circ}{B}(x, 1)\right) \\
& =\delta^{1 / d} b_{\text {iso }}^{\frac{d}{d-1}} \sum_{x \in \mathbb{Z}^{d} \backslash X} \mathcal{H}^{d-1}\left(\partial^{*} P_{n} \cap \stackrel{\circ}{B}(x, 1)\right) \\
& \leq 2^{d} \delta^{1 / d} b_{i s o}^{\frac{d}{d-1}} \mathcal{H}^{d-1}\left(\partial^{*}\left(P_{n}\right)\right)=2^{d} \delta^{1 / d} b_{\text {iso }}^{\frac{d}{d-1}} \mathcal{P}\left(P_{n}\right) \\
& \leq 2^{d} \delta^{1 / d} b_{\text {iso }}^{\frac{d}{d-1}} \beta .
\end{aligned}
$$

We note $\eta=2^{d} \delta^{1 / d} b_{\text {iso }}^{\frac{d}{d-1}} \beta$. Therefore, if $\mathcal{P}\left(P_{n}\right) \leq \beta$, then $X \subset B\left(0, \beta n^{d-2}\right)$, $|X| \leq M$ and $\mathcal{L}^{d}\left(P_{n} \backslash \cup_{x \in X} B(x, 1)\right) \leq \eta$. We next would like to perform a kind of

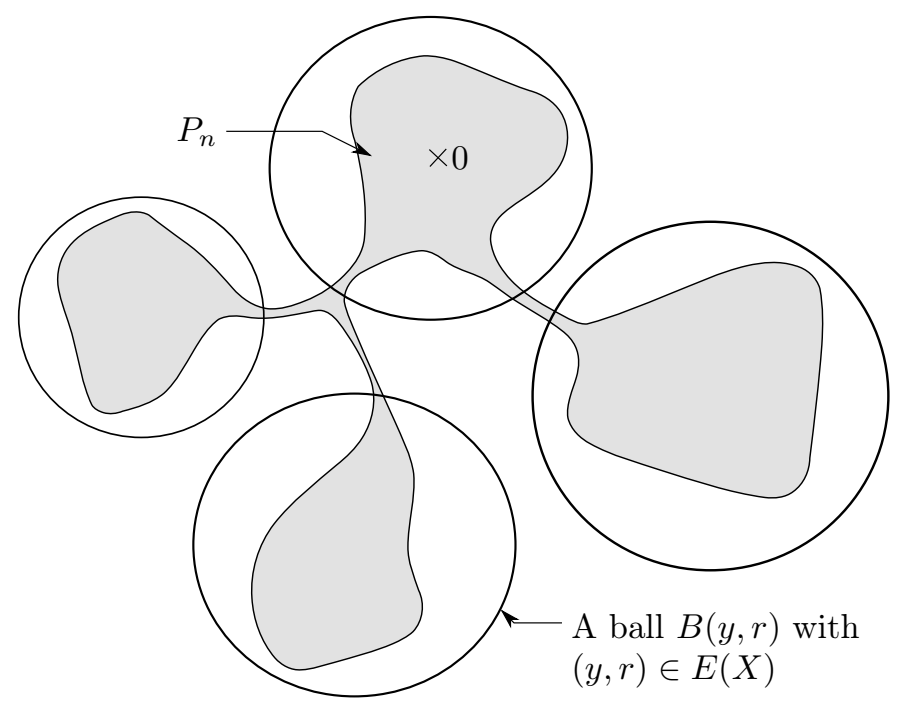

Figure 6.6. Covering almost all the volume of $P_{n}$ by balls of constant radius

surgery between the balls. To do so, we first shall build from the balls $(B(x, 1))_{x \in X}$ 
a family of balls that covers $\cup_{x \in X} B(x, 1)$ and such that the balls are far apart (see Figure 6.6). This is the purpose of Lemma 17.1. in Cerf (2006). We obtain a subset

$$
E(X)=\left\{\left(y_{1}, r_{1}\right), \ldots,\left(y_{m}, r_{m}\right)\right\} \subset X \times\left\{1, \ldots, 3^{|X|}\right\}
$$

such that $|E(X)| \leq|X|$ and

- $\forall(a, r) \in E(X), B(a, r) \cap X \neq \emptyset$

- $\cup_{x \in X} B(x, 1) \subset \cup_{(a, r) \in E(X)} B(a, r)$

- $\forall(a, r),(b, s) \in E(X),(a, r) \neq(b, s) \Rightarrow B(a, r+1) \cap B(b, s+1)=\emptyset$

We set

$$
\varphi_{W_{p}}=\frac{\mathcal{I}_{p}\left(W_{p}\right)}{\theta_{p} \mathcal{L}^{d}\left(W_{p}\right)} .
$$

Let $\delta^{\prime}>0$ be a real number that we will choose later. By applying Corollary 5.8 and Theorem 1.4, we obtain by conditioning on $E(X)$,

$$
\begin{aligned}
\mathbb{P}\left(\exists G_{n} \in \mathcal{G}_{n}, \mu_{n} \notin \mathcal{V}\left(\mathcal{W}_{\xi}, \mathfrak{F}_{n}, \varepsilon\right) \mid 0 \in \mathcal{C}_{\infty}\right) \\
\leq \\
\quad \underline{\mathbb{P}}\left(\max _{G_{n} \in \mathcal{G}_{n}} \mathcal{P}\left(n P_{n}\right) \geq \beta n^{d-1}\right)+\mathbb{P}\left(n \varphi_{n}>\left(1+\delta^{\prime}\right) \varphi_{W_{p}}\right) \\
\left.\quad+\underline{\mathbb{P}}\left(\exists G_{n} \in \mathcal{G}_{n}, \mu_{n} \notin \mathcal{V}\left(\mathcal{W}_{\xi}, \mathfrak{F}_{n}, \varepsilon\right), \mathcal{P}\left(P_{n}\right) \leq \beta, n \varphi_{n} \leq\left(1+\delta^{\prime}\right) \varphi_{W_{p}}\right]\right) \\
\leq b_{1} \exp \left(-b_{2} n^{1-3 / 2 d}\right)+b_{1}^{\prime} \exp \left(-b_{2}^{\prime} n\right) \\
\quad+\sum_{1 \leq m \leq M} \sum_{y_{1}, \ldots, y_{m}} \sum_{r_{1}, \ldots, r_{m}} \mathbb{P}\left(\begin{array}{c}
\exists G_{n} \in \mathcal{G}_{n}, \mu_{n} \notin \mathcal{V}\left(\mathcal{W}_{\xi}, \mathfrak{F}_{n}, \varepsilon\right), \\
E(X)=\left\{\left(y_{1}, r_{1}\right), \ldots,\left(y_{m}, r_{m}\right)\right\} \\
\mathcal{P}\left(P_{n}\right) \leq \beta, n \varphi_{n} \leq\left(1+\delta^{\prime}\right) \varphi_{W_{p}}
\end{array}\right)
\end{aligned}
$$

where the second summation is over $y_{1}, \ldots, y_{m}$ in $\mathbb{Z}^{d} \cap B\left(0, \beta n^{d-2}\right)$ and the third summation is over $r_{1}, \ldots, r_{m}$ in $\left\{1, \ldots, 3^{M}\right\}$. The number of ways to choose $m$ and $r_{1}, \ldots, r_{m}$ is bounded from above by a constant depending only on $M$, while the number of ways of choosing the centers $y_{1}, \ldots, y_{m}$ is polynomial in $n$. We next control the probability inside the sums. We will only focus on what happens inside the balls.

Step (ii): Let $\left\{\left(y_{1}, r_{1}\right), \ldots,\left(y_{m}, r_{m}\right)\right\}$ be a value for the random set $E(X)$ which occurs with positive probability. We define $\Omega=\Omega(E(X))$ as

$$
\Omega=\bigcup_{i=1}^{m} \stackrel{\circ}{B}\left(y_{i}, r_{i}+1\right),
$$

and the restriction $\bar{P}_{n}$ of $P_{n}$ to the balls determined by $E(X)$ :

$$
\bar{P}_{n}=P_{n} \cap\left(\bigcup_{i=1}^{m} \stackrel{\circ}{B}\left(y_{i}, r_{i}+1\right)\right) .
$$

Thus, using inequality (6.1), we have

$$
\mathcal{L}^{d}\left(P_{n} \backslash \bar{P}_{n}\right) \leq \eta
$$

We show now that $\nu_{\bar{P}_{n}}(f)$ is close to $\mu_{n}(f)$ with high probability on the event

$$
\left\{E(X)=\left\{\left(y_{1}, r_{1}\right), \ldots,\left(y_{m}, r_{m}\right)\right\}\right\} .
$$

It is easy to check that $\mathfrak{F}_{n} \cup\{1\}$ associated with the function $u$ satisfies the conditions required in Proposition 5.10. So that applying Proposition 5.10 for every $r \in$ 
$\left\{1, \ldots, 3^{M}\right\}$, there exist positive constants $c_{1}, c_{2}$ depending on $M, u$, and $\delta$ such that for all $x \in \mathbb{Z}^{d}$

$$
\begin{aligned}
\max _{r \in\left\{1, \ldots, 3^{M}\right\}} \mathbb{P}\left(\max _{G_{n} \in \mathcal{G}_{n}} \sup _{f \in \widetilde{F}_{n} \cup\{1\}}\left|\nu_{n}\left(f \mathbb{1}_{B(x, r)}\right)-\mu_{n}\left(f \mathbb{1}_{B(x, r)}\right)\right|\right. & \left.>\frac{\eta}{M}\right) \\
& \leq c_{1} \mathrm{e}^{-c_{2} n^{1-3 / 2 d}} .
\end{aligned}
$$

Thus, using inequality (6.3), we obtain

$$
\begin{aligned}
& \mathbb{P}\left(\max _{G_{n} \in \mathcal{G}_{n}} \sup _{f \in \mathfrak{F}_{n} \cup\{1\}}\left|\nu_{\bar{P}_{n}}(f)-\mu_{n}(f)\right|>2 \eta, E(X)=\left\{\left(y_{1}, r_{1}\right), \ldots,\left(y_{m}, r_{m}\right)\right\}\right) \\
& \quad \leq \sum_{i=1}^{m} \mathbb{P}\left(\max _{G_{n} \in \mathcal{G}_{n}} \sup _{f \in \mathfrak{F}_{n} \cup\{1\}}\left|\nu_{\bar{P}_{n}}\left(f \mathbb{1}_{B\left(y_{i}, r_{i}\right)}\right)-\mu_{n}\left(f \mathbb{1}_{B\left(y_{i}, r_{i}\right)}\right)\right|>\eta / M\right) \\
& \quad \leq M \max _{r \in\left\{1, \ldots, 3^{M}\right\}} \mathbb{P}\left(\max _{G_{n} \in \mathcal{G}_{n}} \sup _{f \in \mathfrak{F}_{n} \cup\{1\}}\left|\nu_{n}\left(f \mathbb{1}_{B\left(y_{1}, r\right)}\right)-\mu_{n}\left(f \mathbb{1}_{B\left(y_{1}, r\right)}\right)\right|>\eta / M\right) \\
& \quad \leq M c_{1} \mathrm{e}^{-c_{2} n^{1-3 / 2 d}} .
\end{aligned}
$$

In particular, on the event $\left\{E(X)=\left\{\left(y_{1}, r_{1}\right), \ldots,\left(y_{m}, r_{m}\right)\right\}\right\}$, with probability at least $1-M c_{1} \exp \left(-c_{2} n^{1-3 / 2 d}\right)$, we have

$$
\left|\theta_{p} \mathcal{L}^{d}\left(\bar{P}_{n}\right)-\frac{\left|G_{n}\right|}{n^{d}}\right| \leq 2 \eta
$$

Moreover, by Lemma 5.1, there exist positive constants $\eta_{1}, D_{1}$ and $D_{2}$ such that

$$
\mathbb{P}\left(\min _{G_{n} \in \mathcal{G}_{n}}\left|G_{n}\right| \leq \eta_{1} n^{d}\right) \leq D_{1} \exp \left(-D_{2} n^{(d-1) / 2 d)}\right) .
$$

We recall that $\eta$ is a function of $\delta$. We will choose $\delta$ small enough such that

$$
\eta \leq \min \left(\frac{\eta_{1}}{4}, \frac{\xi^{d}}{2}, \frac{\varepsilon}{8}, \frac{\eta_{1}}{3 \theta_{p}}\right) .
$$

Other conditions will be imposed later on $\delta$.

On the event $\left\{\min _{G_{n} \in \mathcal{G}_{n}}\left|G_{n}\right|>\eta_{1} n^{d}\right\}$, using inequalities (6.5) and (6.6), we obtain

$$
\mathcal{L}^{d}\left(\bar{P}_{n}\right) \geq \frac{1}{\theta_{p}}\left(\frac{\left|G_{n}\right|}{n^{d}}-2 \eta\right) \geq \frac{1}{\theta_{p}}\left(\eta_{1}-2 \eta\right) \geq \frac{\eta_{1}}{2 \theta_{p}}
$$

and as $\mathcal{L}^{d}\left(W_{p}\right)=1 / \theta_{p}$, using inequality (6.5), we have

$$
\mathcal{L}^{d}\left(\bar{P}_{n}\right) \leq \frac{1}{\theta_{p}}\left(\frac{\left|G_{n}\right|}{n^{d}}+2 \eta\right) \leq \frac{1}{\theta_{p}}\left(1+\xi^{d}\right)=\mathcal{L}^{d}\left(W_{p}\right)\left(1+\xi^{d}\right) \leq \mathcal{L}^{d}\left((1+\xi) W_{p}\right) .
$$

For $\nu \in \mathcal{W}_{\xi}$, we have

$$
\sup _{f \in \widetilde{F}_{n}}\left|\nu_{\bar{P}_{n}}(f)-\nu(f)\right| \geq \sup _{f \in \widetilde{F}_{n}}\left|\mu_{n}(f)-\nu(f)\right|-\sup _{f \in \widetilde{F}_{n}}\left|\mu_{n}(f)-\nu_{\bar{P}_{n}}(f)\right|,
$$

so that, together with inequalities (6.4) and (6.6), with high probability,

$$
\mu_{n} \notin \mathcal{V}\left(\mathcal{W}_{\xi}, \mathfrak{F}_{n}, \varepsilon\right) \Longrightarrow \nu_{\bar{P}_{n}} \notin \mathcal{V}\left(\mathcal{W}_{\xi}, \mathfrak{F}_{n}, 3 \varepsilon / 4\right)
$$


Thus, combining with inequalities (6.7) and (6.8), we have

$$
\begin{aligned}
& \underline{\mathbb{P}}\left(\begin{array}{c}
\exists G_{n} \in \mathcal{G}_{n}, \mu_{n} \notin \mathcal{V}\left(\mathcal{W}_{\xi}, \mathfrak{F}_{n}, \varepsilon\right), \mathcal{P}\left(P_{n}\right) \leq \beta, \\
E(X)=\left\{\left(y_{1}, r_{1}\right), \ldots,\left(y_{m}, r_{m}\right)\right\}, n \varphi_{n} \leq\left(1+\delta^{\prime}\right) \varphi_{W_{p}}
\end{array}\right) \\
& \quad \leq \underline{\mathbb{P}}\left(\begin{array}{c}
\exists G_{n} \in \mathcal{G}_{n}, \nu \bar{P}_{n} \notin \mathcal{V}\left(\mathcal{W}_{\xi}, \mathfrak{F}_{n}, 3 \varepsilon / 4\right), n \varphi_{n} \leq\left(1+\delta^{\prime}\right) \varphi_{W_{p}}, \\
\frac{\eta_{1}}{2 \theta_{p}} \leq \mathcal{L}^{d}\left(\bar{P}_{n}\right) \leq \mathcal{L}^{d}\left((1+\xi) W_{p}\right), E(X)=\left\{\left(y_{1}, r_{1}\right), \ldots,\left(y_{m}, r_{m}\right)\right\}
\end{array}\right) \\
& +M c_{1} \mathrm{e}^{-c_{2} n^{1-3 / 2 d}}+D_{1} \exp \left(-D_{2} n^{(d-1) / 2 d}\right) .
\end{aligned}
$$

We do not cover $\bar{P}_{n}$ directly but we cover separately each $\bar{P}_{n} \cap B\left(y_{k}, r_{k}+1\right)$ for $k \in\{1, \ldots, m\}$. For any $r \in\left\{1, \ldots, 3^{M}\right\}$, we define the space

$$
\mathcal{C}_{\beta}^{(r)}=\{F \subset \stackrel{\circ}{B}(0, r+1), \mathcal{P}(F, \stackrel{\circ}{B}(0, r+1)) \leq \beta\}
$$

endowed with the topology $L^{1}$ associated to the distance $d\left(F, F^{\prime}\right)=\mathcal{L}^{d}\left(F \Delta F^{\prime}\right)$, where $\Delta$ is the symmetric difference between sets. For this topology, the space $\mathcal{C}_{\beta}^{(r)}$ is compact. Suppose that we associate to each $F \in \mathcal{C}_{\beta}^{(r)}$ a positive number $\varepsilon_{F} \leq \min \left(\eta, \mathcal{L}^{d}\left(\xi W_{p}\right)\right) / M$. The collection of open sets

$$
\left\{H \text { Borel subset of } \stackrel{\circ}{B}(0, r+1): \mathcal{L}^{d}(H \Delta F)<\varepsilon_{F}\right\}, F \in \mathcal{C}_{\beta}^{(r)},
$$

is then an open covering of $\mathcal{C}_{\beta}^{(r)}$. By compactness, we can extract a finite covering $\left(F_{i}^{(r)}, \varepsilon_{F_{i}^{(r)}}\right)_{1 \leq i \leq N^{(r)}}$ of $\mathcal{C}_{\beta}^{(r)}$. By union bound, we obtain

$$
\begin{array}{r}
\exists G_{n} \in \mathcal{G}_{n}, \nu_{\bar{P}_{n}} \notin \mathcal{V}\left(\mathcal{W}_{\xi}, \mathfrak{F}_{n}, 3 \varepsilon / 4\right), n \varphi_{n} \leq\left(1+\delta^{\prime}\right) \varphi_{W_{p}}, \\
\underline{\mathbb{P}}\left(\begin{array}{c}
\eta_{1} \\
\frac{\eta_{p}}{2 \theta_{p}} \leq \mathcal{L}^{d}\left(\bar{P}_{n}\right) \leq \mathcal{L}^{d}\left((1+\xi) W_{p}\right), E(X)=\left\{\left(y_{1}, r_{1}\right), \ldots,\left(y_{m}, r_{m}\right)\right\}
\end{array}\right) \\
\leq \sum_{i_{1}=1}^{N^{\left(r_{1}\right)}} \cdots \sum_{i_{m}=1}^{N^{\left(r_{m}\right)}} \mathbb{P}\left(\mathcal{F}_{i_{1}, \ldots, i_{m}}\right)
\end{array}
$$

where

$$
\mathcal{F}_{i_{1}, \ldots, i_{m}}=\left\{\begin{array}{c}
\exists G_{n} \in \mathcal{G}_{n}: \forall 1 \leq k \leq m, \\
\mathcal{L}^{d}\left(\left(F_{i_{k}}^{\left(r_{k}\right)}+y_{k}\right) \Delta\left(\bar{P}_{n} \cap B\left(y_{k}, r_{k}+1\right)\right) \leq \varepsilon_{F_{i_{k}}}^{\left(r_{k}\right)},\right. \\
\nu_{\bar{P}_{n}} \notin \mathcal{V}\left(\mathcal{W}_{\xi}, \mathfrak{F}_{n}, 3 \varepsilon / 4\right), n \varphi_{n} \leq\left(1+\delta^{\prime}\right) \varphi_{W_{p}}, \\
\frac{\eta_{1}}{2 \theta_{p}} \leq \mathcal{L}^{d}\left(\bar{P}_{n}\right) \leq \mathcal{L}^{d}\left((1+\xi) W_{p}\right), \\
E(X)=\left\{\left(y_{1}, r_{1}\right), \ldots,\left(y_{m}, r_{m}\right)\right\}
\end{array}\right\} .
$$

So we need to study the quantity $\underline{\mathbb{P}}(\mathcal{F})$ for a generic $m$-uplet $\left(F_{1}, \ldots, F_{m}\right) \in \mathcal{C}_{\beta}^{\left(r_{1}\right)} \times$ $\cdots \times \mathcal{C}_{\beta}^{\left(r_{m}\right)}$ and their associated $\varepsilon_{F_{1}}, \ldots, \varepsilon_{F_{m}}$. By definition of the Cheeger constant $\varphi_{n}$, we obtain

$$
\underline{\mathbb{P}}(\mathcal{F})=\mathbb{P}\left(\begin{array}{c}
\forall 1 \leq i \leq m, \\
\mathcal{L}^{d}\left(\left(F_{i}+y_{i}\right) \Delta\left(\bar{P}_{n} \cap B\left(y_{i}, r_{i}+1\right)\right)\right) \leq \varepsilon_{F_{i}}, \\
\nu \bar{P}_{n} \notin \mathcal{V}\left(\mathcal{W}_{\xi}, \mathfrak{F}_{n}, 3 \varepsilon / 4\right), \\
\exists G_{n} \in \mathcal{G}_{n}: \quad \\
\left|\partial^{o} G_{n}\right| \leq\left(1+\delta^{\prime}\right) n^{-1}\left|G_{n}\right| \varphi_{W_{p}}, \\
\frac{\eta_{1}}{2 \theta_{p}} \leq \mathcal{L}^{d}\left(\bar{P}_{n}\right) \leq \mathcal{L}^{d}\left((1+\xi) W_{p}\right), \\
E(X)=\left\{\left(y_{1}, r_{1}\right), \ldots,\left(y_{m}, r_{m}\right)\right\}
\end{array}\right) .
$$


To lighten the notations, we set

$$
F=\bigcup_{i=1}^{m}\left(F_{i}+y_{i}\right)
$$

We have

$$
\begin{aligned}
\mathcal{L}^{d}\left(F \Delta \bar{P}_{n}\right) & =\sum_{i=1}^{m} \mathcal{L}^{d}\left(\left(\bar{P}_{n} \cap B\left(y_{i}, r_{i}+1\right)\right) \Delta\left(F_{i}+y_{i}\right)\right) \\
& \leq \sum_{i=1}^{m} \varepsilon_{F_{i}} \leq \min \left(\eta, \mathcal{L}^{d}\left(\xi W_{p}\right)\right)
\end{aligned}
$$

Whereas the surface tension of $F$ in the interior of these balls corresponds to the surface tension of our minimizer $G_{n}$, the surface tension of $F$ along the boundary of the balls $B\left(y_{j}, r_{j}+1\right)$ does not correspond to the surface tension of $G_{n}$ because we have artificially created it. Roughly speaking, $F$ is the continuous object corresponding to the graph $G_{n}$ intersected with the $n B\left(y_{j}, r_{j}+1\right)$. This new graph has extra surface tension compared to $G_{n}$ due to the fact that we have built it by cutting $G_{n}$ along the boundary of these balls. However, our hope is to cut along the boundary of these balls in such a way that the surface tension we create is negligible. We do not work on $G_{n}$ but on the continuous object $F$, but we have to keep in mind that these two objects are close. The idea is to cut $F$ in the regions $B\left(y_{i}, r_{i}+1\right) \backslash B\left(y_{i}, r_{i}\right), i \in\{1, \ldots, m\}$. These regions contain a negligible volume of $G_{n}$ and so of $F$, we want to cut $F$ in these regions along a surface of negligible perimeter and so of negligible surface tension. By Lemma 14.4 in Cerf (2006), for $i \in\{1, \ldots, m\}$, for $\mathcal{H}^{1}$ almost all $t$ in $] 0,1[$,

$$
\mathcal{I}\left(F \cap B\left(y_{i}, r_{i}+t\right)\right) \leq \mathcal{I}\left(F \cap \stackrel{\circ}{B}\left(y_{i}, r_{i}+t\right)\right)+\beta_{\max } \mathcal{H}^{d-1}\left(F \cap \partial B\left(y_{i}, r_{i}+t\right)\right) .
$$

Let $T$ be a subset of ]0,1[ where all the above inequalities hold simultaneously. We recall that for any $i \in\{1, \ldots, m\}, \varepsilon_{F_{i}} \leq \eta / M$. We have $\mathcal{H}^{1}(T)=1$ and when we integrate in polar coordinates, using inequality (6.11),

$$
\begin{aligned}
\int_{T} \sum_{i=1}^{m} \mathcal{H}^{d-1}\left(F \cap \partial B\left(y_{i}, r_{i}+t\right)\right) d t & =\sum_{i=1}^{m} \mathcal{L}^{d}\left(F \cap B\left(y_{i}, r_{i}+1\right) \backslash B\left(y_{i}, r_{i}\right)\right) \\
& \leq \sum_{i=1}^{m} \mathcal{L}^{d}\left(\left(F_{i}+y_{i}\right) \backslash B\left(y_{i}, r_{i}\right)\right) \\
& \leq \mathcal{L}^{d}\left(\bar{P}_{n} \backslash \bigcup_{i=1}^{m} B\left(y_{i}, r_{i}\right)\right)+\mathcal{L}^{d}\left(\bar{P}_{n} \Delta F\right) \\
& \leq 2 \eta
\end{aligned}
$$

Thus, there exists $t \in T$ such that

$$
\sum_{i=1}^{m} \mathcal{H}^{d-1}\left(F \cap \partial B\left(y_{i}, r_{i}+t\right)\right) \leq 3 \eta .
$$

We next set

$$
\bar{F}=F \cap\left(\bigcup_{i=1}^{m} B\left(y_{i}, r_{i}+t\right)\right) .
$$


Using inequality (6.13), we get

$$
\begin{aligned}
\mathcal{P}(\bar{F}) & \leq \mathcal{P}\left(\bar{F}, \bigcup_{i=1}^{m} \stackrel{\circ}{B}\left(y_{i}, r_{i}+t\right)\right)+\sum_{i=1}^{m} \mathcal{H}^{d-1}\left(F \cap \partial B\left(y_{i}, r_{i}+t\right)\right) \\
& \leq \mathcal{P}\left(\bar{F}, \bigcup_{i=1}^{m} \stackrel{\circ}{B}\left(y_{i}, r_{i}+t\right)\right)+3 \eta
\end{aligned}
$$

and using Proposition 2.3,

$$
\mathcal{I}_{p}(\bar{F}) \leq \mathcal{I}_{p}\left(\bar{F}, \bigcup_{i=1}^{m} \stackrel{\circ}{B}\left(y_{i}, r_{i}+t\right)\right)+3 \beta_{\max } \eta .
$$

On the event $\mathcal{F}$, using inequality (6.11), we obtain

$$
\begin{aligned}
\mathcal{L}^{d}(F) & \leq \mathcal{L}^{d}(\bar{F})+\mathcal{L}^{d}\left(F \backslash \bigcup_{i=1}^{m} B\left(y_{i}, r_{i}\right)\right) \\
& \leq \mathcal{L}^{d}(\bar{F})+\mathcal{L}^{d}\left(F \Delta \bar{P}_{n}\right)+\mathcal{L}^{d}\left(\bar{P}_{n} \backslash \bigcup_{i=1}^{m} B\left(y_{i}, r_{i}\right)\right) \leq \mathcal{L}^{d}(\bar{F})+2 \eta .
\end{aligned}
$$

Finally, using inequalities (6.14) and (6.16), we obtain

$$
\begin{aligned}
\mathcal{I}_{p}\left(\bar{F}, \bigcup_{i=1}^{m} \stackrel{\circ}{B}\left(y_{i}, r_{i}+t\right)\right) & \geq \beta_{\text {min }} \mathcal{P}\left(\bar{F}, \bigcup_{i=1}^{m} \stackrel{\circ}{B}\left(y_{i}, r_{i}+t\right)\right) \\
& \geq \beta_{\min }(\mathcal{P}(\bar{F})-3 \eta) .
\end{aligned}
$$

and using again inequality (6.11),

$$
\mathcal{L}^{d}(F) \geq \mathcal{L}^{d}\left(\bar{P}_{n}\right)-\mathcal{L}^{d}\left(\bar{P}_{n} \Delta F\right) \geq \frac{\eta_{1}}{2 \theta_{p}}-\eta .
$$

Using the isoperimetric inequalities of Proposition 2.4 and inequalities (6.16) and (6.18), we get

$$
\mathcal{P}(\bar{F}) \geq\left(\frac{\mathcal{L}^{d}(\bar{F})}{c_{\text {iso }}}\right)^{1-1 / d} \geq\left(\frac{\mathcal{L}^{d}(F)-2 \eta}{c_{\text {iso }}}\right)^{1-1 / d} \geq\left(\frac{\eta_{1}-6 \eta \theta_{p}}{2 \theta_{p} c_{i s o}}\right)^{1-1 / d} .
$$

Next, we choose $\delta$ small enough to obtain a $\eta$ that satisfies the following inequalities:

$$
3 \beta_{\max } \eta \leq \frac{\lambda \beta_{\min }}{2}\left(\left(\frac{\eta_{1}-6 \eta \theta_{p}}{2 \theta_{p} c_{i s o}}\right)^{1-1 / d}-3 \eta\right),
$$

and also

$$
\eta_{1} \geq 6 \eta \theta_{p} .
$$

With this choice of $\delta$, we obtain with high probability, using inequalities (6.15), (6.17) and (6.19),

$$
\mathcal{I}_{p}(\bar{F}) \leq(1+\lambda / 2) \mathcal{I}_{p}\left(\bar{F}, \bigcup_{i=1}^{m} \stackrel{\circ}{B}\left(y_{i}, r_{i}+t\right)\right) \leq(1+\lambda / 2) \mathcal{I}_{p}(F, \Omega) .
$$

Let $G_{n} \in \mathcal{G}_{n}$, on the event $\mathcal{F}$, we have

$$
\left|\theta_{p} \mathcal{L}^{d}\left(\bar{P}_{n}\right)-\frac{\left|G_{n}\right|}{n^{d}}\right| \leq 2 \eta
$$


So that, together with inequality (6.16),

$$
\begin{aligned}
\left|G_{n}\right| & \leq n^{d}\left(\theta_{p} \mathcal{L}^{d}(F)+\theta_{p} \mathcal{L}^{d}\left(\bar{P}_{n} \Delta F\right)+2 \eta\right) \\
& \leq n^{d}\left(\theta_{p} \mathcal{L}^{d}(\bar{F})+\varepsilon_{F}+4 \eta\right) \\
& \leq n^{d} \theta_{p} \mathcal{L}^{d}(\bar{F})\left(1+\frac{5 \eta}{\theta_{p} \mathcal{L}^{d}(\bar{F})}\right) .
\end{aligned}
$$

Let us now choose $\delta$ small enough so that

$$
\frac{5 \eta}{\eta_{1} / 2-3 \eta \theta_{p}} \leq \delta^{\prime}
$$

Using inequalities (6.16) and (6.18), we obtain

$$
\begin{aligned}
\left|G_{n}\right| & \leq n^{d} \theta_{p} \mathcal{L}^{d}(\bar{F})\left(1+\frac{5 \eta}{\eta_{1} / 2-3 \eta \theta_{p}}\right) \\
& \leq n^{d} \theta_{p} \mathcal{L}^{d}(\bar{F})\left(1+\delta^{\prime}\right) .
\end{aligned}
$$

Finally, let $r$ be such that $\mathcal{L}^{d}(\bar{F})=\mathcal{L}^{d}\left(r W_{p}\right)$, we get

$$
\begin{aligned}
\left(1+\delta^{\prime}\right) n^{-1}\left|G_{n}\right| \varphi_{W_{p}} & \leq\left(1+\delta^{\prime}\right)^{2} n^{d-1} \frac{\varphi_{W_{p}}}{\varphi_{\bar{F}}} \mathcal{I}_{p}(\bar{F}) \\
& \leq\left(1+\delta^{\prime}\right)^{2} \frac{\mathcal{I}_{p}\left(r W_{p}\right)}{\mathcal{I}_{p}(\bar{F})} r n^{d-1} \mathcal{I}_{p}(\bar{F})
\end{aligned}
$$

We now choose $\delta^{\prime}$ small enough such that

$$
\left(1+\delta^{\prime}\right)^{2}(1-\lambda) \leq 1-\frac{\lambda}{2} .
$$

Using inequality (6.11), we obtain

$$
\mathcal{L}^{d}(\bar{F}) \leq \mathcal{L}^{d}\left(\bar{P}_{n}\right)+\mathcal{L}^{d}\left(\overline{P_{n}} \Delta F\right) \leq \mathcal{L}^{d}\left((1+\xi) W_{p}\right)+\mathcal{L}^{d}\left(\xi W_{p}\right) \leq \mathcal{L}^{d}\left((1+2 \xi) W_{p}\right)
$$

and so $r \leq 1+2 \xi$. We distinguish now two cases:

- If $r \leq 1-\lambda$, using inequality (6.23)

$$
\left(1+\delta^{\prime}\right)^{2} \frac{\mathcal{I}_{p}\left(r W_{p}\right)}{\mathcal{I}_{p}(\bar{F})} r n^{d-1} \mathcal{I}_{p}(\bar{F}) \leq(1-\lambda / 2) n^{d-1} \mathcal{I}_{p}(\bar{F})
$$

where we used the fact that the Wulff crystal is a minimizer for $\mathcal{I}_{p}$, i.e., that $\mathcal{I}_{p}\left(r W_{p}\right) \leq \mathcal{I}_{p}(\bar{F})$.

- Let us assume that $r \in(1-\lambda, 1+2 \xi]$. We recall that on the event $\mathcal{F}$, for all $\nu \in \mathcal{W}_{\xi}$,

$$
\sup _{f \in \widetilde{F}_{n}}\left|\nu_{\bar{P}_{n}}(f)-\nu(f)\right| \geq 3 \varepsilon / 4 .
$$

Thus, for all $x \in \mathbb{R}^{d}$, for $f \in \mathfrak{F}_{n}$ we have

$$
\begin{aligned}
\left|\nu_{\bar{P}_{n}}(f)-\nu_{r W_{p}+x}(f)\right| & \leq\left|\int_{\bar{P}_{n} \backslash\left(r W_{p}+x\right)} f(x) d \mathcal{L}^{d}(x)-\int_{\left(r W_{p}+x\right) \backslash \bar{P}_{n}} f(x) d \mathcal{L}^{d}(x)\right| \\
& \leq \int_{\bar{P}_{n} \backslash\left(r W_{p}+x\right)}|f(x)| d \mathcal{L}^{d}(x)+\int_{\left(r W_{p}+x\right) \backslash \bar{P}_{n}}|f(x)| d \mathcal{L}^{d}(x) \\
& \leq \int_{\bar{P}_{n} \backslash\left(r W_{p}+x\right)} 1 d \mathcal{L}^{d}(x)+\int_{\left(r W_{p}+x\right) \backslash \bar{P}_{n}} 1 d \mathcal{L}^{d}(x) \\
& \leq \mathcal{L}^{d}\left(\bar{P}_{n} \Delta\left(r W_{p}+x\right)\right),
\end{aligned}
$$


and so,

$$
\mathcal{L}^{d}\left(\bar{P}_{n} \Delta\left(r W_{p}+x\right)\right) \geq \sup _{f \in \mathfrak{F}_{n}}\left|\nu_{\bar{P}_{n}}(f)-\nu_{r} W_{p}+x(f)\right| \geq 3 \varepsilon / 4
$$

and as $\eta$ satisfies inequality (6.6), we obtain

$$
\mathcal{L}^{d}\left(\bar{F} \Delta\left(r W_{p}+x\right)\right) \geq \mathcal{L}^{d}\left(\bar{P}_{n} \Delta\left(r W_{p}+x\right)\right)-\mathcal{L}^{d}\left(\bar{F} \Delta \bar{P}_{n}\right) \geq 3 \varepsilon / 4-\eta \geq \varepsilon / 2 .
$$

Moreover, as $r W_{p}$ is a minimizer for the isoperimetric problem, there exists a constant $c(\varepsilon)>0$, that is a non-decreasing function of $\varepsilon$ depending also on $p$ and $r$, that goes to 0 when $\varepsilon$ goes to 0 , such that

$$
\begin{array}{r}
\inf \left\{\mathcal{I}(E): \forall x \in \mathbb{R}^{d}, \mathcal{L}^{d}\left(E \Delta\left(x+r W_{p}\right)\right) \geq \varepsilon / 2, \mathcal{L}^{d}(E)=\mathcal{L}^{d}\left(r W_{p}\right)\right\} \\
\geq \mathcal{I}_{p}\left(r W_{p}\right)(1+c(\varepsilon)) .
\end{array}
$$

Finally,

$$
\frac{\mathcal{I}_{p}\left(r W_{p}\right)}{\mathcal{I}_{p}(\bar{F})} \leq \frac{1}{1+c(\varepsilon)}
$$

and so,

$$
\left(1+\delta^{\prime}\right)^{2} \frac{\mathcal{I}_{p}\left(r W_{p}\right)}{\mathcal{I}_{p}(\bar{F})} r n^{d-1} \mathcal{I}_{p}(\bar{F}) \leq \frac{\left(1+\delta^{\prime}\right)^{2}}{1+c(\varepsilon)}(1+2 \xi) n^{d-1} \mathcal{I}_{p}(\bar{F}) .
$$

We choose $\xi$ small enough depending on $\varepsilon$ such that

$$
\frac{1+2 \xi}{1+c(\varepsilon)} \leq 1-\lambda=\frac{1}{1+\xi} .
$$

This is equivalent to choose $\xi$ such that

$$
3 \xi+2 \xi^{2} \leq c(\varepsilon) .
$$

We obtain using inequality (6.23)

$$
\left(1+\delta^{\prime}\right)^{2} \frac{\mathcal{I}_{p}\left(r W_{p}\right)}{\mathcal{I}_{p}(\bar{F})} r n^{d-1} \mathcal{I}_{p}(\bar{F}) \leq(1-\lambda / 2) n^{d-1} \mathcal{I}_{p}(\bar{F}) .
$$

Finally, combining the two cases, with $\varepsilon$ and $\delta^{\prime}$ properly chosen and inequality (6.21), we obtain

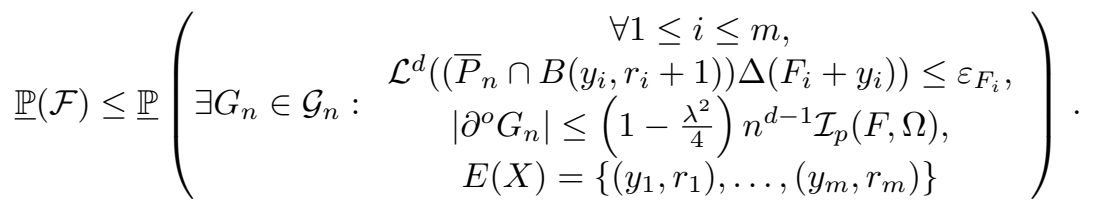

Step (iii): The remaining of the proof follows the same ideas as in Cerf and Théret (2011). We link the probability defined in the right hand side of (6.25) with the probability that the flow is abnormally small in some local region of $\partial F \cap \Omega$. We now want to cover $\partial F$ by balls of small radius such that $\partial F$ is "almost flat" in each ball, this is the purpose of the following Lemma:

Lemma 6.1 (Lemma 1 in Cerf and Théret, 2011). Let $R>0$. Let $F$ be a subset of $\stackrel{\circ}{B}(0, R)$ of finite perimeter. For every positive constants $\delta^{\prime}$ and $\eta^{\prime}$, there exists a finite family of closed disjoint balls $\left(B\left(x_{i}, \rho_{i}\right)\right)_{i \in I \cup K}$ and vectors $\left(v_{i}\right)_{i \in I \cup K}$, such that, letting $B_{i}=B\left(x_{i}, \rho_{i}\right)$ and $B_{i}^{-}=B^{-}\left(x_{i}, \rho_{i}, v_{i}\right)$, we have for all $i \in I$

$$
\left.x_{i} \in \partial^{*} F \cap \stackrel{\circ}{B}(0, R), \rho_{i} \in\right] 0,1\left[, B_{i} \subset \stackrel{\circ}{B}(0, R), \mathcal{L}^{d}\left(\left(F \cap B_{i}\right) \Delta B_{i}^{-}\right) \leq \delta^{\prime} \alpha_{d} \rho_{i}^{d},\right.
$$


and

$$
\mid \mathcal{I}_{p}(F, \stackrel{\circ}{B}(0, R))-\sum_{i \in I} \alpha_{d-1} \rho_{i}^{d-1}\left(\nu\left(n_{F}\left(x_{i}\right)\right) \mid \leq \eta^{\prime} .\right.
$$

We recall that $\alpha_{d}$ is the volume of the d-dimensional unit Euclidean ball.

We apply Lemma 6.1 to each $F_{k} \subset \stackrel{\circ}{B}\left(0, r_{k}+1\right)$, with $\delta_{2}>0$ that will be chosen later and $\eta^{\prime}=\lambda^{4} \mathcal{I}_{p}(F, \Omega) / 16 M$. We obtain for each $k$, a family

$$
\left(B_{i}^{(k)}\left(x_{i}^{(k)}, \rho_{i}^{(k)}, v_{i}^{(k)}\right)\right)_{i \in I^{(k)}}
$$

that does not depend on $y_{1}, \ldots, y_{m}$, so that

$$
\mid \mathcal{I}_{p}\left(F_{k}, \stackrel{\circ}{B}\left(0, r_{k}+1\right)\right)-\sum_{i \in I} \alpha_{d-1}\left(\rho_{i}^{(k)}\right)^{d-1}\left(\nu\left(n_{F_{k}}\left(x_{i}^{(k)}\right)\right) \mid \leq \eta^{\prime} .\right.
$$

We now choose

$$
\varepsilon_{F_{k}} \leq \min \left(\min _{i \in I^{(k)}} \alpha_{d}\left(\rho_{i}^{(k)}\right)^{d} \delta_{2}, \frac{\eta}{M}, \frac{\mathcal{L}^{d}\left(\xi W_{p}\right)}{M}\right),
$$

for a fixed $\delta_{2}$ that we will choose later. Besides, as the balls $B\left(y_{k}, r_{k}+1\right)$ are disjoint, for $k \in\{1, \ldots, m\}$, we have

$$
\mathcal{I}_{p}(F, \Omega)=\sum_{k=1}^{m} \mathcal{I}_{p}\left(F \cap B\left(y_{k}, r_{k}+1\right), \Omega\right)=\sum_{k=1}^{m} \mathcal{I}_{p}\left(F_{k}, \stackrel{\circ}{B}\left(0, r_{k}+1\right)\right) .
$$

Using inequality (6.26), we obtain

$$
\left|\mathcal{I}_{p}(F, \Omega)-\sum_{k=1}^{m} \sum_{i \in I^{(k)}} \alpha_{d-1}\left(\rho_{i}^{(k)}\right)^{d-1} \nu\left(n_{F_{k}}\left(x_{i}^{(k)}\right)\right)\right| \leq m \eta^{\prime} \leq \lambda^{4} \mathcal{I}_{p}(F, \Omega) / 16
$$

So, we get

$$
\mathcal{I}_{p}(F, \Omega) \leq \frac{1}{1-\lambda^{4} / 16}\left(\sum_{k=1}^{m} \sum_{i \in I^{(k)}} \alpha_{d-1}\left(\rho_{i}^{(k)}\right)^{d-1} \nu\left(n_{F_{k}}\left(x_{i}^{(k)}\right)\right)\right)
$$

and

$$
\left(1-\frac{\lambda^{2}}{4}\right) \mathcal{I}_{p}(F, \Omega) \leq \frac{1-\lambda^{2} / 4}{1-\lambda^{4} / 16}\left(\sum_{k=1}^{m} \sum_{i \in I^{(k)}} \alpha_{d-1}\left(\rho_{i}^{(k)}\right)^{d-1} \nu\left(n_{F_{k}}\left(x_{i}^{(k)}\right)\right)\right)
$$

Whence setting $w=\lambda^{2} /\left(4+\lambda^{2}\right)<1$,

$$
\left(1-\frac{\lambda^{2}}{4}\right) \mathcal{I}_{p}(F, \Omega) \leq(1-w)\left(\sum_{k=1}^{m} \sum_{i \in I^{(k)}} \alpha_{d-1}\left(\rho_{i}^{(k)}\right)^{d-1} \nu\left(n_{F_{k}}\left(x_{i}^{(k)}\right)\right)\right) .
$$

Since the balls $\left(B_{i}^{(k)}+y_{k}\right)_{1 \leq k \leq m, i \in I^{(k)}}$ are pairwise disjoint, we have

$$
\left|\partial^{o} G_{n}\right| \geq \sum_{k=1}^{m} \sum_{i \in I^{(k)}}\left|\left(\partial^{o} G_{n}\right) \cap\left(n\left(B_{i}^{(k)}+y_{k}\right)\right)\right|
$$


Using inequalities (6.28) and (6.29), we get



$$
\begin{aligned}
& \leq \mathbb{P}\left(\begin{array}{c}
\exists G_{n} \in \mathcal{G}_{n}, \mathcal{L}^{d}\left(\left(\bar{P}_{n} \cap B\left(y_{i}, r_{i}+1\right)\right) \Delta\left(F_{i}+y_{i}\right)\right) \leq \varepsilon_{F_{i}}, 1 \leq i \leq m, \\
\sum_{k=1}^{m} \sum_{i \in I^{(k)}}\left|\left(\partial^{o} G_{n}\right) \cap\left(n\left(B_{i}^{(k)}+y_{k}\right)\right)\right| \\
\leq(1-w) n^{d-1}\left(\sum_{k=1}^{m} \sum_{i \in I^{(k)}} \alpha_{d-1}\left(\rho_{i}^{(k)}\right)^{d-1} \nu\left(n_{F_{k}}\left(x_{i}^{(k)}\right)\right)\right)
\end{array}\right) .
\end{aligned}
$$

Let $k \in\{1, \ldots, m\}$. We aim to control $\operatorname{card}\left(\left(G_{n} \cap n\left(B_{i}^{(k)}+y_{k}\right)\right) \Delta\left(n\left(B_{i}^{(k)}+y_{k}\right)^{-} \cap\right.\right.$ $\left.\mathbb{Z}^{d}\right)$ ). To do so, it is more convenient to work with the graph $F_{n}$. In the following, we drop the superscript $(k)$ for clarity. With high probability, we have

$$
\begin{aligned}
\operatorname{card}\left(\left(G_{n} \cap\right.\right. & \left.\left.n\left(B_{i}^{(k)}+y_{k}\right)\right) \Delta\left(n\left(B_{i}^{(k)}+y_{k}\right)^{-} \cap \mathbb{Z}^{d}\right)\right) \\
& \leq \operatorname{card}\left(\left(F_{n} \cap n\left(B_{i}+y_{k}\right)\right) \Delta\left(n\left(B_{i}+y_{k}\right)^{-} \cap \mathbb{Z}^{d}\right)\right)+\operatorname{card}\left(F_{n} \backslash G_{n}\right) \\
& \leq \operatorname{card}\left(\left(F_{n} \cap n\left(B_{i}+y_{k}\right)\right) \Delta\left(n\left(B_{i}+y_{k}\right)^{-} \cap \mathbb{Z}^{d}\right)\right)+\eta_{3} n^{d-1 / 2(d-1)} .
\end{aligned}
$$

As $B_{i}+y_{k} \subset B\left(y_{k}, r_{k}+1\right)$, we have

$$
\begin{aligned}
\mathcal{L}^{d}\left(\left(n P_{n} \cap n\left(B_{i}+y_{k}\right)\right) \Delta\left(n\left(B_{i}+y_{k}\right)^{-}\right)\right) \leq & \mathcal{L}^{d}\left(\left(n F_{k} \cap n B_{i}\right) \Delta\left(n B_{i}^{-}\right)\right) \\
& +n^{d} \mathcal{L}^{d}\left(P_{n} \Delta\left(F_{k}+y_{k}\right)\right) \\
\leq & n^{d} \alpha_{d} \rho_{i}^{d} \delta_{2}+\varepsilon_{F_{k}} \leq 2 n^{d} \alpha_{d} \rho_{i}^{d} \delta_{2} .
\end{aligned}
$$

By the same arguments as in section 5.2 in Cerf and Théret (2011),

$$
\begin{aligned}
\operatorname{card}\left(\left(F_{n} \cap n(\right.\right. & \left.\left.\left.\left.B_{i}+y_{k}\right)\right) \Delta n\left(B_{i}+y_{k}\right)^{-}\right)\right) \\
& \leq \mathcal{L}^{d}\left(\left(\left(n P_{n} \cap n\left(B_{i}+y_{k}\right)\right) \Delta n\left(B_{i}+y_{k}\right)^{-}\right) \cap \mathbb{Z}^{d}+[-1 / 2,1 / 2]^{d}\right) \\
& \leq 2 n^{d} \alpha_{d} \rho_{i}^{d} \delta_{2}+n^{d-1} 4 d\left(\mathcal{H}^{d-1}\left(\partial B_{i}\right)+\mathcal{H}^{d-1}\left(\partial B_{i}^{-}\right)\right) .
\end{aligned}
$$

Finally, for $n$ large enough,

$$
\operatorname{card}\left(\left(G_{n} \cap n\left(B_{i}+y_{k}\right)\right) \Delta\left(n\left(B_{i}+y_{k}\right)^{-} \cap \mathbb{Z}^{d}\right)\right) \leq 4 n^{d} \alpha_{d} \rho_{i}^{d} \delta_{2} .
$$

Thus, using inequality (6.30), for large enough $n$,

$$
\begin{gathered}
\mathbb{P}\left(\begin{array}{c}
\exists G_{n} \in \mathcal{G}_{n}, \mathcal{L}^{d}\left(\left(\bar{P}_{n} \cap B\left(y_{i}, r_{i}+1\right)\right) \Delta\left(F_{i}+y_{i}\right)\right) \leq \varepsilon_{F_{i}}, 1 \leq i \leq m, \\
\left|\partial^{o} G_{n}\right| \leq\left(1-\lambda^{2} / 4\right) n^{d-1} \mathcal{I}_{p}(F, \Omega), E(X)=\left\{\left(y_{1}, r_{1}\right), \ldots,\left(y_{m}, r_{m}\right)\right\}
\end{array}\right) \\
\quad \exists G_{n} \in \mathcal{G}_{n}, \\
\leq \sum_{k=1}^{m} \sum_{i \in I^{(k)}} \mathbb{P}\left(\begin{array}{c}
\left|\left(G_{n} \cap n\left(B_{i}+y_{k}\right)\right) \Delta\left(n\left(B_{i}^{-}+y_{k}\right) \cap \mathbb{Z}^{d}\right)\right| \leq 4 \delta_{2} \alpha_{d} \rho_{i}^{d} n^{d}, \\
\left|\left(\partial^{o} G_{n}\right) \cap n\left(B_{i}+y_{k}\right)\right| \\
\leq(1-w) n^{d-1}\left(\alpha_{d-1} \rho_{i}^{d-1} \nu\left(n_{F_{k}}\left(x_{i}^{(k)}\right)\right)\right)
\end{array}\right) \\
\leq \frac{1}{\theta_{p}} \sum_{k=1}^{m} \sum_{i \in I^{(k)}} \mathbb{P}\left(G\left(x_{i}^{(k)}+y_{k}, \rho_{i}^{(k)}, n_{F_{k}}\left(x_{i}^{(k)}\right), w, \delta_{2}\right)\right)
\end{gathered}
$$

where $G\left(x, r, v, w, \delta_{2}\right)$ is the event that there exists a set $U \subset B \cap \mathbb{Z}^{d}$ such that:

$$
\operatorname{card}\left(U \Delta\left(n B^{-}(x, r, v) \cap \mathbb{Z}^{d}\right)\right) \leq 4 \delta_{2} \alpha_{d} r^{d} n^{d}
$$

and

$$
\left|\left(\partial^{o} G_{n}\right) \cap n B\right| \leq(1-w) \alpha_{d-1} r^{d-1}\left(\nu(v) n^{d-1} .\right.
$$


This event depends only on the edges inside $B(x, r, v)$ and is invariant under integer translation. So that,

$$
\begin{aligned}
& \mathbb{P}\left(\begin{array}{c}
\exists G_{n} \in \mathcal{G}_{n}, \mathcal{L}^{d}\left(\left(\bar{P}_{n} \cap B\left(y_{i}, r_{i}+1\right)\right) \Delta\left(F_{i}+y_{i}\right)\right) \leq \varepsilon_{F_{i}}, 1 \leq i \leq m, \\
\left|\partial^{o} G_{n}\right| \leq\left(1-\lambda^{2} / 4\right) n^{d-1} \mathcal{I}_{p}(F, \Omega), E(X)=\left\{\left(y_{1}, r_{1}\right), \ldots,\left(y_{m}, r_{m}\right)\right\}
\end{array}\right) \\
& \quad \leq \frac{1}{\theta_{p}} \sum_{k=1}^{m} \sum_{i \in I^{(k)}} \mathbb{P}\left(G\left(x_{i}^{(k)}, \rho_{i}^{(k)}, n_{F_{k}}\left(x_{i}^{(k)}\right), w, \delta_{2}\right)\right) .
\end{aligned}
$$

This event is a rare event. Indeed, if this event occurs, we can show that the capacity of the minimal cutset that separates the upper half part of $B(x, r, v)$ (upper half part according to the direction $v$ ) from the lower half part is abnormally small. To do so, we build from the set $U$ an almost flat cutset in the ball. The fact that $\operatorname{card}\left(U \Delta B^{-}(x, r, v)\right)$ is small implies that $\partial_{e} U$ is almost flat and is close to $\operatorname{disc}(x, r, v)$. However, this does not prevent the existence of long thin strands that might escape the ball and prevent $U$ from being a cutset in the ball. The idea is to cut these strands by adding edges at a fixed height. We have to choose the appropriate height to ensure that the extra edges we needed to add to cut these strands are not too many, so that we can control their capacity. The new set of edges we create by adding to $U$ these edges will be in a sense a cutset. The last thing to do is then to cover the $\operatorname{disc}(x, r, v)$ by hyperrectangles in order to use the estimate that the flow is abnormally small in a cylinder. This work was done in section 6 in Cerf and Théret (2011). It is possible to choose $\delta_{2}$ depending on $F_{1}, \ldots, F_{m}, G$ and $w$ such that for all $k \in\{1, \ldots, m\}$, there exist positive constants $C_{1, i}^{F_{k}}$ and $C_{2, i}^{F_{k}}$ depending on $G, d, F_{k}, i$ and $w$ so that for all $i \in I^{(k)}$,

$$
\mathbb{P}\left(G\left(x_{i}, \rho_{i}, n_{F_{k}}\left(x_{i}\right), w, \delta_{2}\right)\right) \leq C_{1, i}^{F_{k}} \exp \left(-C_{2, i}^{F_{k}} n^{d-1}\right) .
$$

Note that this upper bound is uniform on $y_{1}, \ldots, y_{m}$ but still depends on $r_{1}, \ldots, r_{m}$. Together with inequalities (6.25) and (6.32), we obtain

$$
\begin{aligned}
\underline{P}(\mathcal{F}) & \leq \underline{\mathbb{P}}\left(\begin{array}{c}
\exists G_{n} \in \mathcal{G}_{n}, \mathcal{L}^{d}\left(\left(\bar{P}_{n} \cap B\left(y_{i}, r_{i}+1\right)\right) \Delta\left(F_{i}+y_{i}\right)\right) \leq \varepsilon_{F_{i}}, 1 \leq i \leq m, \\
\left|\partial^{o} G_{n}\right| \leq\left(1-\lambda^{2} / 4\right) n^{d-1} \mathcal{I}_{p}(F, \Omega), \\
E(X)=\left\{\left(y_{1}, r_{1}\right), \ldots,\left(y_{m}, r_{m}\right)\right\}
\end{array}\right) \\
& \leq \frac{1}{\theta_{p}} \sum_{k=1}^{m} \sum_{i \in I^{(k)}} C_{1, i}^{F_{k}} \exp \left(-C_{2, i}^{F_{k}} n^{d-1}\right) .
\end{aligned}
$$

So there exist positive constants $C_{1}^{F_{1}}, \ldots, C_{1}^{F_{m}}$ and $C_{2}^{F_{1}}, \ldots, C_{2}^{F_{m}}$ such that

$$
\mathbb{P}(\mathcal{F}) \leq \sum_{k=1}^{m} C_{1}^{F_{k}} \exp \left(-C_{2}^{F_{k}} n^{d-1}\right)
$$

Combining inequalities (6.2), (6.9), (6.10) and (6.33), we obtain for small enough $\delta_{2}$,

$$
\begin{aligned}
\mathbb{P}( & \left.\exists G_{n} \in \mathcal{G}_{n}, \mu_{n} \notin \mathcal{V}\left(\mathcal{W}_{\xi}, \mathfrak{F}_{n}, \varepsilon\right) \mid 0 \in \mathcal{C}_{\infty}\right) \\
\leq & b_{1} \mathrm{e}^{-b_{2} n^{1-3 / 2 d}}+b_{1}^{\prime} \mathrm{e}^{-b_{2}^{\prime} n}+\sum_{m=1}^{M} \sum_{y_{1}, \ldots, y_{m}} \sum_{r_{1}, \ldots, r_{m}} \sum_{i_{1}=1}^{N^{\left(r_{1}\right)}} \cdots \sum_{i_{m}=1}^{N^{\left(r_{m}\right)}} \mathbb{P}\left[\mathcal{F}_{i_{1}, \ldots, i_{m}}\right] \\
& +M 3^{M^{2}} C_{d} n^{M(d-2)}\left(M c_{1} \mathrm{e}^{-c_{2} n^{1-3 / 2 d}}+D_{1} \mathrm{e}^{-D_{2} n^{(d-1) / 2 d}}\right) \\
& \leq b_{1} \mathrm{e}^{-b_{2} n^{1-3 / 2 d}}+b_{1}^{\prime} \mathrm{e}^{-b_{2}^{\prime} n}
\end{aligned}
$$




$$
\begin{aligned}
& +\sum_{m=1}^{M} \sum_{y_{1}, \ldots, y_{m}} \sum_{r_{1}, \ldots, r_{m}} \sum_{i_{1}=1}^{N^{\left(r_{1}\right)}} \cdots \sum_{i_{m}=1}^{N^{\left(r_{m}\right)}} \sum_{k=1}^{m} \frac{C_{1}^{F_{i_{k}}^{\left(r_{k}\right)}}}{\theta_{p}} \mathrm{e}^{-C_{2}^{F_{i_{k}}^{\left(r_{k}\right)}} n^{d-1}} \\
& \quad+M 3^{M^{2}} C_{d} n^{M(d-2)}\left(M c_{1} \mathrm{e}^{-c_{2} n^{1-3 / 2 d}}+D_{1} \mathrm{e}^{-D_{2} n^{(d-1) / 2 d}}\right) \\
& \leq b_{1} \mathrm{e}^{-b_{2} n^{1-3 / 2 d}}+b_{1}^{\prime} \mathrm{e}^{-b_{2}^{\prime} n} \\
& +\sum_{m=1}^{M} \sum_{y_{1}, \ldots, y_{m}} 3^{M^{2}} \max _{r_{1}, \ldots, r_{m}}\left\{\sum_{i_{1}=1}^{N^{\left(r_{1}\right)}} \cdots \sum_{i_{m}=1}^{N^{\left(r_{m}\right)}} \sum_{k=1}^{m} \frac{C_{1}^{F_{i_{k}}^{\left(r_{k}\right)}}}{\theta_{p}} \mathrm{e}^{-C_{2}^{F_{i_{k}}^{\left(r_{k}\right)}} n^{d-1}}\right\} \\
& \quad+M 3^{M^{2}} C_{d} n^{M(d-2)}\left(M c_{1} \mathrm{e}^{-c_{2} n^{1-3 / 2 d}}+D_{1} \mathrm{e}^{-D_{2} n^{(d-1) / 2 d}}\right) \\
& \leq b_{1} \mathrm{e}^{-b_{2} n^{1-3 / 2 d}}+b_{1}^{\prime} \mathrm{e}^{-b_{2}^{\prime} n} \\
& +C_{d} n^{M(d-2)} \sum_{m=1}^{M} 3^{M^{2}} \max _{r_{1}, \ldots, r_{m}}\left\{\sum_{i_{1}=1}^{N^{\left(r_{1}\right)}} \ldots \sum_{i_{m}=1}^{N^{\left(r_{m}\right)}} \sum_{k=1}^{m} \frac{C_{1}^{F_{i_{k}}^{\left(r_{k}\right)}}}{\theta_{p}} \mathrm{e}^{-C_{2}^{F_{i_{k}}} n^{\left(r_{k}\right)} n^{d-1}}\right\} \\
& +M 3^{M^{2}} C_{d} n^{M(d-2)}\left(M c_{1} \mathrm{e}^{-c_{2} n^{1-3 / 2 d}}+D_{1} \mathrm{e}^{-D_{2} n^{(d-1) / 2 d}}\right)
\end{aligned}
$$

where $C_{d}$ is a constant depending only on the dimension and the maximum is over $r_{1}, \ldots, r_{m} \in\left\{1, \ldots, 3^{M}\right\}$. We recall that $M, N$ and the number of ways of choosing $r_{1}, \ldots, r_{m}$ are finite and independent of $n$.

Remark 6.2. To obtain inequality (6.34), it is crucial to use a covering of $\mathcal{C}_{\beta}$ that is uniform in $y_{1}, \ldots, y_{m}$.

Let us assume $\mu_{n} \notin \mathcal{V}\left(\mathcal{W}, \mathfrak{F}_{n}, 2 \varepsilon\right)$. Let $\nu \in \mathcal{W}_{\xi}$, we can write $\nu=\nu_{x+r} W_{p}$ with $x \in \mathbb{R}^{d}$ and $r \in[1-\lambda, 1+2 \xi]$. We have for all $f \in \mathfrak{F}_{n}$

$$
\begin{aligned}
\left|\nu_{x+W_{p}}(f)-\nu_{x+r W_{p}}(f)\right| & \leq \max \left(\mathcal{L}^{d}\left(W_{p} \backslash(1-\lambda) W_{p}\right), \mathcal{L}^{d}\left((1+2 \xi) W_{p} \backslash W_{p}\right)\right) \\
& \leq c(p, d, \xi)
\end{aligned}
$$

where $c(p, d, \xi)$ is a constant that goes to 0 when $\xi$ goes to 0 . So that

$$
\sup _{f \in \widetilde{F}_{n}}\left|\nu_{x+W_{p}}(f)-\nu_{x+r W_{p}}(f)\right| \leq c(p, d, \xi) .
$$

As $\mu_{n} \notin \mathcal{V}\left(\mathcal{W}, \mathfrak{F}_{n}, 2 \varepsilon\right)$, we have

$$
\sup _{f \in \widetilde{F}_{n}}\left|\mu_{n}(f)-\nu_{x+W_{p}}(f)\right|>2 \varepsilon .
$$

So that up to choosing a smaller $\xi$, we have

$$
c(p, d, \xi) \leq \varepsilon
$$

and so

$$
\begin{aligned}
\mathbb{P}\left(\exists G_{n} \in \mathcal{G}_{n}, \forall \nu\right. & \left.\in \mathcal{W}, \sup _{f \in \widetilde{F}_{n}}\left|\mu_{n}(f)-\nu_{x+W_{p}}(f)\right|>2 \varepsilon\right) \\
& \leq \mathbb{P}\left(\exists G_{n} \in \mathcal{G}_{n}, \forall \nu \in \mathcal{W}_{\xi}, \sup _{f \in \widetilde{F}_{n}}\left|\mu_{n}(f)-\nu_{x+W_{p}}(f)\right|>\varepsilon\right) .
\end{aligned}
$$

Finally, using (6.34), there exist positive constants $C_{1}$ and $C_{2}$ depending on $\varepsilon, u, p$ and $d$ such that for all $n \geq 1$,

$$
\mathbb{P}\left(\exists G_{n} \in \mathcal{G}_{n}, \mu_{n} \notin \mathcal{V}\left(\mathcal{W}, \mathfrak{F}_{n}, 2 \varepsilon\right) \mid 0 \in \mathcal{C}_{\infty}\right) \leq C_{1} \mathrm{e}^{-c_{2} n^{1-3 / 2 d}}
$$


and the result follows.

To conclude, let us sum up the order in which the constants are chosen. We first choose $\varepsilon>0$. Next, we choose $\xi$ small enough such that it satisfies both inequalities (6.24) and (6.36), and $\delta^{\prime}$ such that it satisfies inequality (6.23). Next, we choose $\delta$ such that $\eta(\delta)$ satisfies inequalities (6.6), (6.20) and (6.22). We choose $\delta_{2}$ depending on $w$ (and so on $\varepsilon$ ) and $G$. The parameter $\delta_{2}$ has to satisfy some inequalities that we do not detail here, we refer to section 7 in Cerf and Théret (2011). Finally, to each $r$ in $\left\{1, \ldots, 3^{M}\right\}$, to each $F \in \mathcal{C}_{\beta}^{(r)}$, we choose $\varepsilon_{F}$ in such a way it satifies inequality (6.27).

6.2. Proof of Theorem 1.5. In this section we prove Theorem 1.5. Thanks to Theorem 1.7, we know that with high probability $\mu_{n}$ is close to the set $\mathcal{W}$ and so it is close to the measure of a translate of the Wulff shape. In fact, as $\mu_{n}$ has its support included in $B\left(0, n^{d-1}\right)$, the measure $\mu_{n}$ is close to $\mathcal{W}_{n}$, the set of measures defined as:

$$
\mathcal{W}_{n}=\left\{\nu_{x+W_{p}}, x \in B\left(0, n^{d-1}\right)\right\} .
$$

The continuous set $\mathcal{W}_{n}$ can be approximated by a finite set $\widetilde{\mathcal{W}}$ containing a polynomial number of measures such that $\mu_{n}$ is close to $\widetilde{\mathcal{W}}$ and so is close to at least one measure in $\widetilde{\mathcal{W}}$. Let $\varepsilon>0$ and let $w>0$ be a real number depending on $\varepsilon$ that we will choose later. We first use Lemma 6.1 , to cover $W_{p}$ by a finite number of balls of small radius such that $W_{p}$ is almost flat in each ball. Let $\delta_{2}$ that will be chosen later and let $\left(B\left(x_{k}, \rho_{k}, v_{k}\right)\right)_{k \in J}$ be a family associated to $W_{p}, \delta_{2}, \varepsilon$ that satisfies the conditions stated in Lemma 6.1. We will use this covering for all the translates of the Wulff shape. We set $\varepsilon_{W}=\min _{k \in J} \alpha_{d} \rho_{k}^{d} \delta_{2}$. We now cover $\mathcal{W}_{n}$ by a polynomial in $n$ number of balls of radius less than $\varepsilon_{W}$. Let $\xi>0$ small enough such that

$$
\forall x, y \in \mathbb{R}^{d},\|x-y\|_{2} \leq \xi \Longrightarrow \mathcal{L}^{d}\left(\left(x+W_{p}\right) \Delta\left(y+W_{p}\right)\right) \leq \frac{\varepsilon_{W}}{4} .
$$

By construction, $\mu_{n}$ has its support included in $B\left(0, n^{d-1}\right)$. We can cover $B\left(0, n^{d-1}\right)$ by a polynomial in $n$ number of balls of radius $\xi$. More precisely, there exist $z_{1}, \ldots, z_{M^{\prime}} \in B\left(0, n^{d-1}\right)$, such that $M^{\prime}$ is polynomial in $n$ and

We set

$$
B\left(0, n^{d-1}\right) \subset \bigcup_{i=1}^{M^{\prime}} B\left(z_{i}, \xi\right) .
$$

$$
\widetilde{\mathcal{W}}=\left\{\nu_{z_{i}+W_{p}}, i=1, \ldots, M^{\prime}\right\}
$$

Let $\delta>0$ we will choose later. We define $W_{p}^{\delta}$ and $W_{p}^{-\delta}$ as

$$
W_{p}^{\delta}=\left\{x \in \mathbb{R}^{d}: d_{2}\left(x, W_{p}\right) \leq \delta\right\} \text { and } W_{p}^{-\delta}=\left\{x \in W_{p}: d_{2}\left(x, \partial W_{p}\right) \geq \delta\right\} .
$$

Let us define $g$ as

$$
g(x)=\left\{\begin{array}{ll}
\min \left(d_{2}\left(x, W_{p}\right) / \delta, 1\right) & \text { if } x \in \mathbb{R}^{d} \backslash W_{p} \\
-\min \left(d_{2}\left(x, \partial W_{p}\right) / \delta, 1\right) & \text { if } x \in W_{p}
\end{array} .\right.
$$

The function $g$ is uniformly continuous and satisfies $\|g\|_{\infty} \leq 1$. For each $i \in$ $\left\{1, \ldots, M^{\prime}\right\}$, we define $g_{i}$ by $g_{i}(x)=g\left(x-z_{i}\right)$ for $x \in \mathbb{R}^{d}$, and $\mathfrak{F}=\left\{g_{i}, 1 \leq i \leq\right.$ $\left.M^{\prime}\right\} \cup\{1\}$. The set $\mathfrak{F}$ is a set made of translates of $g$ and the constant function 
equal to 1 . If the measure $\mu_{n}$ is in the local weak neighborhood $\mathcal{V}\left(\mathcal{W}, \mathfrak{F}, \frac{\varepsilon_{W}}{4}\right)$, then there exists $\nu_{x+W_{p}}$ in $\mathcal{V}\left(\mathcal{W}_{n}, \mathfrak{F}, \frac{\varepsilon_{W}}{4}\right)$ such that

$$
\sup _{f \in \mathfrak{F}}\left|\nu_{x+W_{p}}(f)-\mu_{n}(f)\right| \leq \frac{\varepsilon_{W}}{4} .
$$

Moreover there exists an $i \in\left\{1, \ldots, M^{\prime}\right\}$ such that $x \in B\left(z_{i}, \xi\right)$ and so

$$
\sup _{f \in \mathfrak{F}}\left|\nu_{x+W_{p}}(f)-\nu_{z_{i}+W_{p}}(f)\right| \leq \mathcal{L}^{d}\left(\left(x+W_{p}\right) \Delta\left(z_{i}+W_{p}\right)\right) \leq \frac{\varepsilon_{W}}{4}
$$

and also

$$
\mu_{n} \in \mathcal{V}\left(\widetilde{\mathcal{W}}, \mathfrak{F}, \varepsilon_{W} / 2\right)
$$

Let us choose $r>0$ large enough so that the ball $B(0, r-2 d)$ contains $W_{p}$. For $x \in \mathbb{R}^{d}$, we define $\lfloor x\rfloor$ to be the closest point to $x$ in $\mathbb{Z}^{d}$ for the Euclidean distance. For any $i \in\left\{1, \ldots, M^{\prime}\right\}$, we have

$$
W+z_{i} \subset B\left(\left\lfloor z_{i}\right\rfloor, r\right) .
$$

Let us define the function $u$ such that for all $\iota>0$,

$$
u(\iota)=\min \left(\sup \left\{\delta>0, \forall x, y \in \mathbb{R}^{d},\|x-y\|_{2} \leq \delta \Longrightarrow|g(x)-g(y)| \leq \iota\right\}, 1\right) .
$$

As the function $g$ is uniformly continuous, the function $u$ is positive. Moreover, as $\mathfrak{F}$ is made of translated of $g$ and the constant function equal to 1 , it is clear that this set satisfies the condition stated in Proposition 5.10 associated with the function $u$. Using Proposition 5.10 with the function $u$, there exist positive constants $C_{1}$, $C_{2}$ depending only on $r, u, p$ and $\varepsilon_{W}$ such that for all $i \in\left\{1, \ldots, M^{\prime}\right\}$

$$
\mathbb{P}\left(\max _{G_{n} \in \mathcal{G}_{n}} \sup _{f \in \mathfrak{F}}\left|\mu_{n}\left(f \mathbb{1}_{B\left(\left\lfloor z_{i}\right\rfloor, r\right)}\right)-\nu_{n}\left(f \mathbb{1}_{B\left(\left\lfloor z_{i}\right\rfloor, r\right)}\right)\right|>\varepsilon_{W} / 4\right) \leq C_{1} \mathrm{e}^{-c_{2} n^{1-3 / 2 d}} .
$$

The point of choosing such a set $\mathfrak{F}$ is that we can deduce from the fact that the quantity $\sup _{f \in \mathfrak{F}}\left|\mu_{n}(f)-\nu_{W+z_{i}}(f)\right|$ is small that the associated symmetric difference $\mathcal{L}^{d}\left(\left(P_{n} \cap B\left(\left\lfloor z_{i}\right\rfloor, r\right)\right) \Delta\left(z_{i}+W_{p}\right)\right)$ is small. Indeed, we have

$$
\begin{aligned}
\mathcal{L}^{d}( & \left.\left(P_{n} \cap B\left(\left\lfloor z_{i}\right\rfloor, r\right)\right) \Delta\left(z_{i}+W_{p}\right)\right) \\
= & \int_{\left(P_{n} \cap B\left(\left\lfloor z_{i}\right\rfloor, r\right)\right) \backslash\left(z_{i}+W_{p}\right)} 1 d \mathcal{L}^{d}(x)+\int_{\left(z_{i}+W_{p}\right) \backslash P_{n}} 1 d \mathcal{L}^{d}(x) \\
\leq & \int_{\left(P_{n} \cap B\left(\left\lfloor z_{i}\right\rfloor, r\right)\right) \backslash\left(z_{i}+W_{p}\right)} g_{i}(x) d \mathcal{L}^{d}(x)-\int_{\left(z_{i}+W_{p}\right) \backslash P_{n}} g_{i}(x) d \mathcal{L}^{d}(x) \\
& +\mathcal{L}^{d}\left(W_{p}^{\delta} \backslash W_{p}^{-\delta}\right) \\
= & \left|\nu_{n}\left(g_{i} \mathbb{1}_{\left.B\left(\left\lfloor z_{i}\right\rfloor, r\right)\right)}\right)-\nu_{W+z_{i}}\left(g_{i} \mathbb{1}_{\left.B\left(\left\lfloor z_{i}\right\rfloor, r\right)\right)}\right)\right|+\mathcal{L}^{d}\left(W_{p}^{\delta} \backslash W_{p}^{-\delta}\right) \\
\leq & \sup _{f \in \mathfrak{F}} \mid \mu_{n}\left(f \mathbb{1}_{B\left(\left\lfloor z_{i}\right\rfloor, r\right)}\right)-\nu_{n}\left(f \mathbb{1}_{B\left(\left\lfloor z_{i}\right\rfloor, r\right)} \mid\right. \\
& \quad+\sup _{f \in \mathfrak{F}}\left|\mu_{n}\left(f \mathbb{1}_{B\left(\left\lfloor z_{i}\right\rfloor, r\right)}\right)-\nu_{W+z_{i}}\left(f \mathbb{1}_{B\left(\left\lfloor z_{i}\right\rfloor, r\right)}\right)\right|+\mathcal{L}^{d}\left(W_{p}^{\delta} \backslash W_{p}^{-\delta}\right) .
\end{aligned}
$$

So we choose $\delta$ small enough so that

$$
\mathcal{L}^{d}\left(W_{p}^{\delta} \backslash W_{p}^{-\delta}\right) \leq \frac{\varepsilon_{W}}{4} .
$$


Moreover, we have

$$
\begin{aligned}
\underline{\mathbb{P}}\left(\exists G_{n}\right. & \left.\in \mathcal{G}_{n},\left|\partial^{o} G_{n}\right| \leq(1-w) \mathcal{I}_{p}\left(W_{p}\right) n^{d-1}, \mu_{n} \in \mathcal{V}\left(\widetilde{\mathcal{W}}, \mathfrak{F}, \varepsilon_{W} / 2\right)\right) \\
& \leq \sum_{i=1}^{M^{\prime}} \mathbb{P}\left(\begin{array}{c}
\exists G_{n} \in \mathcal{G}_{n},\left|\partial^{o} G_{n}\right| \leq(1-w) \mathcal{I}_{p}\left(W_{p}\right) n^{d-1} \\
\sup _{f \in \mathfrak{F}}\left|\mu_{n}(f)-\nu_{W+z_{i}}(f)\right| \leq \varepsilon_{W} / 2
\end{array} \mid 0 \in \mathcal{C}_{\infty}\right) .
\end{aligned}
$$

Using inequalities (6.37), (6.38) and (6.39), we obtain

$$
\begin{aligned}
& \mathbb{P}\left(\exists G_{n} \in \mathcal{G}_{n}, \quad \sup _{f \in \mathfrak{F}}\left|\mu_{n}(f)-\nu_{W+z_{i}}(f)\right| \leq \varepsilon_{W} / 2 \mid 0 \in \mathcal{C}_{\infty}\right) \\
& \leq \underline{\mathbb{P}}\left(\exists G_{n} \in \mathcal{G}_{n}, \quad \begin{array}{c}
\left|\partial^{o} G_{n}\right| \leq(1-w) \mathcal{I}_{p}\left(W_{p}\right) n^{d-1}, \\
\sup _{f \in \mathfrak{F}}\left|\mu_{n}\left(f \mathbb{1}_{B\left(\left\lfloor z_{i}\right\rfloor, r\right)}\right)-\nu_{W+z_{i}}\left(f \mathbb{1}_{B\left(\left\lfloor z_{i}\right\rfloor, r\right)}\right)\right| \leq \varepsilon_{W} / 2
\end{array}\right)
\end{aligned}
$$



Finally, we proceed as in inequality (6.32) in the proof of Theorem 1.7:

$$
\begin{aligned}
\mathbb{P}\left(\exists G_{n} \in \mathcal{G}_{n}, \quad \partial^{o} G_{n} \mid\right. & \leq(1-w) \mathcal{I}_{p}\left(W_{p}\right) n^{d-1}, \\
\mathcal{L}^{d}\left(\left(P_{n} \cap B(\right.\right. & \left.\left.\left.\left(\left\lfloor z_{i}\right\rfloor, r\right)\right) \Delta\left(z_{i}+W_{p}\right)\right) \leq \varepsilon_{W} \mid 0 \in \mathcal{C}_{\infty}\right) \\
& \leq \frac{1}{\theta_{p}} \sum_{k \in J} \mathbb{P}\left(G\left(z_{i}+x_{k}, \rho_{k}, n_{W_{p}}\left(x_{k}\right), w, \delta_{2}\right)\right)
\end{aligned}
$$

It is possible to choose $\delta_{2}$ depending on $W, G$ and $w$ (see again section 6 in Cerf and Théret, 2011) such that there exist positive constants $C_{1, k}$ and $C_{2, k}$ depending on $G, d, W, k$ and $w$ so that for all $k \in J$,

$$
\mathbb{P}\left[G\left(x_{k}, \rho_{k}, n_{W_{p}}\left(x_{k}\right), w, \delta_{2}\right)\right] \leq C_{1, k} \exp \left(-C_{2, k} n^{d-1}\right) .
$$

So combining inequalities (6.40), (6.41) and (6.42), we obtain

$$
\begin{aligned}
\underline{\mathbb{P}}\left(\exists G_{n} \in \mathcal{G}_{n},\left|\partial^{o} G_{n}\right|\right. & \left.\leq(1-w) \mathcal{I}_{p}\left(W_{p}\right) n^{d-1}, \mu_{n} \in \mathcal{V}\left(\widetilde{\mathcal{W}}, \mathfrak{F}, \varepsilon_{W} / 2\right)\right) \\
\leq & M^{\prime}\left(C_{1} \mathrm{e}^{-c_{2} n^{1-3 / 2 d}}+\frac{1}{\theta_{p}} \sum_{k \in J} C_{1, k} \exp \left(-C_{2, k} n^{d-1}\right)\right) .
\end{aligned}
$$

Moreover, we have

$$
\begin{aligned}
\underline{\mathbb{P}}\left(\exists G_{n} \in \mathcal{G}_{n},\right. & \left.\frac{\left|G_{n}\right|}{n^{d}} \geq(1+w) \theta_{p} \mathcal{L}^{d}\left(W_{p}\right), \mu_{n} \in \mathcal{V}\left(\widetilde{\mathcal{W}}, \mathfrak{F}, \varepsilon_{W} / 2\right)\right) \\
& \leq \sum_{i=1}^{M^{\prime}} \mathbb{P}\left(\begin{array}{c}
\left.\exists G_{n} \in \mathcal{G}_{n}, \frac{\left|G_{n}\right|}{n^{d}} \geq(1+w) \theta_{p} \mathcal{L}^{d}\left(W_{p}\right), \quad \mid 0 \in \mathcal{C}_{\infty}\right) \\
\left|\mu_{n}(1)-\nu_{W+z_{i}}(1)\right| \leq \varepsilon_{W} / 2
\end{array}\right) \\
& \leq \sum_{i=1}^{M^{\prime}} \mathbb{P}\left(\begin{array}{c}
\exists G_{n} \in \mathcal{G}_{n}, \frac{\left|G_{n}\right|}{n^{d}} \geq(1+w) \theta_{p} \mathcal{L}^{d}\left(W_{p}\right), \quad \mid 0 \in \mathcal{C}_{\infty} \\
\left|\frac{\left|G_{n}\right|}{n^{d}}-\theta_{p} \mathcal{L}^{d}\left(W_{p}\right)\right| \leq \varepsilon_{W} / 2
\end{array}\right)
\end{aligned}
$$

where we recall that $\theta_{p} \mathcal{L}^{d}\left(W_{p}\right)=1$, so up to choosing a smaller $\varepsilon_{W}$, we assume that $\varepsilon_{W} \leq 2 w$ so that the probability in the sum is equal to 0 . Finally, combining inequalities (6.43) and (6.44), we obtain

$$
\mathbb{P}\left(n \varphi_{n} \geq \frac{1-w}{1+w} \frac{\mathcal{I}_{p}\left(W_{p}\right)}{\theta_{p} \mathcal{L}^{d}\left(W_{p}\right)} \mid 0 \in \mathcal{C}_{\infty}\right) \leq \underline{\mathbb{P}}\left(\exists G_{n} \in \mathcal{G}_{n}, \mu_{n} \notin \mathcal{V}\left(\widetilde{\mathcal{W}}, \mathfrak{F}, \varepsilon_{W} / 2\right)\right)
$$




$$
+M^{\prime}\left(C_{1} \exp \left(-C_{2} n\right)+\frac{1}{\theta_{p}} \sum_{k \in J} C_{1, k} \exp \left(-C_{2, k} n^{d-1}\right)\right) .
$$

Thanks to Theorem 1.7, there exist positive constants $C_{1}^{\prime}, C_{2}^{\prime}$, depending on $p, u$, $\varepsilon_{W}$ and $d$ such that

$$
\mathbb{P}\left(\exists G_{n} \in \mathcal{G}_{n}, \mu_{n} \notin \mathcal{V}\left(\widetilde{\mathcal{W}}, \mathfrak{F}, \varepsilon_{W} / 2\right) \mid 0 \in \mathcal{C}_{\infty}\right) \leq C_{1}^{\prime} \exp \left(-C_{2}^{\prime} n^{1-3 / 2 d}\right) .
$$

By choosing $w$ small enough, we obtain

$$
\begin{aligned}
& \mathbb{P}\left(n \varphi_{n} \geq(1-\varepsilon) \frac{\mathcal{I}_{p}\left(W_{p}\right)}{\theta_{p} \mathcal{L}^{d}\left(W_{p}\right)} \mid 0 \in \mathcal{C}_{\infty}\right) \\
& \quad \leq C_{1}^{\prime} \exp \left(-C_{2}^{\prime} n^{1-3 / 2 d}\right)+M^{\prime}\left(C_{1} \exp \left(-C_{2} n\right)+\sum_{k \in J} C_{1, k} \exp \left(-C_{2, k} n^{d-1}\right)\right) .
\end{aligned}
$$

As $M^{\prime}$ is polynomial in $n$, the result follows.

6.3. Proof of Theorem 1.2. Let $\varepsilon>0$. As in the proof of Theorem 1.5, there exists an integer $M^{\prime}$ that is polynomial in $n$ and $z_{1}, \ldots, z_{M^{\prime}}$ points of $B\left(0, n^{d-1}\right)$ such that for any finite set $\mathfrak{F}$ of continuous functions of infinite norm at most 1 , if $\mu_{n} \in \mathcal{V}(\mathcal{W}, \mathfrak{F}, \varepsilon)$ then $\mu_{n} \in \mathcal{V}(\widetilde{\mathcal{W}}, \mathfrak{F}, 2 \varepsilon)$ where $\widetilde{\mathcal{W}}=\left\{\nu_{z_{i}+W_{p}}, i=1, \ldots, M^{\prime}\right\}$. Let $\delta>0$ we will choose later. Let us define $f$ and $g$ as

$$
f(x)=\min \left(d_{2}\left(x, \mathbb{R}^{d} \backslash W_{p}^{\delta}\right) / \delta, 1\right), \text { for } x \in \mathbb{R}^{d}
$$

and

$$
g(x)=\min \left(d_{2}\left(x, W_{p}\right) / \delta, 1\right), \text { for } x \in \mathbb{R}^{d} .
$$

The functions $f$ and $g$ are uniformly continuous and satisfy $\|f\|_{\infty} \leq 1$ and $\|g\|_{\infty} \leq$ 1. For each $i \in\left\{1, \ldots, M^{\prime}\right\}$, we define $f_{i}$ by $f_{i}(x)=f\left(x-z_{i}\right)$ and $g_{i}$ by $g_{i}(x)=$ $f\left(x-z_{i}\right)$ for $x \in \mathbb{R}^{d}$. We define

$$
\mathfrak{F}=\left\{f_{i}, 1 \leq i \leq M^{\prime}\right\} \cup\left\{g_{i}, 1 \leq i \leq M^{\prime}\right\}
$$

Let $G_{n} \in \mathcal{G}_{n}$. Let $i \in\left\{1, \ldots, M^{\prime}\right\}$. We have

$$
\left|G_{n} \Delta\left(\left(n\left(W_{p}+z_{i}\right)\right) \cap \mathcal{C}_{\infty}\right)\right|=\left|G_{n} \backslash n\left(W_{p}+z_{i}\right)\right|+\left|\left(n\left(W_{p}+z_{i}\right) \cap \mathcal{C}_{\infty}\right) \backslash G_{n}\right|
$$

Using a renormalization argument as in the proof of Theorem 1.4, there exist positive constants $C_{1}$ and $C_{2}$ depending on $p, \varepsilon$ and $d$ such that for all $i \in\left\{1, \ldots, M^{\prime}\right\}$,

$$
\mathbb{P}\left(\left|\frac{\left|\left(n\left(W+z_{i}\right)\right) \cap \mathcal{C}_{\infty}\right|}{n^{d}}-\theta_{p} \mathcal{L}^{d}\left(W_{p}\right)\right| \geq \varepsilon \mid 0 \in \mathcal{C}_{\infty}\right) \leq C_{1} \exp \left(-C_{2} n\right) .
$$

As $G_{n} \cap\left(n\left(W_{p}+z_{i}\right)\right) \subset\left(n\left(W_{p}+z_{i}\right)\right) \cap \mathcal{C}_{\infty}$, we have with probability at least $1-C_{1} \exp \left(-C_{2} n\right)$,

$$
\begin{aligned}
& \mid((n) \\
& \left.\left.\quad=\mid\left(W_{p}+z_{i}\right)\right) \cap \mathcal{C}_{\infty}\right) \backslash G_{n} \mid \\
& \left.\quad \leq \theta_{p} \mathcal{L}^{d}\left(W_{p}\right)\right) \cap n^{d}+n^{d} \varepsilon-n^{d} \mu_{n}\left(f_{i}\right)+\left|n\left(\left(W_{p}^{\delta}+z_{i}\right) \backslash\left(W_{p}+z_{i}\right)\right) \cap \mathbb{Z}^{d}\right| .
\end{aligned}
$$

We can find a constant $c(\delta)$ depending only on $\delta, p$ and $d$, such that $c(\delta)$ goes to 0 when $\delta$ goes to 0 and for all $z \in \mathbb{R}^{d}$

$$
\left|n\left(\left(W_{p}^{\delta}+z\right) \backslash\left(W_{p}+z\right)\right) \cap \mathbb{Z}^{d}\right| \leq c(\delta) n^{d},
$$


so that,

$$
\begin{aligned}
\left|\left(\left(n\left(W_{p}+z_{i}\right)\right) \cap \mathcal{C}_{\infty}\right) \backslash G_{n}\right| & \leq n^{d}\left|\nu_{W_{p}+z_{i}}\left(f_{i}\right)-\mu_{n}\left(f_{i}\right)\right|+(\varepsilon+c(\delta)) n^{d} \\
& \leq n^{d} \sup _{h \in \mathfrak{F}}\left|\nu_{W_{p}+z_{i}}(h)-\mu_{n}(h)\right|+(\varepsilon+c(\delta)) n^{d} .
\end{aligned}
$$

Moreover, noticing that $\nu_{W_{p}+z_{i}}\left(g_{i}\right)=0$, we obtain

$$
\begin{aligned}
\left|G_{n} \backslash n\left(W+z_{i}\right)\right| & \leq n^{d} \mu_{n}\left(g_{i}\right)+\left|n\left(\left(W_{p}^{\delta}+z_{i}\right) \backslash\left(W_{p}+z_{i}\right)\right) \cap \mathbb{Z}^{d}\right| \\
& \leq n^{d}\left|\mu_{n}\left(g_{i}\right)-\nu_{W_{p}+z_{i}}\left(g_{i}\right)\right|+n^{d} c(\delta) \\
& \leq n^{d} \sup _{h \in \mathfrak{F}}\left|\nu_{W_{p}+z_{i}}(h)-\mu_{n}(h)\right|+n^{d} c(\delta) .
\end{aligned}
$$

Combining inequalities (6.46), (6.47) and (6.48), with high probability, we have

$$
\begin{aligned}
\inf _{z \in \mathbb{R}^{d}} \frac{1}{n^{d}}\left|G_{n} \Delta\left(\left(n\left(W_{p}+z\right)\right) \cap \mathcal{C}_{\infty}\right)\right| \\
\quad \leq \min _{1 \leq i \leq M^{\prime}} \frac{1}{n^{d}}\left|G_{n} \Delta\left(\left(n\left(W_{p}+z_{i}\right)\right) \cap \mathcal{C}_{\infty}\right)\right| \\
\quad \leq \min _{\nu \in \widetilde{\mathcal{W}}}\left\{\sup _{h \in \mathfrak{F}}\left|\nu(h)-\mu_{n}(h)\right|+\sup _{h \in \mathfrak{F}}\left|\nu(h)-\mu_{n}(h)\right|\right\}+\varepsilon+2 c(\delta) \\
\quad \leq 2 \min _{\nu \in \mathcal{W}} \sup _{h \in \mathfrak{F}}\left|\nu(h)-\mu_{n}(h)\right|+\varepsilon+2 c(\delta) .
\end{aligned}
$$

Let us define for any $\iota>0$,

$$
\begin{aligned}
& u_{g}(\iota)=\min \left(\sup \left\{\delta>0, \forall x, y \in \mathbb{R}^{d},\|x-y\|_{2} \leq \delta \Longrightarrow|g(x)-g(y)| \leq \iota\right\}, 1\right), \\
& u_{f}(\iota)=\min \left(\sup \left\{\delta>0, \forall x, y \in \mathbb{R}^{d},\|x-y\|_{2} \leq \delta \Longrightarrow|f(x)-f(y)| \leq \iota\right\}, 1\right)
\end{aligned}
$$

and $u=\min \left(u_{f}, u_{g}\right)$. This function is positive because the function $f$ and $g$ are uniformly continuous. It is easy to check that $\mathfrak{F}$ satisfies the condition required in Theorem 1.7 associated with the function $u$. Thus, there exist positive constants $c_{1}$ and $c_{2}$ depending on $p, u, \varepsilon$ and $d$ such that

$$
\mathbb{P}\left(\exists G_{n} \in \mathcal{G}_{n}, \inf _{\nu \in \mathcal{W}} \sup _{h \in \mathfrak{F}}\left|\nu(h)-\mu_{n}(h)\right| \geq \varepsilon \mid 0 \in \mathcal{C}_{\infty}\right) \leq c_{1} \mathrm{e}^{-c_{2} n^{1-3 / 2 d}}
$$

and so

$$
\mathbb{P}\left(\exists G_{n} \in \mathcal{G}_{n}, \min _{\nu \in \mathcal{W}} \sup _{h \in \mathfrak{F}}\left|\nu(h)-\mu_{n}(h)\right| \geq 2 \varepsilon \mid 0 \in \mathcal{C}_{\infty}\right) \leq c_{1} \mathrm{e}^{-c_{2} n^{1-3 / 2 d}} .
$$

We now choose $\delta$ small enough such that $c(\delta) \leq \varepsilon$ so that

$$
\begin{array}{r}
\mathbb{P}\left(\exists G_{n} \in \mathcal{G}_{n}, \inf _{z \in \mathbb{R}^{d}} \frac{1}{n^{d}}\left|G_{n} \Delta\left(\left(n\left(W_{p}+z\right)\right) \cap \mathcal{C}_{\infty}\right)\right| \geq 7 \varepsilon \mid 0 \in \mathcal{C}_{\infty}\right) \\
\leq c_{1} \mathrm{e}^{-c_{2} n^{1-3 / 2 d}}+M^{\prime} C_{1} \exp \left(-C_{2} n\right) .
\end{array}
$$

As $M^{\prime}$ is polynomial in $n$, this yields the result.

\section{Acknowledgements}

I wish to express my gratitude to Raphaël Cerf for showing me this problem and for giving me the opportunity to work with him for an internship. I thank him for our fruitful discussions and his patience. This research was partially supported by the ANR project PPPP (ANR-16-CE40-0016). 


\section{References}

Alon, N. and Milman, V. D. $\lambda_{1}$, isoperimetric inequalities for graphs, and superconcentrators. J. Combin. Theory Ser. B, 38 (1), 73-88 (1985). MR782626.

Benjamini, I. and Mossel, E. On the mixing time of a simple random walk on the super critical percolation cluster. Probab. Theory Related Fields, 125 (3), 408-420 (2003). MR1967022.

Berger, N., Biskup, M., Hoffman, C. E., and Kozma, G. Anomalous heat-kernel decay for random walk among bounded random conductances. Ann. Inst. Henri Poincaré Probab. Stat., 44 (2), 374-392 (2008). MR2446329.

Biskup, M., Louidor, O., Procaccia, E. B., and Rosenthal, R. Isoperimetry in twodimensional percolation. Comm. Pure Appl. Math., 68 (9), 1483-1531 (2015). MR3378192.

Bollobás, B. Graph theory. An introductory course, volume 63 of Graduate Texts in Mathematics. Springer-Verlag, New York-Berlin (1979). ISBN 0-387-90399-2. MR536131.

Cerf, R. The Wulff crystal in Ising and percolation models, volume 1878 of Lecture Notes in Mathematics. Springer-Verlag, Berlin (2006). ISBN 978-3-540-30988-8; 3-540-30988-8. MR2241754.

Cerf, R. and Dembin, B. Vanishing of the anchored isoperimetric profile in bond percolation at $p_{c}$. Electron. Commun. Probab., 25, 7 pages, paper $n^{\circ} 2$ (2020). DOI: $10.1214 / 19-E C P 281$.

Cerf, R. and Pisztora, A. On the Wulff crystal in the Ising model. Ann. Probab., 28 (3), 947-1017 (2000). MR1797302.

Cerf, R. and Théret, M. Lower large deviations for the maximal flow through a domain of $\mathbb{R}^{d}$ in first passage percolation. Probab. Theory Related Fields, 150 (34), 635-661 (2011). MR2824869.

Cheeger, J. A lower bound for the smallest eigenvalue of the Laplacian. In Problems in analysis (Papers dedicated to Salomon Bochner, 1969), pp. 195-199 (1970). MR0402831.

Fabien Rau, C. Marches aléatoires sur un amas de percolation. Ph.D. thesis, Mathématiques appliquées Aix-Marseille (2006). http://www.theses.fr/ 2006 AIX11045.

Gabber, O. and Galil, Z. Explicit constructions of linear-sized superconcentrators. J. Comput. System Sci., 22 (3), 407-420 (1981). MR633542.

Giusti, E. Minimal surfaces and functions of bounded variation, volume 80 of Monographs in Mathematics. Birkhäuser Verlag, Basel (1984). ISBN 0-81763153-4. MR775682.

Gold, J. Isoperimetry in supercritical bond percolation in dimensions three and higher. Ann. Inst. Henri Poincaré Probab. Stat., 54 (4), 2092-2158 (2018). MR3865668.

Grimmett, G. Percolation, volume 321 of Grundlehren der Mathematischen Wissenschaften [Fundamental Principles of Mathematical Sciences]. Springer-Verlag, Berlin, second edition (1999). ISBN 3-540-64902-6. MR1707339.

Kesten, H. Aspects of first passage percolation. In École d'été de probabilités de Saint-Flour, XIV-1984, volume 1180 of Lecture Notes in Math., pp. 125-264. Springer, Berlin (1986). MR876084.

Liggett, T. M., Schonmann, R. H., and Stacey, A. M. Domination by product measures. Ann. Probab., 25 (1), 71-95 (1997). MR1428500. 
Mathieu, P. and Remy, E. Isoperimetry and heat kernel decay on percolation clusters. Ann. Probab., 32 (1A), 100-128 (2004). MR2040777.

Pete, G. A note on percolation on $\mathbb{Z}^{d}$ : isoperimetric profile via exponential cluster repulsion. Electron. Commun. Probab., 13, 377-392 (2008). MR2415145.

Pisztora, A. Surface order large deviations for Ising, Potts and percolation models. Probab. Theory Related Fields, 104 (4), 427-466 (1996). MR1384040.

Rossignol, R. and Théret, M. Lower large deviations and laws of large numbers for maximal flows through a box in first passage percolation. Ann. Inst. Henri Poincaré Probab. Stat., 46 (4), 1093-1131 (2010). MR2744888.

Rossignol, R. and Théret, M. Existence and continuity of the flow constant in first passage percolation. Electron. J. Probab., 23, Paper No. 99, 42 (2018). MR3862614.

Taylor, J. E. Unique structure of solutions to a class of nonelliptic variational problems. In Differential geometry (Proc. Sympos. Pure. Math., Vol. XXVII, Stanford Univ., Stanford, Calif., 1973), Part 1, pp. 419-427 (1975). MR0388225.

Theret, M. Upper large deviations for maximal flows through a tilted cylinder. ESAIM Probab. Stat., 18, 117-129 (2014). MR3143735.

Wulff, G. Zur Frage der Geschwindigkeit des Wachsthums und der Auflösung der Krystallflächen, volume 34 (1901). ISBN 21967105. DOI: 10.1524/zkri.1901.34.1.449.

Zhang, Y. Limit theorems for maximum flows on a lattice. Probab. Theory Related Fields, 171 (1-2), 149-202 (2018). MR3800832. 